\title{
Energy Prices and Discount Factors for Life-Cycle Cost Analysis 1994
}

Annual Supplement to

NIST Handbook 135 and

NBS Special Publication 709
Stephen R. Petersen

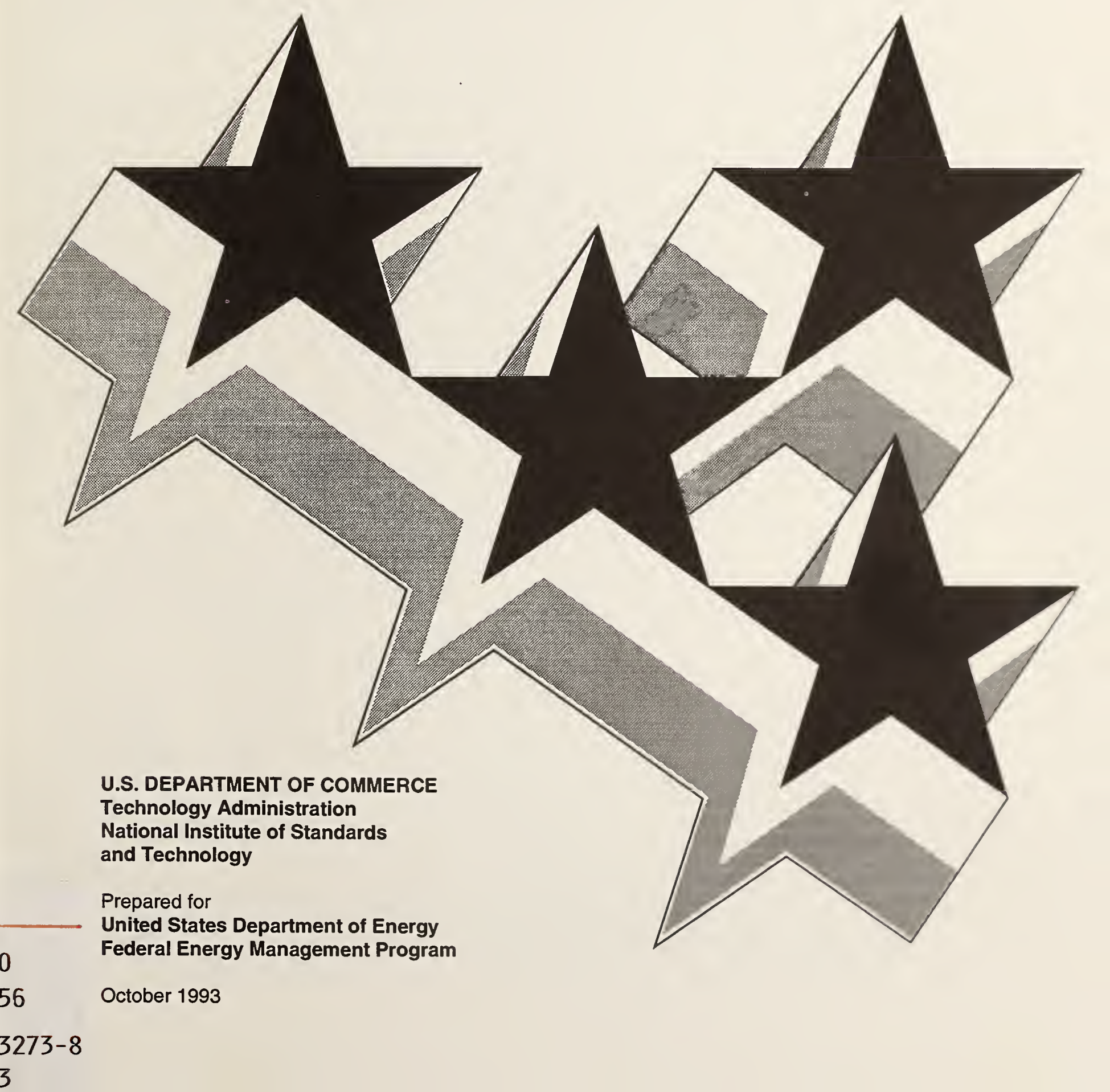





\section{ENERGY PRICES AND DISCOUNT FACTORS FOR LIFE-CYCLE COST ANALYSIS 1994}

Annual Supplement to

NIST Handbook 135 and

NBS Special Publication 709

Data for the Federal Methodology for Life-Cycle Cost Analysis, Title 10, CFR, Part 436, Subpart A; and for the Energy Conservation Mandatory Performance Standards for New

Federal Residential Buildings, Tiitle 10, CFR, Part 435

Stephen R. Petersen

Computing and Applied Mathematics Laboratory Office of Applied Economics Gaithersburg, MD 20899

October 1993

Prepared for:

U.S. Department of Energy

Office of the Assistant Secretary for Conservation and Renewable Energy Federal Energy Management Program Washington, DC 20585

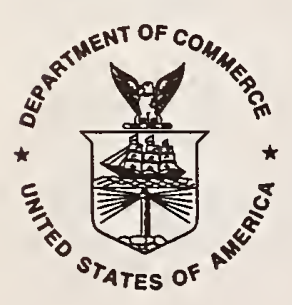





\section{FOREWORD}

Please note that Federal Methodology for Life-Cycle Cost Analysis, Title 10, CFR, Part 436, Subpart A was revised in November 1990 to incorporate changes required by the Federal Energy Management Improvement Act of 1988 (P.L. 100-615), and to reflect 10-years experience with the Federal LCC Rule.

The principal change resulted in the FEMP discount rate being set annually by DOE. The FEMP discount rate for 1994 is $3.1 \%$ real (i.e., net of general price inflation), equivalent to a market rate of $7.0 \%$. The FEMP discount rate is based on long-term Treasury bond rates averaged over the previous 12 months. This market rate is converted to a real rate using the assumed rate of general price inflation in the Economic Report of the President's Council of Economic Advisors, to correspond with the constant-dollar analysis approach that is used. The results are identical to those that would be obtained by using the $7.0 \%$ market rate as the discount rate and inflating all cash flows at the assumed rate of inflation. (For further discussion of changes in the Federal life-cycle costing rule see Notice of Final Rulemaking, Federal Register, November 20, 1990, Vol. 55, No. 224.)

In addition, for the first time this Annual Supplement uses the term "present value" instead of "present worth" for the discount factors presented. The meaning of these two terms is considered to be identical for purposes of economic analysis. This change in terminology was made to be consistent with the terms used in the American Society for Testing and Materials (ASTM) compilation of standards on building economics (ASTM Standards on Building Economics, Second Edition, ASTM, Philadelphia, PA, 1992.)

The SPV, UPV, and UPV* factors in Part I of this report are given both for the FEMP discount rate and for the OMB Circular A-94 discount rates. The former are for evaluating Federal energy conservation and renewable energy projects. The latter are for evaluating Federal projects subject to OMB Circular A-94, i.e., most Federal capital investment projects, other than energy projects and water-resources projects.

It should be noted that OMB Circular A-94 on guidelines and discount rates for benefit-cost analysis of federal programs was extensively revised in October 1992. There is no longer a single OMB discount rate as in the past. OMB has specified two basic types of discount rates: (1) a discount rate for cost-effectiveness, lease-purchase, and related analyses; and (2) a discount rate for public investment and regulatory analyses. Only discount rates for the first type of analyses are included in this Annual Supplement, since the primary purpose of this report is to support cost-effectiveness studies related to the design and operation of federal facilities.

Discount rates for cost-effectiveness and lease-purchase studies are based on interest rates on Treasury Notes and Bonds with maturities ranging from 3 to 30 years. Currently (as of February 1993) five maturities have been specifically identified by OMB, and are shown here with the corresponding real interest rate to be used as the discount rate for studies subject to OMB Circular A-94:

$\begin{array}{cccccc}\text { Maturity: } & \frac{3 \text {-year }}{3.1 \%} & \frac{5 \text {-year }}{3.6 \%} & \frac{7 \text {-year }}{4.0 \%} & \frac{10 \text {-year }}{4.3 \%} & \frac{30 \text {-year }}{4.5 \%}\end{array}$


OMB suggests that the actual discount rate for an economic analysis be interpolated from these maturities and rates, based on the study period used in the analysis.

Due to limitations on the size of this Annual Supplement, discount factors for only two of these maturities are presented: a rate for short-term analyses (up to 10 years) based on the 7-year rate (4.0\%), and a rate for long-term analyses (longer than 10 years) based on the 30-year rate $(4.5 \%)$. As a result, these discount factors are for approximation purposes only. It is suggested that the NIST Building Life Cycle Cost (BLCC) or DISCOUNT programs be used to compute the present value factors for the discount rate corresponding to the length of the study period when approximate values are not satisfactory for the project analysis. 


\section{PREFACE}

This is the 1994 edition of energy prices and discount factors for life-cycle cost analysis as established by the U.S. Department of Energy (DOE) in Subpart A of Part 436 of Title 10 of the Code of Federal Regulations (10 CFR Part 436, Subpart A), and amplified in the Life-Cycle Costing Manual for the Federal Energy Management Program (NIST Handbook 135). The data are provided as an aid to implementing life-cycle cost evaluations of potential energy conservation and renewable energy investments in existing and new Federally owned and leased buildings.

The life-cycle costing methods and procedures as set forth in 10 CFR, Part 436, Subpart A, are to be followed by all Federal agencies, unless specifically exempted, in evaluating the cost effectiveness of potential energy conservation and renewable energy investments in Federally owned and leased buildings.

As called for by legislation, the National Institute of Standards and Technology has provided technical assistance to the U.S. Department of Energy in the development and implementation of lifecycle costing methods and procedures. This is the second of a three-volume set which together provide the methods, data, and computational tools for life-cycle cost analysis of Federal energy projects.

Included in the three-volume set for Federal life-cycle cost analysis are the following:

(1) Life-Cycle Costing Manual for the Federal Energy Management Program, National Institute of Standards and Technology, Handbook 135 (1987). (Under Revision)

The manual is a guide to understanding life-cycle costing and related methods of economic analysis as they are applied to Federal decisions. It describes the required procedures and assumptions, defines and explains how to apply and interpret economic performance measures, gives examples of Federal decision problems and their solutions, explains how to use the energy price indices and discount factors which are updated annually in the supplement, and provides worksheets and other computational aids and instructions for calculating the required measures.

(2) Energy Prices and Discount Factors for Life-Cycle Cost Analysis, National Institute of Standards and Technology, NISTIR 85-3273 (updated annually).

This report, which is updated annually, gives the energy price and discount factor multipliers needed to estimate the present value of energy and other future costs. The data are based on energy price projections developed by the Energy Information Administration of the U.S. Department of Energy. Request the latest edition when ordering.

(3) NIST "Building Life Cycle Cost" (BLCC) Computer Program (version 4.1), National Institute of Standards and Technology, NISTIR 4481 (May 1993). 
The NIST BLCC program (version 4.1) supersedes and incorporates both the Federal Buildings LifeCycle Cost (FBLCC) and National Bureau of Standards Life-Cycle Cost (NBSLCC) programs. NIST BLCC is designed to run on IBM PC and compatible microcomputers with approximately $512 \mathrm{~K}$ of random access memory (RAM). It can be used to calculate the LCC of capital investments in buildings and building systems which are intended to reduce future operating, maintenance, and energy costs. BLCC computes the LCC for each alternative, compares alternatives in order to determine which has the lowest LCC, performs cash flow analyses, and then computes the net savings, savings-to-investment ratio (SIR), and adjusted internal rate of return (AIRR) over the designated study period. BLCC can be used to perform economic analysis of Federal and of private sector projects. BLCC (version 4.1) uses the 1994 energy price data in NISTIR 85-3273-8. BLCC in its application to Federal energy conservation and renewable energy projects is consistent with NIST Handbook 135 (see \#1 above). In its application to non-energy projects, BLCC is consistent with OMB Circular A-94. In its application to private-sector and non-Federal public-sector projects, BLCC is consistent with ASTM standards for building economics. BLCC is integrated with the DOE ASEAM computer program which performs energy conservation analysis.

Included on the BLCC disk is a stand-alone program called DISCOUNT (version 3.3) which can calculate present value, future value, and annual value factors for any discount rate and study period. DISCOUNT can access the DOE energy price projections included on the BLCC disk to compute the UPV* factors needed for Federal LCC analyses of energy projects, consistent with the factors included in this report.

The three-volume set can also be used to perform economic evaluations of Federal building projects which are not primarily for conserving energy or providing renewable energy but which have an energy cost component. NIST Handbook 135 explains both applications.

The U.S. Department of Energy was directed by legislation and executive order to make available to the private sector the methods, procedures, and related aids developed for Federal use. In response to this directive, the National Institute of Standards and Technology, under sponsorship of the U.S. Department of Energy, published a life-cycle costing book for use by the private sector entitled Comprehensive Guide for Least-Cost Energy Decisions, NBS SP 709 (January 1987). The private sector guide is supported by the data provided here, as well as by the BLCC computer program. The BLCC program (version 4.1) supersedes the NBSLCC program which is documented in SP 709. BLCC provides LCC computational support for private sector projects as well as for Federal projects.

To order any of the printed publications contact:

Advanced Sciences, Inc.

2000 North 15th Street

Suite 407

Arlington, VA 22201

Telephone (703) 243-4900

Please request the publications by name and number. 
To order BLCC for analyses of Federal buildings, contact the above address. Other users may order BLCC from one of the following:

\author{
PC Software Interest Group \\ 1030D E. Duane Ave. \\ Sunnyvale, CA 94086 \\ (408) $730-9291$ \\ MTS Software \\ 5 Oak Court \\ Saint Charles, MO 63302 \\ (314) 441-1022 \\ Energy Information Services \\ P.O. Box 381 \\ St. Johnsbury, VT 05819-0381 \\ (802) $748-5148$
}

Workshops on the life-cycle costing method and energy analysis are conducted at locations around the country each year. The workshops include training and software for both BLCC and an energy analysis computer program called "A Simplified Energy Analysis Method" (ASEAM). A schedule of workshops can be obtained from the Office of Applied Economics, National Institute of Standards and Technology, Building 226, Room B226, Gaithersburg, MD 20899, Telephone (301) 975-6132.

Two video training films, "Introduction to Life-Cycle Costing" and "Uncertainty and Risk, " are also available. These are two in a series of films on "Least-Cost Energy Decisions for Buildings." Additional video training films in the series are in preparation. The video films and companion workbooks can be ordered from Video Transfer, Inc., 5709-B Arundel Avenue, Rockville, MD 20852, Tel (301) 881-0270.

Further information on the Federal Energy Management Program can be obtained from the Federal Energy Management Program Staff, Office of the Assistant Secretary for Conservation and Renewable Energy, U.S. Department of Energy. Please direct communication to: FEMP, CE 10.1, U.S. Department of Energy, 1000 Independence Avenue, SW, Washington, DC 20585. 


\begin{abstract}
This is the 1994 annual edition of energy prices and discount factors for performing life-cycle cost analyses of energy conservation and renewable energy projects. It supports the Federal life-cycle costing methodology by updating the energy price projections and discount factors that are described, explained, and illustrated in NIST Handbook 135 (HB 135). It supports private-sector life-cycle cost analysis by updating the energy price indices that are described, explained, and illustrated in NBS Special Publication 709 (SP 709). It also supports the Energy Conservation Mandatory Performance Standards for New Federal Residential Buildings (10 CFR 435) by providing a table of factors for updating appliance label values.
\end{abstract}




\section{ACKNOWLEDGMENTS}

The author wishes to thank Mr. K. Dean DeVine of the Federal Energy Management Program of the U.S. Department of Energy (DOE) for his continued support and direction of this work. Appreciation is extended to Mr. Mark E. Rodekohr, Director, and Ms. Mary Joyce, Economist, of the Energy Demand and Integration Division of the DOE Energy Information Administration, for providing the energy price projections upon which this report is based, and to Miss Jennifer E. Day for her assistance in preparing this report for publication. 


\section{CONTENTS}

Page

FOREWORD $\ldots \ldots \ldots \ldots \ldots \ldots \ldots \ldots \ldots \ldots \ldots \ldots \ldots \ldots \ldots \ldots$ iii

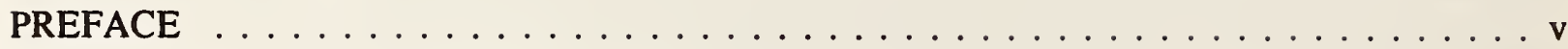

ABSTRACT $\ldots \ldots \ldots \ldots \ldots \ldots \ldots \ldots \ldots \ldots \ldots \ldots \ldots \ldots \ldots \ldots \ldots \ldots \ldots$

ACKNOWLEDGMENTS $\ldots \ldots \ldots \ldots \ldots \ldots \ldots \ldots \ldots \ldots$ ix

LIST OF TABLES $\ldots \ldots \ldots \ldots \ldots \ldots \ldots \ldots \ldots \ldots \ldots \ldots \ldots \ldots$

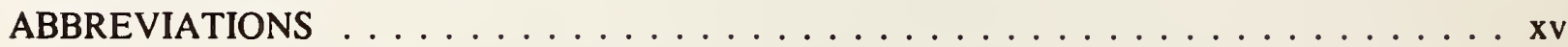

PART I: TABLES FOR FEDERAL LIFE-CYCLE COST ANALYSIS $\ldots \ldots \ldots \ldots \ldots \ldots \ldots$

A. Single Present Value and Uniform Present Value Discount Factors . . . . . . . . 1

B. Modified Uniform Present Value Discount Factors for Federal Use $\ldots \ldots \ldots \ldots 5$

C. Projected Average Fuel Price Indices and Escalation Rates for Federal Use . . . . 18

D. Factors for Updating Appliance Label Values . . . . . . . . . . . . . 35

PART II: TABLES FOR PRIVATE SECTOR LIFE-CYCLE COST ANALYSIS . . . . . 36

Projected Average Fuel Price Indices for Private Sector Use . . . . . . . . . 36 


\section{LIST OF TABLES}

Page

A-1. SPV factors for finding the present value of future single amounts (nonfuel) . . . 3

A-2. UPV factors for finding the present value of future annually recurring amounts (nonfuel)

Ba-1. UPV discount factors adjusted for fuel price escalation, by end-use sector and fuel type. Discount Rate $=3.1 \%$ (FEMP, FY 1994). Census Region 1 (Connecticut, Maine, Massachusetts, New Hampshire, New Jersey, New York, Pennsylvania, Rhode Island, Vermont $\ldots \ldots \ldots \ldots \ldots \ldots \ldots$

Ba-2. UPV discount factors adjusted for fuel price escalation, by end-use sector and fuel type. Discount Rate $=3.1 \%$ (FEMP, FY 1994). Census Region 2 (Illinois, Indiana, Iowa, Kansas, Michigan, Minnesota, Missouri, Nebraska, North Dakota, Ohio, South Dakota, Wisconsin) . . . . . . . . . . . . . . . . . .

Ba-3. UPV* discount factors adjusted for fuel price escalation, by end-use sector and fuel type. Discount Rate $=3.1 \%$ (FEMP, FY 1994). Census Region 3 (Alabama, Arkansas, Delaware, District of Columbia, Florida, Georgia, Kentucky, Louisiana, Maryland, Mississippi, North Carolina, Oklahoma, South Carolina, Tennessee, Texas, Virginia, West Virginia $\ldots \ldots \ldots \ldots \ldots \ldots$

Ba-4. UPV discount factors adjusted for fuel price escalation, by end-use sector and fuel type. Discount Rate $=3.1 \%$ (FEMP, FY 1994). Census Region 4 (Alaska, Arizona, California, Colorado, Hawaii, Idaho, Montana, Nevada, New Mexico, Oregon, Utah, Washington, Wyoming)

Ba-5. UPV discount factors adjusted for fuel price escalation, by end-use sector and fuel type. Discount Rate $=3.1 \%$ (FEMP, FY 1994). United States average . . . . 12

$\mathrm{Bb}-1$. UPV discount factors adjusted for fuel price escalation, by end-use sector and fuel type. Discount Rate $=4.0 \%$ (years $1-10$ ) and 4.5\% (years 11-30), (OMB Circular A-94). Census Region 1 (Connecticut, Maine, Massachusetts, New Hampshire, New Jersey, New York, Pennsylvania, Rhode Island, Vermont) . . . . . . . . . . . . . 13

$\mathrm{Bb}-2$. $\quad \mathrm{UPV}^{*}$ discount factors adjusted for fuel price escalation, by end-use sector and fuel type. Discount Rate $=4.0 \%$ (years $1-10$ ) and $4.5 \%$ (years 11-30), (OMB Circular A-94). Census Region 2 (Illinois, Indiana, Iowa, Kansas, Michigan, Minnesota, Missouri, Nebraska, North Dakota, Ohio, South Dakota, Wisconsin) . . . . . . . 14 


\section{LIST OF TABLES (continued)}

Page

Bb-3. UPV* discount factors adjusted for fuel price escalation, by end-use sector and fuel type. Discount Rate $=4.0 \%$ (years $1-10$ ) and 4.5\% (years 11-30), (OMB Circular A-94). Census Region 3 (Alabama, Arkansas, Delaware, District of Columbia, Florida, Georgia, Kentucky, Louisiana, Maryland, Mississippi, North Carolina, Oklahoma, South Carolina, Tennessee, Texas, Virginia, West Virginia) . . . . . 15

$\mathrm{Bb}-4$. UPV ${ }^{*}$ discount factors adjusted for fuel price escalation, by end-use sector and fuel type. Discount Rate $=4.0 \%$ (years $1-10$ ) and $4.5 \%$ (years 11-30), (OMB Circular A-94). Census Region 4 (Alaska, Arizona, California, Colorado, Hawaii, Idaho, Montana, Nevada, New Mexico, Oregon, Utah, Washington, Wyoming) . . . . . . 16

$\mathrm{Bb}$-5. $\quad \mathrm{UPV}^{*}$ discount factors adjusted for fuel price escalation, by end-use sector and fuel type. Discount Rate $=4.0 \%$ (years 1-10) and 4.5\% (years 11-30), (OMB Circular A-94). United States average ..................... . 17

Ca-1. Projected fuel price indices (excluding general inflation) by end-use sector and fuel type. Census Region 1 (Connecticut, Maine, Massachusetts, New Hampshire, New Jersey, New York, Pennsylvania, Rhode Island, Vermont) . . . . . . . . . . . . 20

Ca-2. Projected fuel price indices (excluding general inflation) by end-use sector and fuel type. Census Region 2 (Illinois, Indiana, Iowa, Kansas, Michigan, Minnesota, Missouri, Nebraska, North Dakota, Ohio, South Dakota, Wisconsin) . . . . . . 22

Ca-3. Projected fuel price indices (excluding general inflation) by end-use sector and fuel type. Census Region 3 (Alabama, Arkansas, Delaware, District of Columbia, Florida, Georgia, Kentucky, Louisiana, Maryland, Mississippi, North Carolina, Oklahoma, South Carolina, Tennessee, Texas, Virginia, West Virginia) . . . . . 24

Ca-4. $\quad$ Projected fuel price indices (excluding general inflation) by end-use sector and fuel type. Census Region 4 (Alaska, Arizona, California, Colorado, Hawaii, Idaho, Montana, Nevada, New Mexico, Oregon, Utah, Washington, Wyoming) . . . . . 26

Ca-5. Projected fuel price indices (excluding general inflation) by end-use sector and fuel type. United States average ... . . . . . . . . . . . . . . 28

$\mathrm{Cb}-1$. Projected average fuel price escalation rates exclusive of general price inflation, by end-use sector and fuel type (percentage change compounded annually). Census Region 1 (Connecticut, Maine, Massachusetts, New Hampshire, New Jersey, New York, Pennsylvania, Rhode Island, Vermont $\ldots \ldots \ldots \ldots$. . . . . . . 30 


\section{LIST OF TABLES (continued)}

Page

Cb-2. Projected average fuel price escalation rates exclusive of general price inflation, by end-use sector and fuel type (percentage change compounded annually). Census Region 2 (Illinois, Indiana, Iowa, Kansas, Michigan, Minnesota, Missouri, Nebraska, North Dakota, Ohio, South Dakota, Wisconsin) . . . . . . . . . . . . 31

Cb-3. Projected average fuel price escalation rates exclusive of general price inflation, by end-use sector and fuel type (percentage change compounded annually). Census Region 3 (Alabama, Arkansas, Delaware, District of Columbia, Florida, Georgia, Kentucky, Louisiana, Maryland, Mississippi, North Carolina, Oklahoma, South Carolina, Tennessee, Texas, Virginia, West Virginia) . . . . . . . . . 32

Cb-4. Projected average fuel price escalation rates exclusive of general price inflation, by end-use sector and fuel type (percentage change compounded annually). Census Region 4 (Alaska, Arizona, California, Colorado, Hawaii, Idaho, Montana, Nevada, New Mexico, Oregon, Utah, Washington, Wyoming) . . . . . . . . . 33

Cb-5. Projected average fuel price escalation rates exclusive of general price inflation, by end-use sector and fuel type (percentage change compounded annually). United

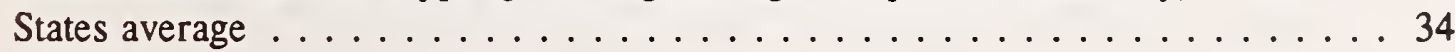

D. Factors for updating appliance label values . . . . . . . . . . . . . 35

S-1. $\quad$ Projected fuel price indices with assumed general price inflation rates of 3, 4, 5, and $6 \%$, by end-use sector and fuel type. Census Region 1 (Connecticut, Maine, Massachusetts, New Hampshire, New Jersey, New York, Pennsylvania, Rhode Island, Vermont) $\ldots \ldots \ldots . \ldots \ldots$

S-2. $\quad$ Projected fuel price indices with assumed general price inflation rates of $3,4,5$, and $6 \%$, by end-use sector and fuel type. Census Region 2 (Illinois, Indiana, Iowa, Kansas, Michigan, Minnesota, Nebraska, North Dakota, Ohio, South Dakota,

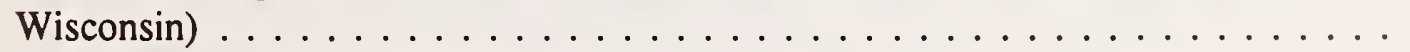

S-3. Projected fuel price indices with assumed general price inflation rates of 3, 4, 5, and $6 \%$, by end-use sector and fuel type. Census Region 3 (Alabama, Arkansas, Delaware, District of Columbia, Florida, Georgia, Kentucky, Louisiana, Maryland, Mississippi, North Carolina, Oklahoma, South Carolina, Tennessee, Texas, Virginia, West Virginia) 


\section{LIST OF TABLES (continued)}

S-4. $\quad$ Projected fuel price indices with assumed general price inflation rates of 3, 4, 5, and $6 \%$, by end-use sector and fuel type. Census Region 4 (Alaska, Arizona, California, Colorado, Hawaii, Idaho, Montana, Nevada, New Mexico, Oregon, Utah, Washington, Wyoming) . . . . . . . . . . . . . . . . 44

S-5. Projected fuel price indices with assumed general price inflation rates of 3, 4, 5, and $6 \%$, by end-use sector and fuel type. United States average . . . . . . . 46 


\section{ABBREVIATIONS}

$\begin{array}{lll}\text { COAL } & - & \text { Steam Coal } \\ \text { DIST } & - & \text { Distillate Oil } \\ \text { ELEC } & - & \text { Electricity } \\ \text { FY } & - & \text { Fiscal Year } \\ \text { GASLN } & - & \text { Gasoline } \\ \text { LCC } & - & \text { Life-Cycle Cost } \\ \text { LPG } & - & \text { Liquefied Petroleum Gas } \\ \text { N } & - & \text { Number of Discount Periods (in Years) } \\ \text { NTGAS } & - & \text { Natural Gas } \\ \text { RESID } & - & \text { Residual Oil } \\ \text { SPV } & - & \text { Single Present Value (Factor) } \\ \text { UPV } & - & \text { Uniform Present Value (Factor) } \\ \text { UPV* } & - & \text { Modified Uniform Present Value (Factor) }\end{array}$





\section{PART I: TABLES FOR FEDERAL LIFE-CYCLE COST ANALYSIS}

\section{A. Single Present Value and Uniform Present Value Discount Factors}

Table A-1 presents the single present value (SPV) factors for finding the present value of future nonfuel, nonannually recurring amounts, such as repair and replacement costs and salvage values. The formula for finding the present value $(\mathrm{P})$ of a future amount $(\mathrm{F})$ is the following:

$$
P=F \times \frac{1}{(1+d)^{N}}=F \times S P V_{N},
$$

where $\mathrm{d}=$ discount rate, and

$\mathrm{N}$ = number of periods, such as years, until $\mathrm{F}$ occurs.

Table A-2 presents uniform present value (UPV) factors for finding the present value of future nonfuel amounts recurring annually, such as routine maintenance costs. The formula for finding the present value $(\mathrm{P})$ of an annually recurring uniform amount $(\mathrm{A})$ is the following:

$$
P=A \times \frac{(1+d)^{N}-1}{d(1+d)^{N}}=A \times U P V_{N},
$$

where $\mathrm{d}=$ discount rate, and

$\mathrm{N}$ = number of periods, such as years, over which A recurs.

In Tables A-1 and A-2 the factors are given for both the FEMP and the OMB Circular A-94 discount rates. The factors based on the FEMP discount rate are for finding the present value of future amounts associated with Federal energy conservation and renewable energy projects. The factors based on the OMB discount rates are for finding the present value of future amounts associated with most other Federal projects (except those specifically exempted from OMB Circular A-94). Both the FEMP and the OMB discount rates used in these Tables are defined for Federal use to be "real" rates exclusive of general price inflation. The factors are applied as multipliers to future amounts which are stated in "constant" dollars, that is, exclusive of general price inflation.

\section{Examples of How to Use the Factors:}

SPV(FEMP): To compute the present value of a replacement cost expected to occur in the 8th year for an energy efficient heating system, go to Table A-1, find the 3.1\% SPV factor for year $8(0.78)$, and multiply the factor by today's replacement cost. "Today's" replacement cost is the cost as of the date of analysis. 
SPV(OMB, Short-term): To compute the present value of a repair cost in the 5th year for a floor covering (non-energy related), go to Table A-1, find the $4.0 \%$ SPV factor for year $5(0.82)$, and multiply the factor by today's repair cost.

SPV(OMB, Long-term): To compute the present value of a repair cost in the 15th year for a floor covering (non-energy related), go to Table A-1, find the 4.5\% SPV factor for year $15(0.52)$, and multiply the factor by today's repair cost.

UPV(FEMP): To compute the present value of an annually recurring maintenance cost for a renewable energy system over 20 years, go to Table A-2, find the $3.1 \%$ UPV factor for 20 years (14.74), and multiply the factor by the annual maintenance cost stated in today's dollars.

UPV(OMB, Short-term): To compute the present value of annually recurring costs of office cleaning over 10 years (for a project not primarily related to energy conservation), go to Table A-2, find the $4.0 \%$ UPV factor for 10 years (8.11), and multiply the factor by the annual cleaning cost stated in today's dollars.

UPV(OMB, Long-term): To compute the present value of annually recurring costs of office cleaning over 25 years (for a project not primarily related to energy conservation), got to Table A-2, find the $4.5 \%$ UPV factor for 25 years (14.83), and multiply the factor by the annual cleaning cost stated in today's dollars.

NOTE: The UPV factors are generally applied to costs which recur annually in substantially the same amount (in constant dollars), e.g., routine operating and maintenance costs. These costs usually occur over the service period of the building life. If there is a planning/design/construction period before the service life begins, during which these annually costs do not occur, the appropriate UPV factor for the service period is the difference between the UPV factor for the entire study period and the UPV factor for the planning/design/construction period. For example, if the planning/design/construction period is 3 years and the service period is 25 years, for a total study period of 28 years, the appropriate UPV factor (from Table A-2, FEMP $3.1 \%$ discount rate) is $18.54-2.82=15.72$.

For further explanation and illustration of how to use these factors, see NIST Handbook 135. 
Table A-1. SPV factors for finding the present value of future single amounts (non-fuel).

Single Present Value (SPV) Factors

\begin{tabular}{c} 
Year of \\
occurrence \\
$(t)$ \\
\hline 1 \\
2 \\
3 \\
4 \\
5 \\
6 \\
7 \\
8 \\
9 \\
10 \\
11 \\
12 \\
13 \\
14 \\
15 \\
16 \\
17 \\
18 \\
19 \\
20 \\
21 \\
22 \\
23 \\
24 \\
25 \\
26 \\
27 \\
28 \\
29 \\
30 \\
-------
\end{tabular}

\begin{tabular}{l} 
FEMP FY 1994 \\
Discount rate \\
3.18 \\
\hline 0.97 \\
0.94 \\
0.91 \\
0.89 \\
0.86 \\
0.83 \\
0.81 \\
0.78 \\
0.76 \\
0.74 \\
0.71 \\
0.69 \\
0.67 \\
0.65 \\
0.63 \\
0.61 \\
0.60 \\
0.58 \\
0.56 \\
0.54 \\
0.53 \\
0.51 \\
0.50 \\
0.48 \\
0.47 \\
0.45 \\
0.44 \\
0.43 \\
0.41 \\
0.40 \\
$--0--1$ \\
--1
\end{tabular}

OMB Discount Rates /a

Short term/b Long Term/c

4.08 Long

---------

0.96

0.92

0.89

0.85

0.82

0.79

0.76

0.73

0.70

4.58

0.96

0.92

0.88

0.84

0.80

0.77

0.73

0.68

0.70

0.67

0.64

0.62

0.59

0.56

0.54

0.52

0.49

0.47

0.45

0.43

0.41

0.40

0.38

0.36

0.35

0.33

0.32

0.30

0.29

0.28

0.27

/a OMB discount rates as of March 1993. OMB rates are expected to be revised in February 1994.

/b Short-term discount rate based on OMB discount rate for 7-year study period.

/C Long-term discount rate based on OMB discount rate for 30-year study period. 
Table A-2. UPV factors for finding the present value of annually recurring uniform amounts (non-fuel).

\begin{tabular}{|c|c|c|c|}
\hline & & & \\
\hline $\begin{array}{l}\text { Year of } \\
\text { Occurrence } \\
(t)\end{array}$ & $\begin{array}{l}\text { FEMP FY } 1994 \\
\text { Discount rate } \\
3.18\end{array}$ & $\begin{array}{c}\text { OMB Discol } \\
\text { short term/b } \\
4.08\end{array}$ & $\begin{array}{c}\text { nt Rates/a } \\
\text { Long Term/c } \\
4.58\end{array}$ \\
\hline 1 & 0.97 & 0.96 & 0.96 \\
\hline 2 & 1.91 & 1.89 & 1.87 \\
\hline 3 & 2.82 & 2.78 & 2.75 \\
\hline 4 & 3.71 & 3.63 & 3.59 \\
\hline 5 & 4.57 & 4.45 & 4.39 \\
\hline 6 & 5.40 & 5.24 & 5.16 \\
\hline 7 & 6.21 & 6.00 & 5.89 \\
\hline 8 & 6.99 & 6.73 & 6.60 \\
\hline 9 & 7.75 & 7.44 & 7.27 \\
\hline 10 & 8.49 & 8.11 & 7.91 \\
\hline 11 & 9.20 & & 8.53 \\
\hline 12 & 9.89 & & 9.12 \\
\hline 13 & 10.57 & & 9.68 \\
\hline 14 & 11.22 & & 10.22 \\
\hline 15 & 11.85 & & 10.74 \\
\hline 16 & 12.47 & & 11.23 \\
\hline 17 & 13.06 & & 11.71 \\
\hline 18 & 13.64 & & 12.16 \\
\hline 19 & 14.20 & & 12.59 \\
\hline 20 & 14.74 & & 13.01 \\
\hline 21 & 15.27 & & 13.40 \\
\hline 22 & 15.78 & & 13.78 \\
\hline 23 & 16.27 & & 14.15 \\
\hline 24 & 16.75 & & 14.50 \\
\hline 25 & 17.22 & & 14.83 \\
\hline 26 & 17.67 & & 15.15 \\
\hline 27 & 18.11 & & 15.45 \\
\hline 28 & 18.54 & & 15.74 \\
\hline 29 & 18.95 & & 16.02 \\
\hline 30 & 19.35 & & 16.29 \\
\hline
\end{tabular}

/a OMB discount rates as of March 1993. OMB discount rates are expected to be revised in February 1994.

/b short-term discount rate based on OMB discount rate for 7-year study period.

/c Long-term discount rate based on OMB discount rate for 30 -year study period. 


\section{B. Modified Uniform Present Value Discount Factors for Federal Use (Based on the FEMP and OMB discount rates and DOE-projected rates of change in energy prices, both of which exclude general price inflation)}

This section presents "modified" uniform present value (UPV*) discount factors for the 4 Census regions and for the United States. The factors are modified in the sense that they incorporate the annual rate of energy price changes currently projected by DOE for the years 1994-2023. There are two sets of tables: the "Ba" tables present UPV factors based on the FEMP discount rate $(3.1 \%$ real), and the "Bb" tables present UPV" factors based on two OMB discount rates (4.0\% real for short-term study periods of 1 to 10 years, $4.5 \%$ real for long-term study periods of 11 to 30 years).

The factors presented in the "Ba" tables, based on the FEMP discount rate, are for calculating the present value of energy costs or savings accruing over 1 to 25 years and are to be used in life-cycle cost analyses of Federal energy conservation and renewable energy projects. Factors are reported in the "Ba" tables for 30 years to accommodate a planning/design/construction period of up to 5 years. (See "Examples of How to Use UPV* Factors" below for instructions on use with planning/design/construction periods.)

The factors presented in the "Bb" tables, based on the OMB discount rates, are for calculating the present value of energy costs or savings accruing over 1 to 30 years and are to be used for life-cycle cost analysis of the energy component of Federal projects that are not primarily for conserving energy or providing renewable energy.

Both sets of factors apply only to annual energy usage or savings that are the same each year over the service period that recur in uniform amounts. Refer to NIST Handbook 135 for evaluating annual energy amounts that vary in amount over time.

The UPV factors incorporate rates of change in energy prices computed from prices projected by the Energy Information Administration (EIA) of the U.S. Department of Energy. Projections at the national level to the year 2010 are reported in the Annual Energy Outlook 1993 (DOE/EIA0383(93)). Assumptions underlying the model used by EIA to project energy prices to the year 2010 are presented in Assumptions for the Annual Energy Outlook 1993 (DOE/EIA-0527(93)). Projections of world oil prices are used by EIA to project prices for related fuels for years 2011 to 2023 and are documented in Oil Market Simulation User's Manual (DOE/EIA-M028(91)).

The formula for finding the present value $(\mathrm{P})$ of future energy costs or savings is the following:

$$
P=A_{0} \times \sum_{t=1}^{N} \frac{I_{(1993+t)}}{(1+d)^{t}}=A_{o} \times U P V^{*}{ }_{N},
$$

where $A_{0}=$ base-year dollar cost of energy, i.e., the annual quantity of energy times its price in today's dollars;

$\mathrm{t}=$ counter used to designate each year, with $\mathrm{t}=1$ for the year 1994;

$\mathrm{N}=$ number of periods, e.g. years, over which energy costs or savings accrue; 


$\begin{array}{lll}\mathrm{I}_{(1993+1)} & = & \text { projected average fuel price index }{ }^{1} \text { given in Tables Ca-1 through Ca-5 } \\ \mathrm{d} & = & \text { for the year } 1993+\mathrm{t} \text { and }\end{array}$

\section{Examples of How to Use UPV* Factors:}

$\mathrm{UPV}^{*}$ (FEMP discount rate, no planning/design/construction period): To compute the present value of heating with distillate oil over 15 years for an energy-conserving design of a Federal office building in New Mexico, go to Table B-4a, find the UPV factor for commercial distillate for 15 years (13.60), and multiply the factor by the annual heating cost in today's dollars. The cost in "today's" dollars is the cost as of the date of analysis.

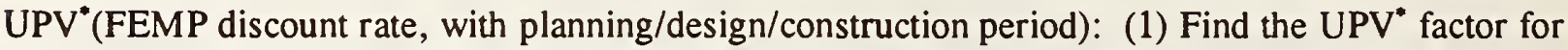
the combined lengths of the planning/design/construction period and the occupancy period (not to exceed 30 years), and (2) subtract from (1) the UPV ${ }^{*}$ factor for the planning/design/construction period alone. The difference is the UPV* factor for the years over which energy costs or savings accrue. For example, suppose an energy-conserving Federal office building in New York is being designed. It is expected to have a planning/design/construction period of 5 years, after which it will be occupied for at least 25 years. To compute the present value of natural gas costs over 25 years of occupancy, go to Table B-1a and find the UPV factors for commercial natural gas for 5 years (4.78) and for 30 years (24.69). The difference (19.91) is the UPV factor for natural gas costs over 25 years, beginning 5 years hence. Multiply 19.91 by the annual natural gas cost in today's dollars to calculate the present value of natural gas costs over the study period.

$\mathrm{UPV}^{*}(\mathrm{OMB}$ discount rates): To compute the present value of electricity costs over 30 years associated with the occupancy of a Federal office building in Ohio (where energy conservation is not a specific consideration in the LCC analysis), go to Table B-2b, find the UPV factor for commercial electricity for 30 years (15.64), and multiply by the annual electricity cost in today's dollars.

NOTE: Because the discount rate used to calculate the $\mathrm{Bb}$ tables (OMB discount rate) is different for years 1-10 (4.0\%) than for years 11-30 (4.5\%), these factors cannot be used with a planning/design/construction period as shown above for the Ba tables (FEMP discount rate). Use the BLCC or DISCOUNT computer program for this purpose. For further explanation of the use of UPV* factors, see NIST Handbook 135.

The data in the tables which follow are reported for the 4 Census regions and the U.S. average. Figure B-1 presents a map showing the states corresponding to the 4 Census regions. The Census regions do not include American Samoa, Canal Zone, Guam, Puerto Rico, Trust Territory of the Pacific Islands, or the Virgin Islands. Analysts of Federal projects in these areas should use data which are "reasonable under the circumstances," and may refer to the tables with U.S. average data for guidance.

${ }^{1}$ For greater precision, the UPV* factors reported in the $\mathrm{Ba}$ and $\mathrm{Bb}$ tables were computed using the unrounded form of the indices given in Tables Ca-1 through Ca-5. 


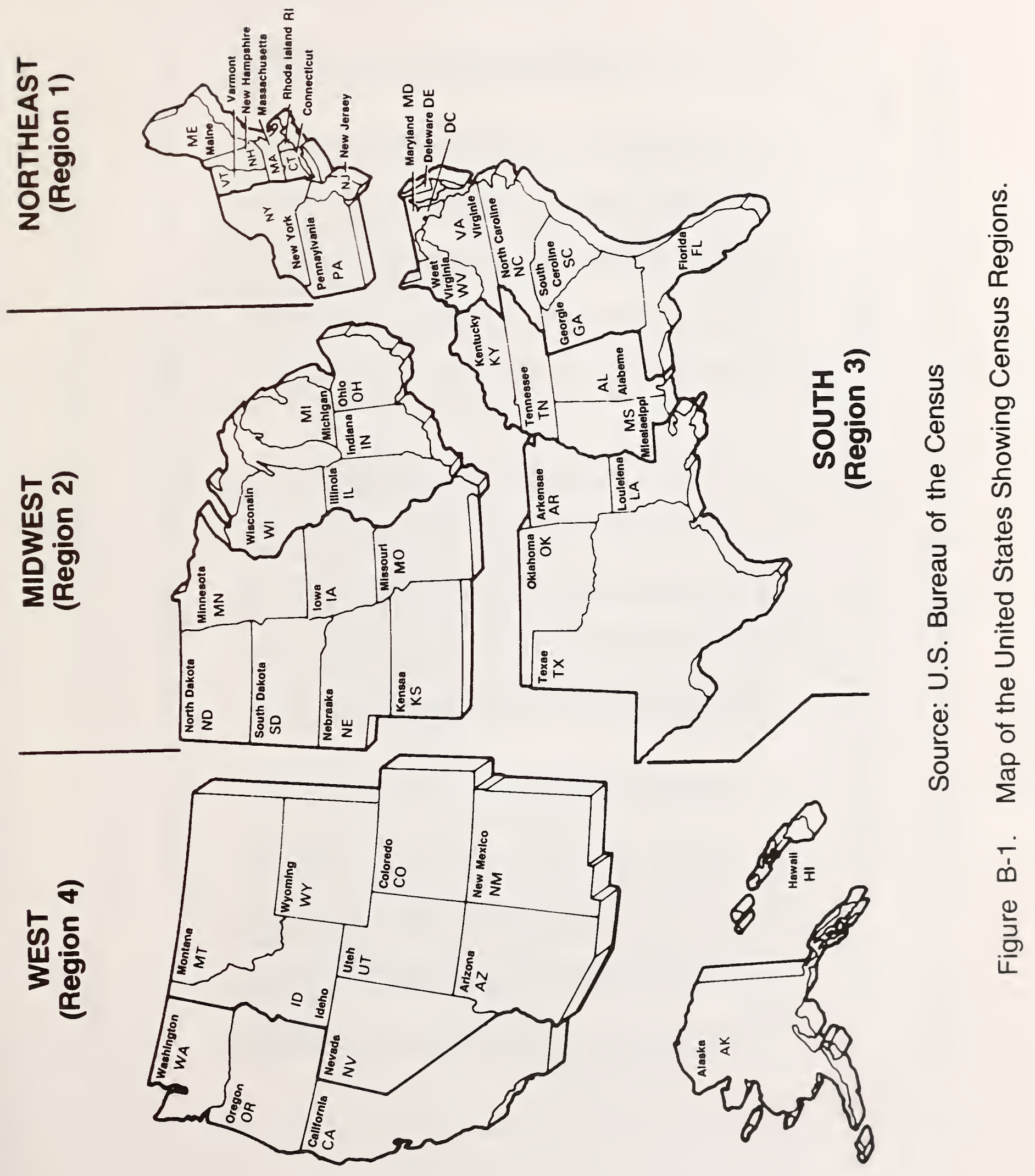




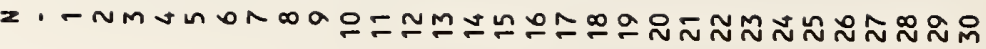

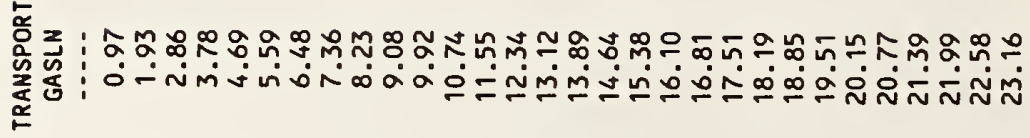

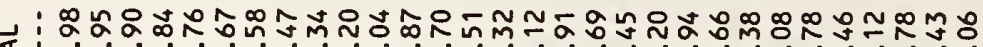

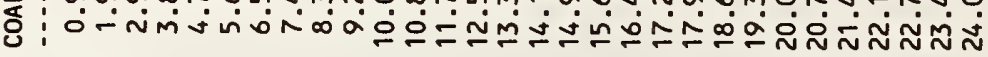

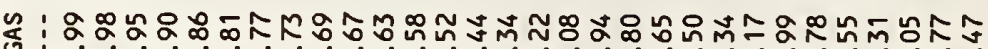
娄

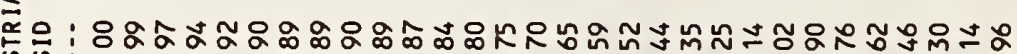
范 尊

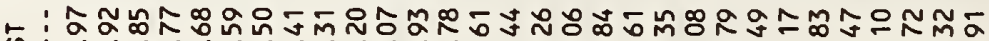
ล

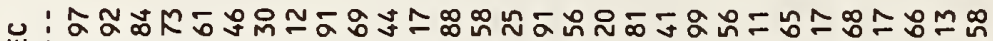

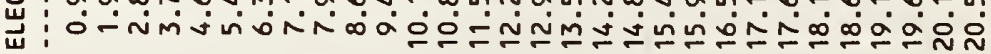

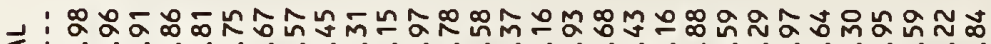
ठ

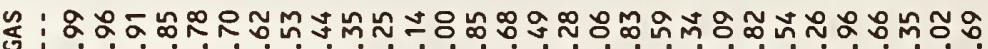
|

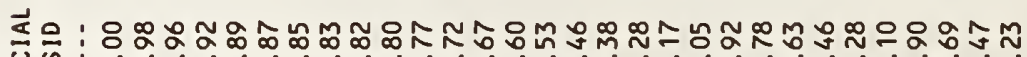

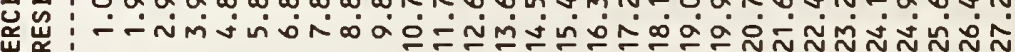
章

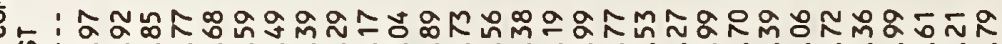
ڤ

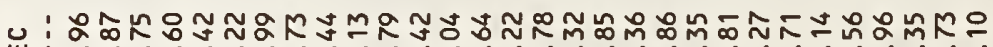
w

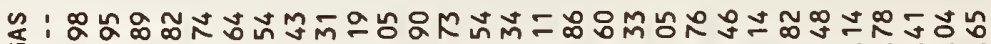
娄

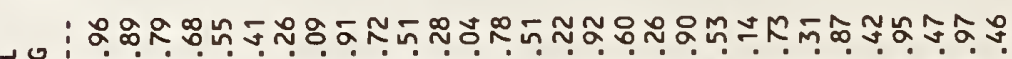

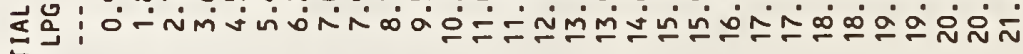

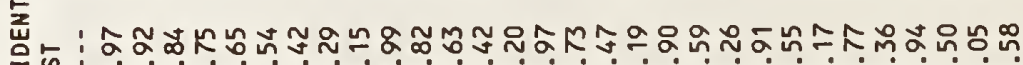

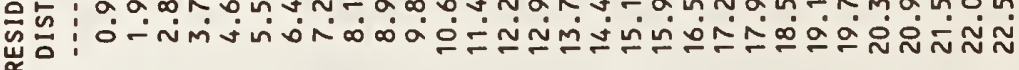

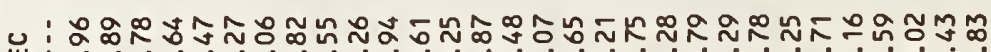
岂:

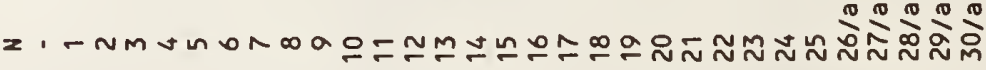




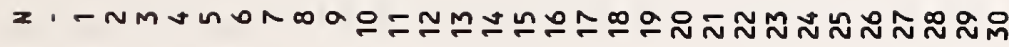

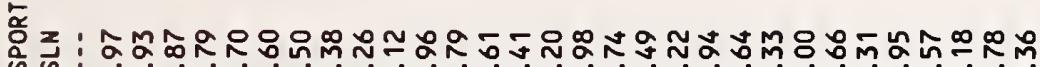

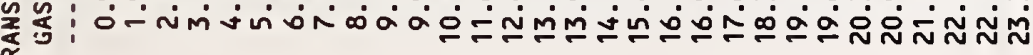

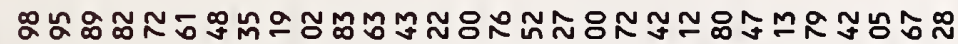

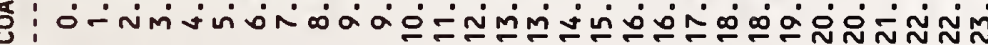

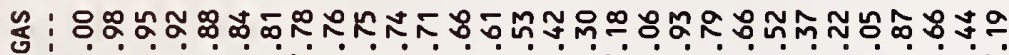

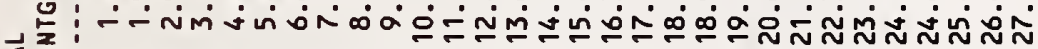
๙

㟧

—

ธ

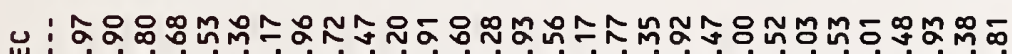

यّ山-

| ํำ

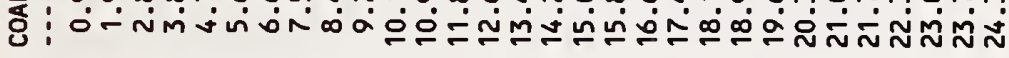

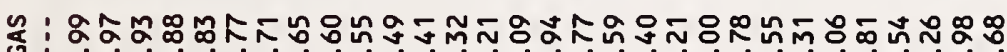

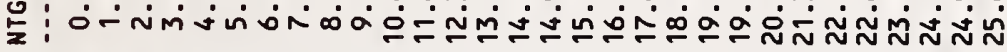

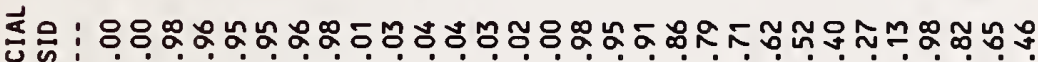

峞崖 -

突

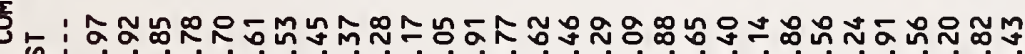

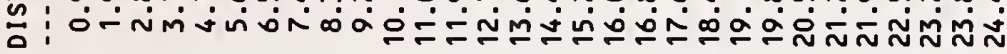

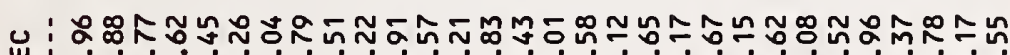

Ш

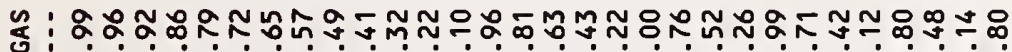

定

: ผ ผ

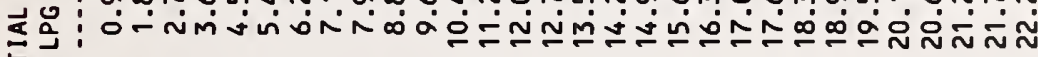

菤自

崫荡

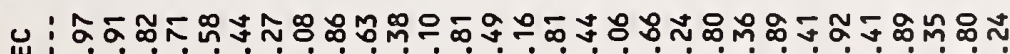

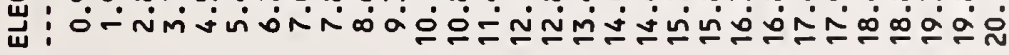

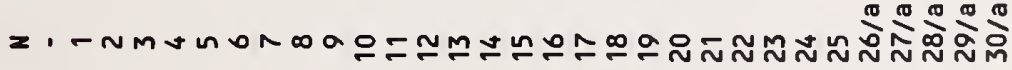




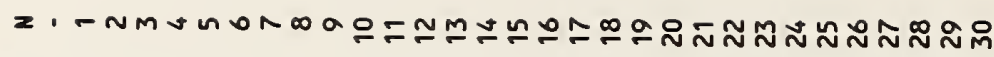

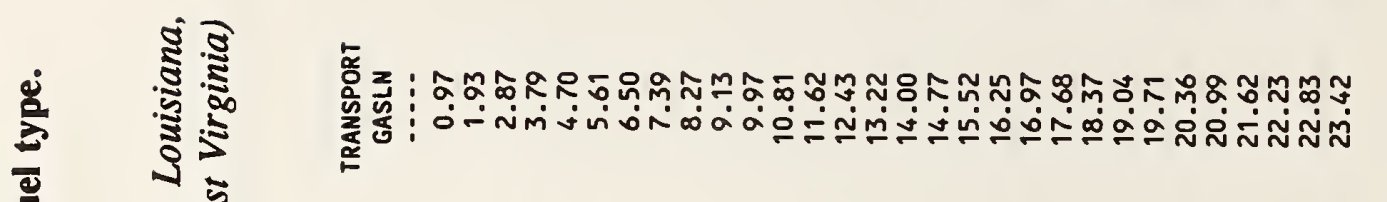

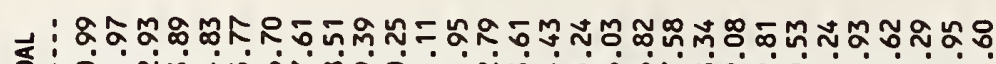

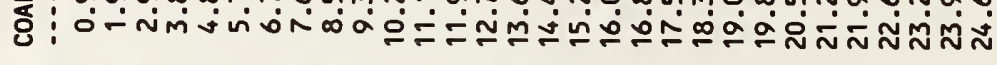

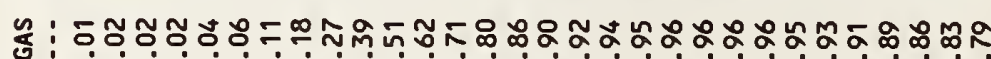
造: -

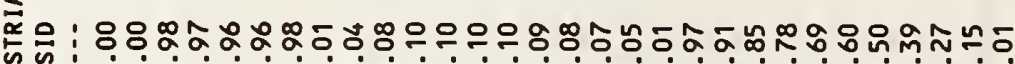

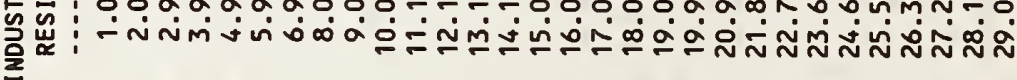
โ

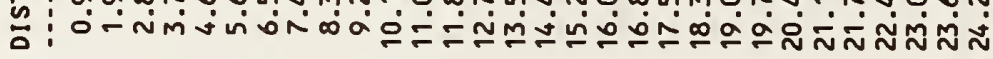

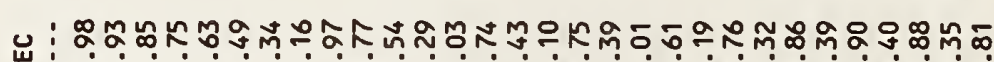

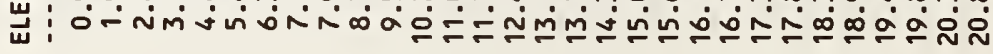

|

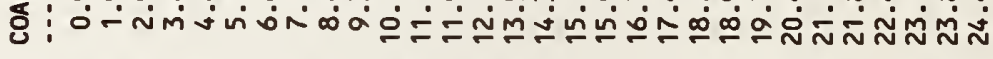

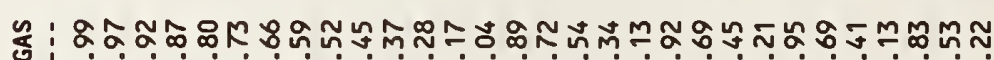
L

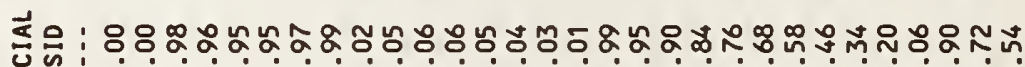
崖

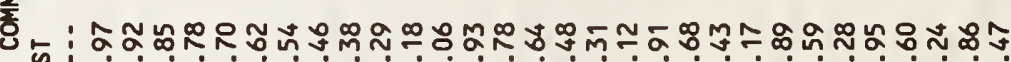
○)

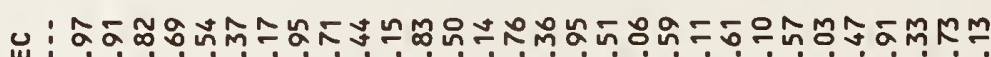

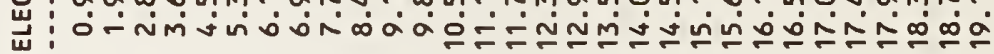

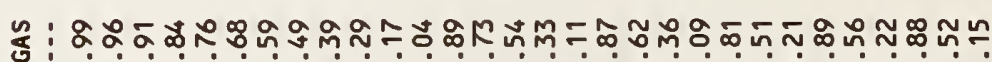

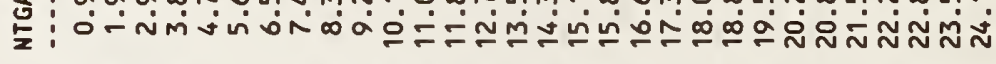

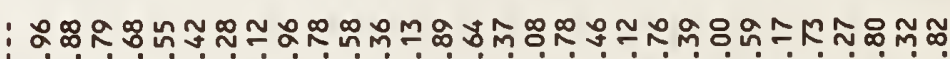

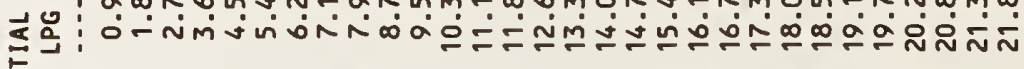
营 崖点 ㄴ

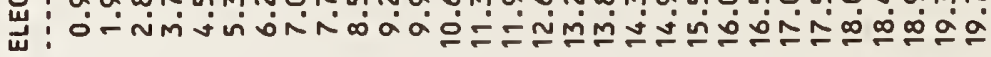

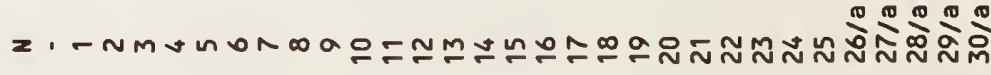




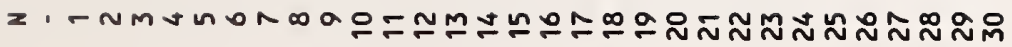

ํㅜㅇz: :

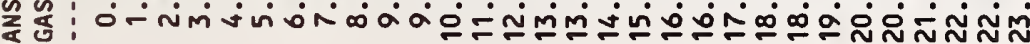

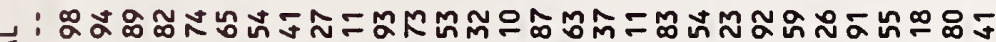

ปั-

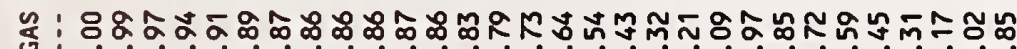

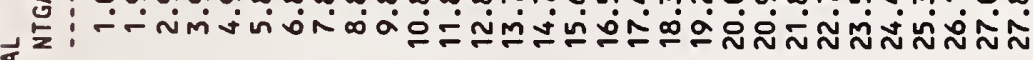

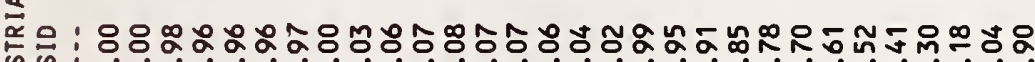
气岱

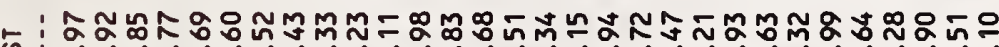

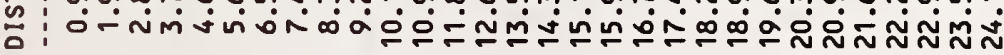

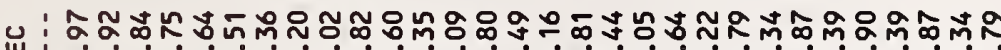

岁:

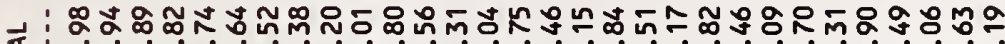

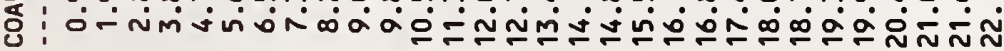

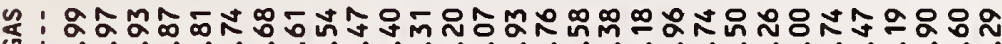

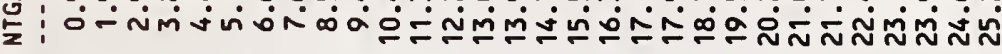

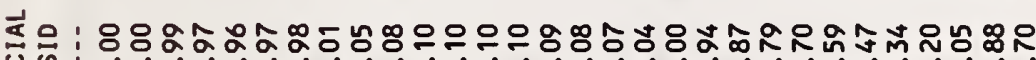
巡 害

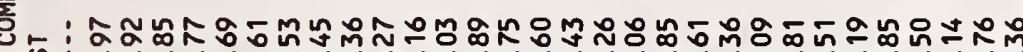

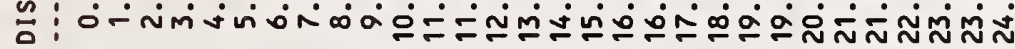

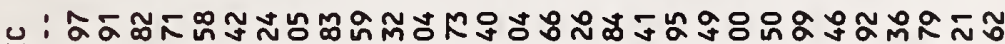
यั山

: : :

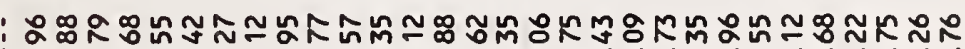

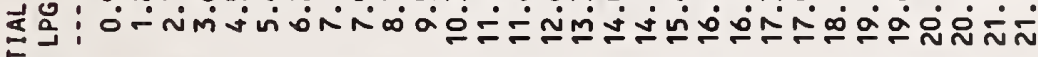

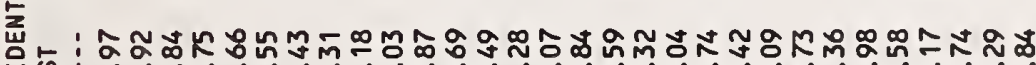
岩菏

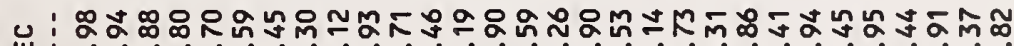
岀 O-

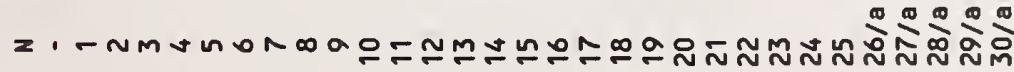


スー -

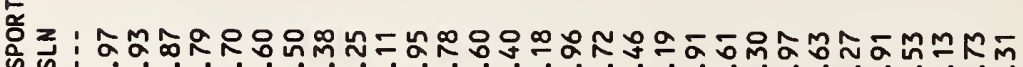

造:

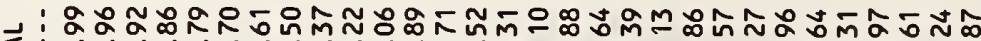
ठ์

น์

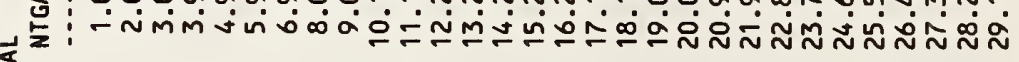

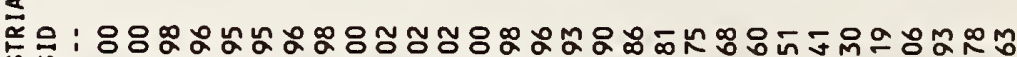
出: เ a

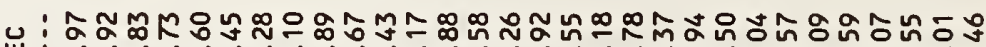
u

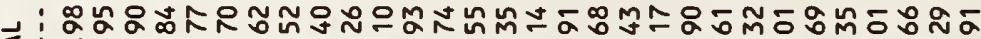

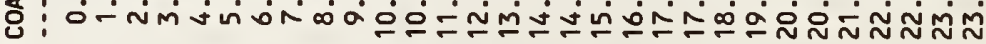

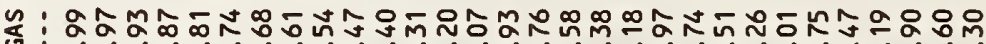

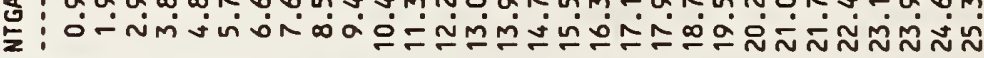

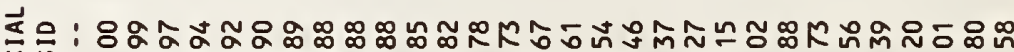

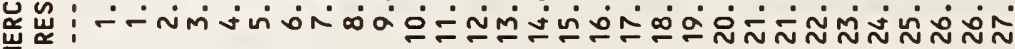
害

เ

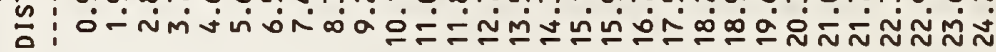

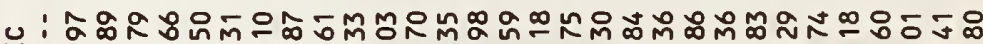
ü

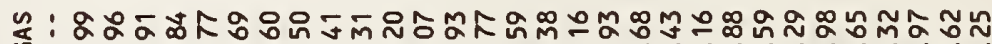
它

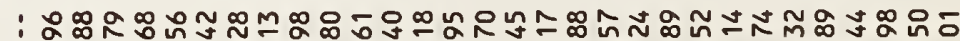

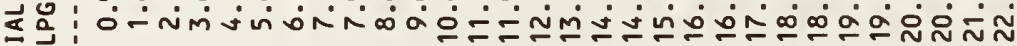

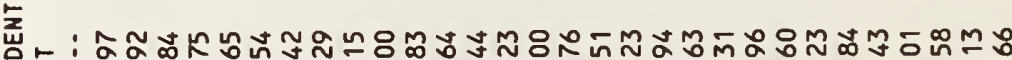
营泀

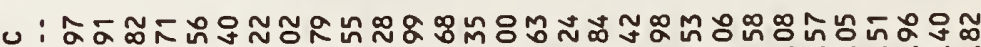

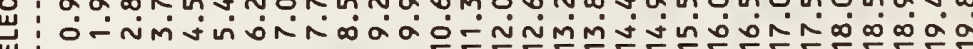

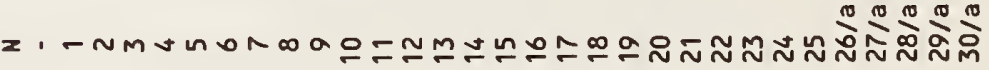


x. - N

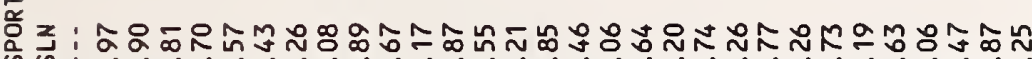

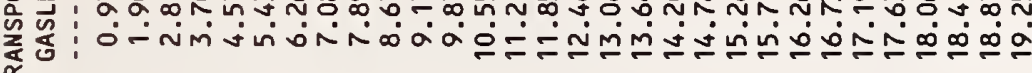

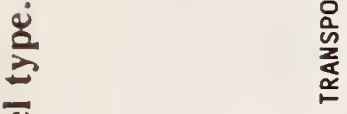

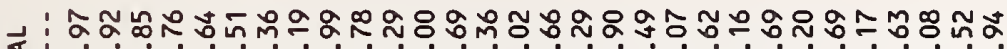
沗

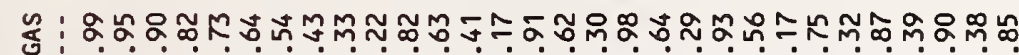
ـ :-

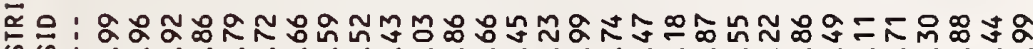

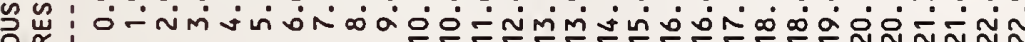

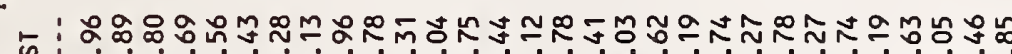
ด O-

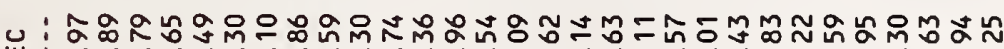
ü 0 -

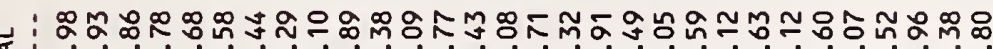
व̊-

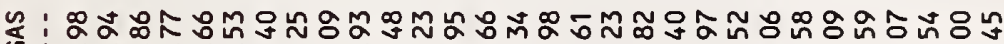
: O-

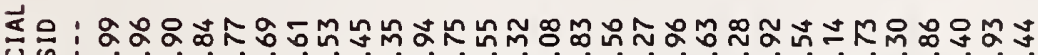
峞 0窟

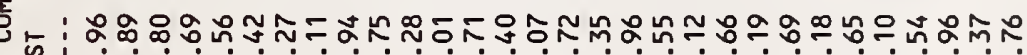
o

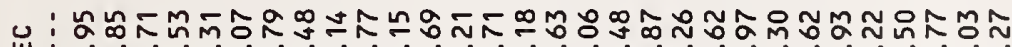
u

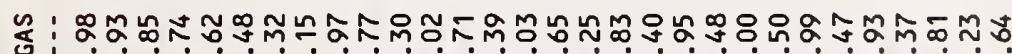
L

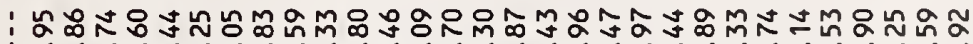
区은 胥

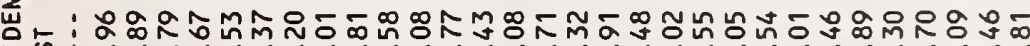

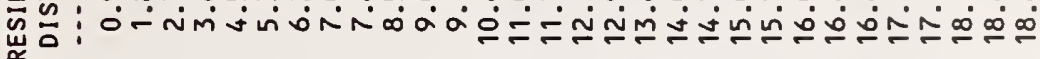
ب :

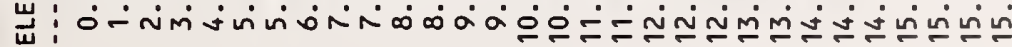

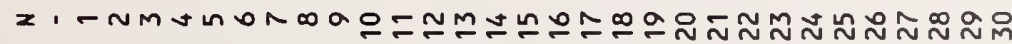




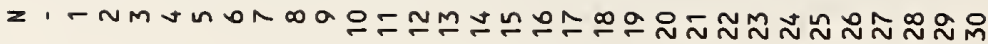

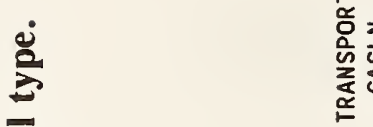

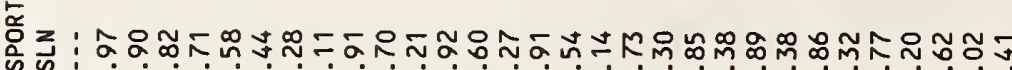

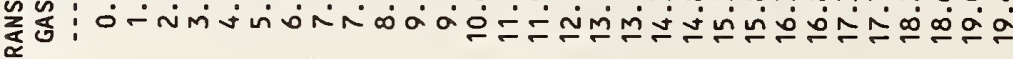

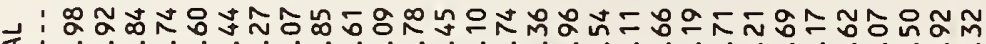

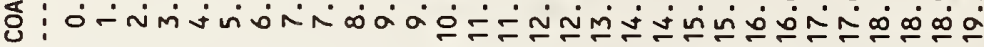

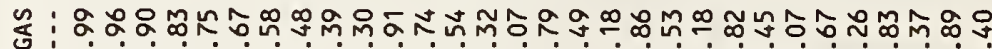

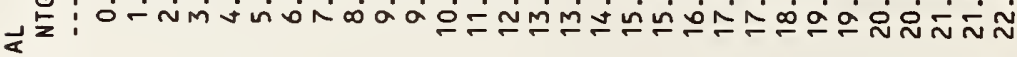

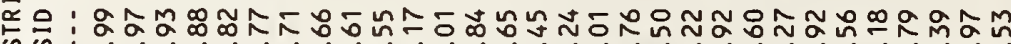

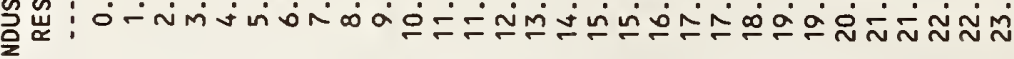

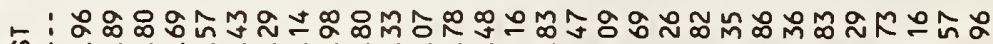

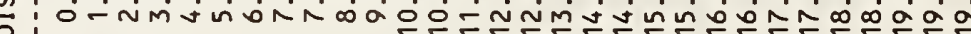

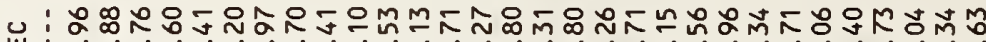
แ

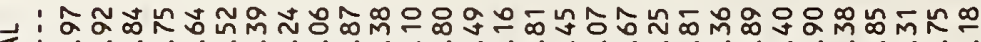
ठั 눈

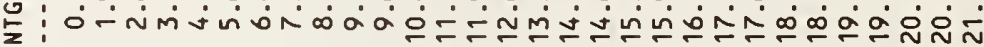
区은

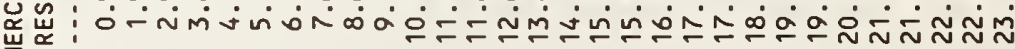
章

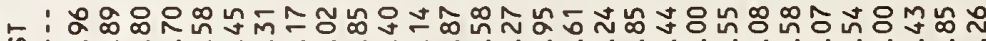

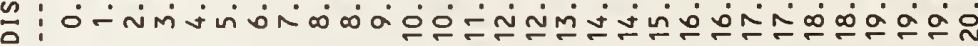

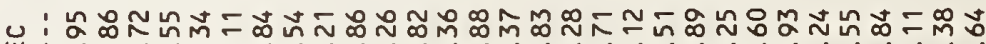

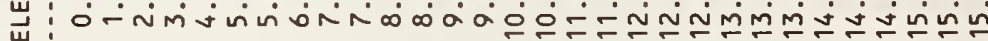

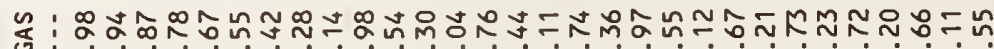

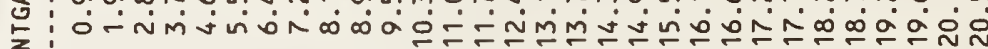

แ ผ ผ

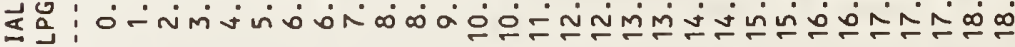
胥

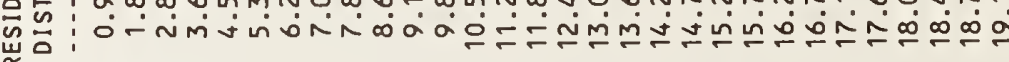

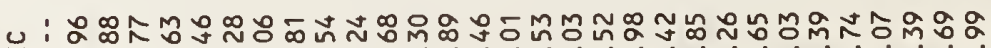
ǘnm

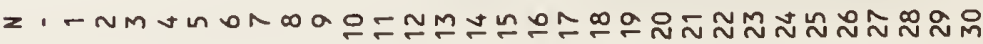




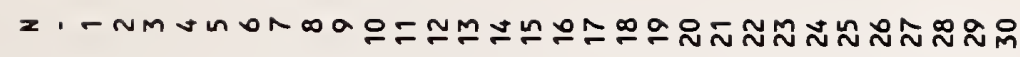

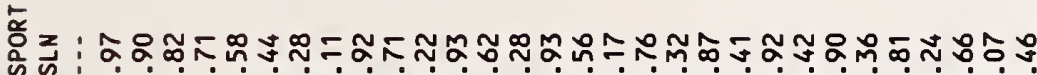
选:

ᄀ :

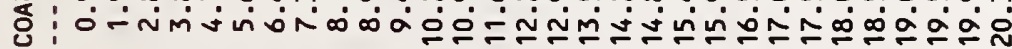

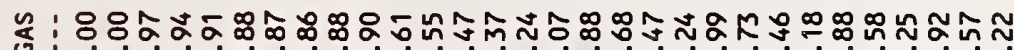
起: -

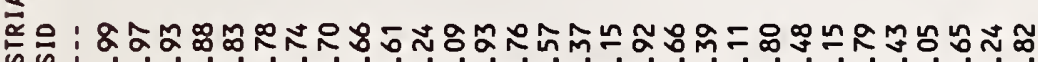

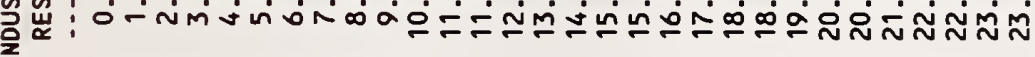

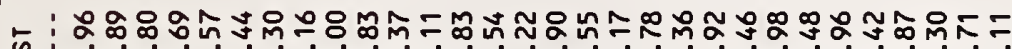

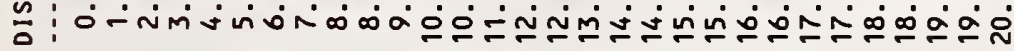

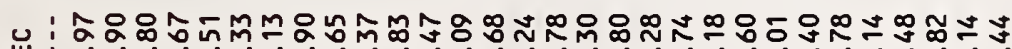
யّ

: :

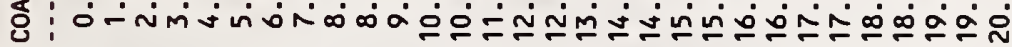

ํㅝㅁ: z: 0 -

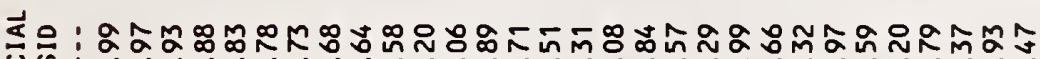

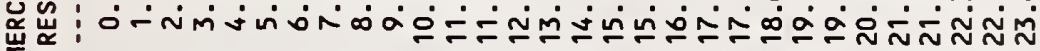

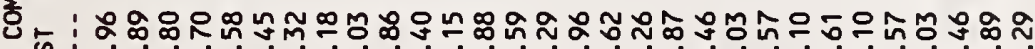

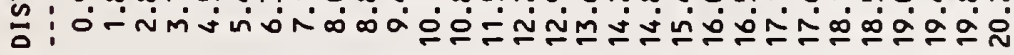

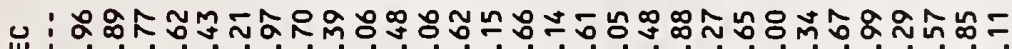
य

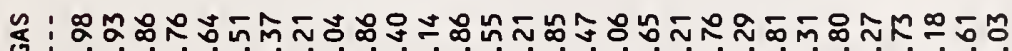
z:

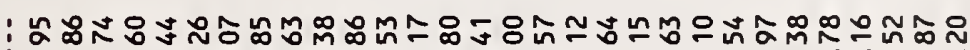

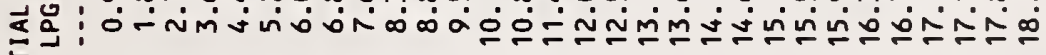

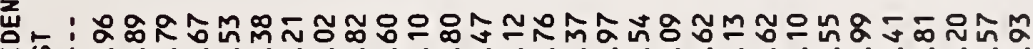

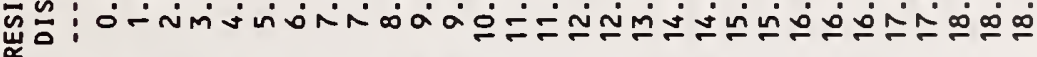

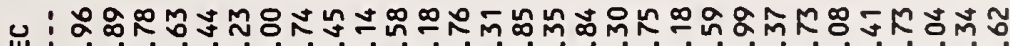
ய z. - M U แ 


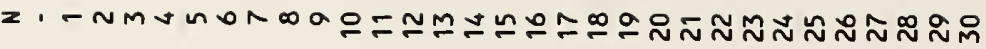

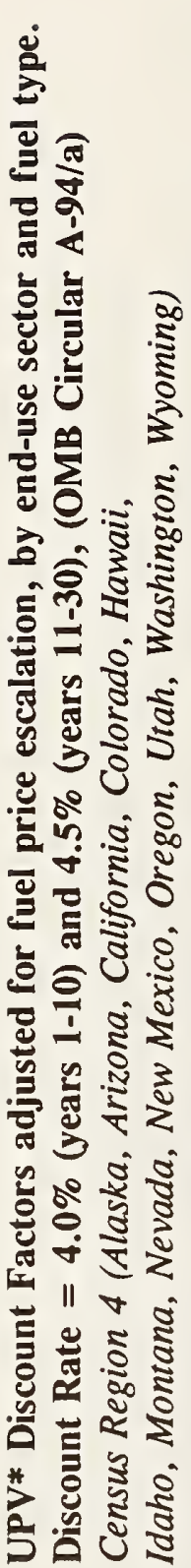

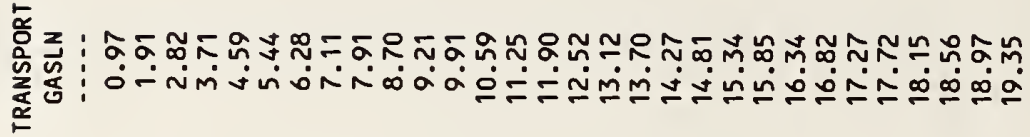

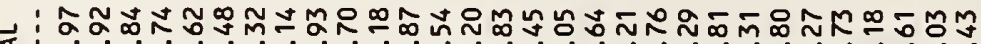

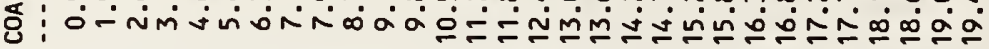

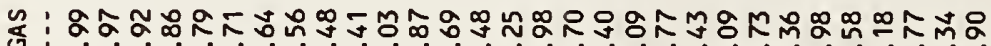

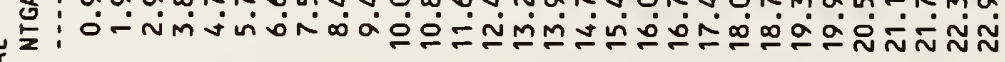

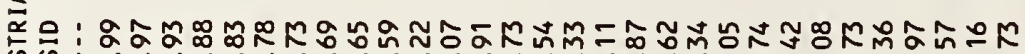

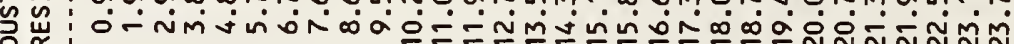
을

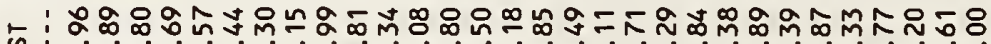
ธ О-

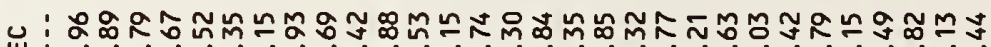
य

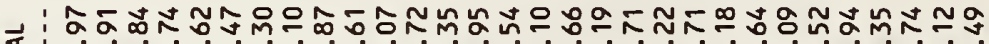

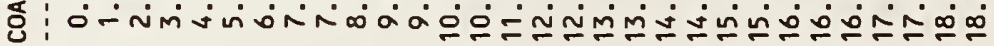

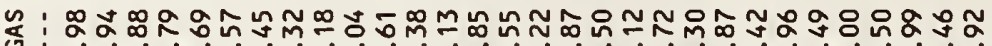
L

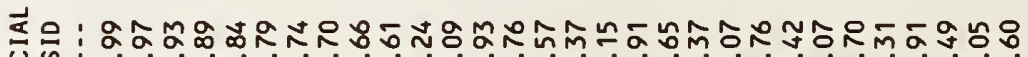

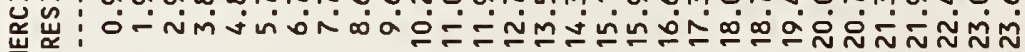
蛋

总。 ธด-

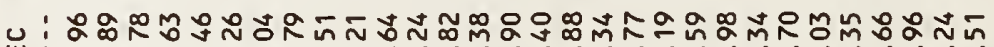

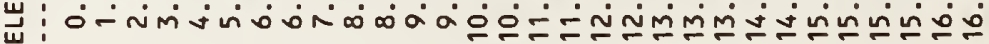

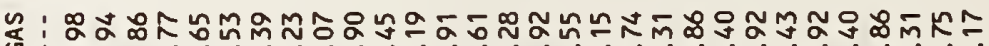

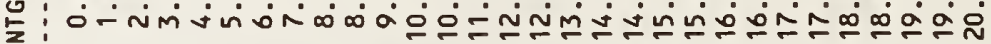

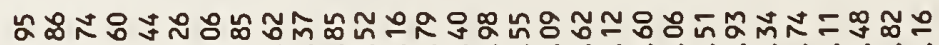

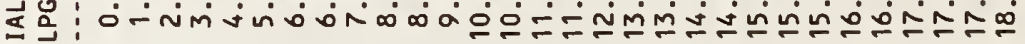

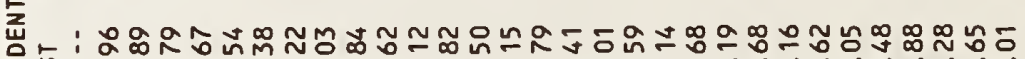

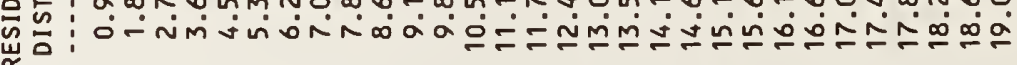

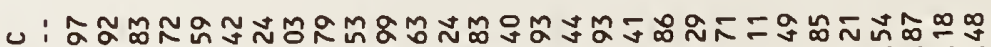

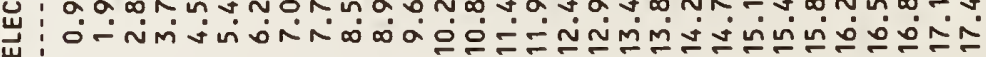

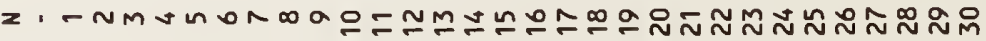




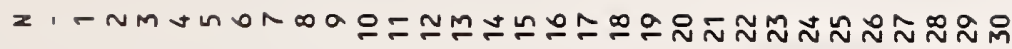

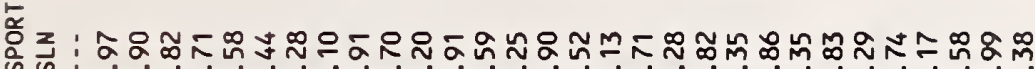

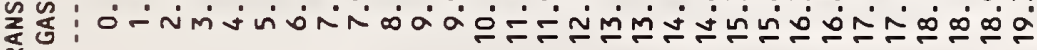

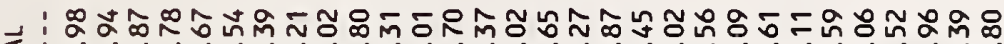
ठั

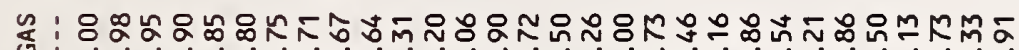

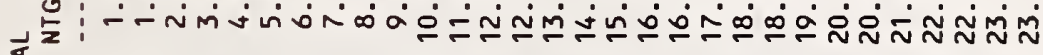

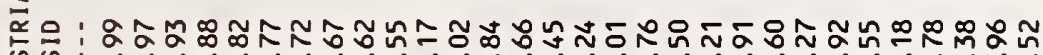

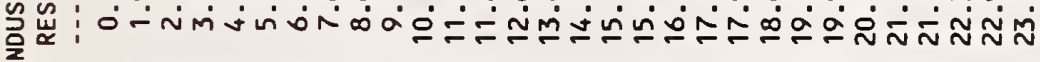
ᄂ

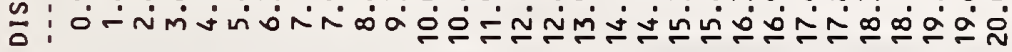

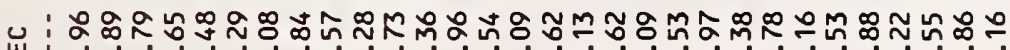
यั山

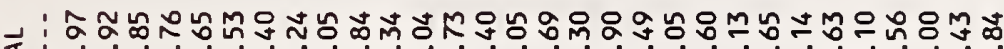

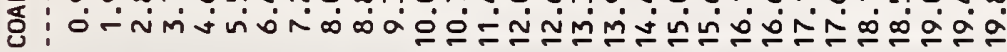
造: 定

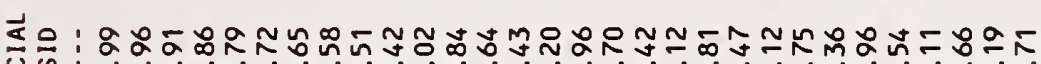

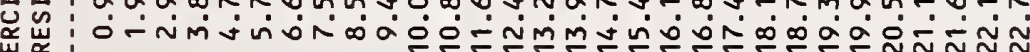
峷

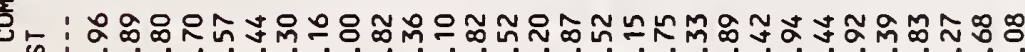
๑

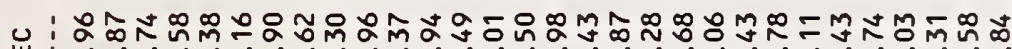
यั山

๒ : ロ L 0 -

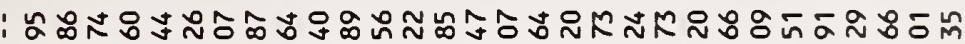

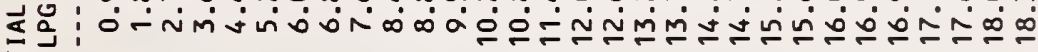

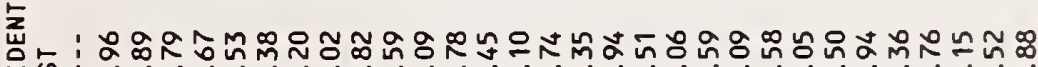

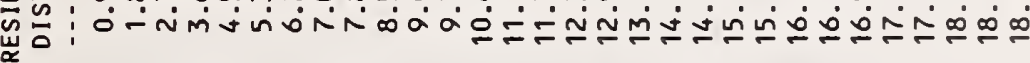

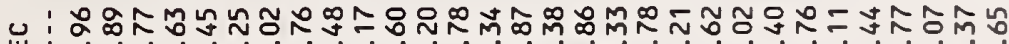
य

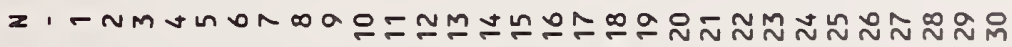




\section{Projected Average Fuel Price Indices and Escalation Rates for Federal Use (Indices and escalation rates exclude general price inflation)}

Table C, "Regional and U.S. average base-year fuel prices by end-use sector and major fuel," has been discontinued. Use your actual energy prices as of the date the analysis is performed as the starting point for estimating present value energy costs.

Tables Ca-1 through $\mathrm{Ca}-5$ present projected average fuel price indices for the 4 Census regions and for the United States. These are multipliers which when applied to today's prices provide estimates of the corresponding future-year prices in constant base-year dollars (beginning of year 1994). The indices reflect end-of-year prices. End-of-year indices are needed because energy prices are discounted from the end of the year in calculating the UPV ${ }^{*}$ factors. Constant dollar prices are needed when discounting is performed with a real discount rate (i.e., one which does not include general price inflation).

\section{Example of How to Use the Indices:}

To estimate the price of industrial steam coal at the end of year 2005 in Connecticut, go to Table Ca-1, find the year 2005 index for industrial steam coal (1.20), and multiply by the price for industrial steam coal in Connecticut at the beginning of 1994.

For further explanation of how to use these tables, see NIST Handbook 135.

Tables $\mathrm{Cb}-1$ through $\mathrm{Cb}-5$ present the projected average fuel price escalation rates (percentage change compounded annually) for seven selected periods from 1993 to 2023 for the 4 Census regions and for the United States. Note that these are "real" rates exclusive of general price inflation. Their use results in prices expressed in constant dollars.

The average fuel escalation rates consolidate the information provided by the indices in the $\mathrm{Ca}$ tables so that trends in projected price changes can be seen at a glance. They are provided primarily to accommodate those who use computer programs which require escalation rates as inputs.

Unless there is a compelling reason to use escalation rates, it is recommended that you use the indices in the Ca tables when you need estimates of future-year energy prices, since the indices include year-to-year information rather than averages over a number of years. 


\section{Example of How to Use the Escalation Rates:}

To estimate the price of residential distillate at the end of $1998\left(\mathrm{p}_{98}\right)$ in Wyoming using the escalation rates, go to Table Cb-4 and find the 1993-1995 and 1995-2000 escalation rates for residential distillate $(0.3 \%$ for two years and $1.7 \%$ for three years, respectively). Enter these values and the price of residential distillate in Wyoming at the end of $1993\left(\mathrm{p}_{93}\right)^{2}$ into the following formula. Then solve for the 1998 energy price (stated in end-of-year 1993 dollars):

$$
\begin{aligned}
p_{98} & =p_{93} \times\left(1+e_{1}\right)^{k_{1}} \times\left(1+e_{2}\right)^{k_{2}} \\
& =p_{93} \times(1+0.003)^{2} \times(1+0.017)^{3} \\
& =p_{93} \times 1.0582
\end{aligned}
$$

where $p_{y}=$ Price at end of year $y$;

$e_{i}=$ Annual compound escalation rate for period $\mathrm{i}$ from the $\mathrm{Cb}$ tables (in decimal form); and

$\mathrm{k}_{\mathrm{i}}=$ Number of years over which escalation rate $\mathrm{e}_{\mathrm{i}}$ occurs.

The data in the tables which follow are reported for the 4 Census regions and the U.S. average. Figure B-1 presents a map showing the states corresponding to the 4 Census regions. The Census regions do not include American Samoa, Canal Zone, Guam, Puerto Rico, Trust Territory of the Pacific Islands, or the Virgin Islands. Analysts of Federal projects in these areas should use data which are "reasonable under the circumstances," and may refer to the tables with U.S. average data for guidance.

${ }^{2}$ The price at the end of year 1993 is the same as the price at the beginning of 1994 . End-ofyear 1993 is used here to maintain consistent notation. 


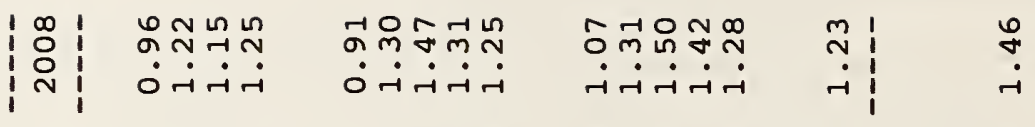

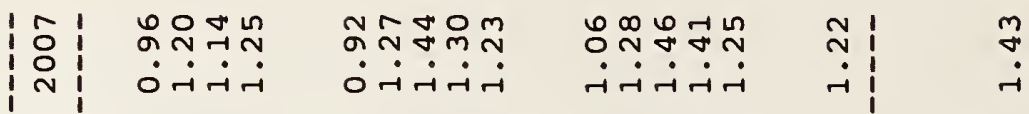

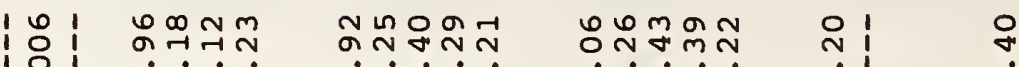

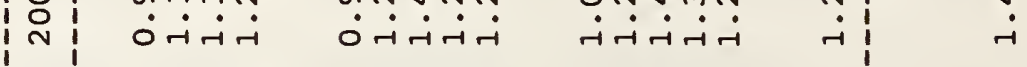

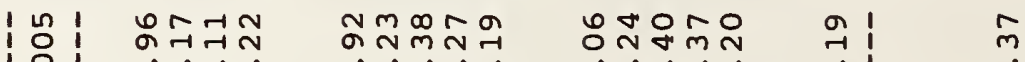

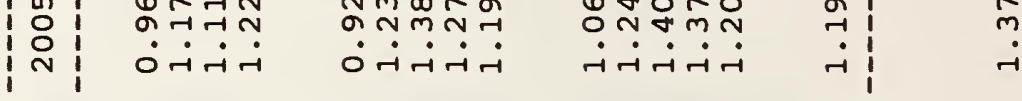

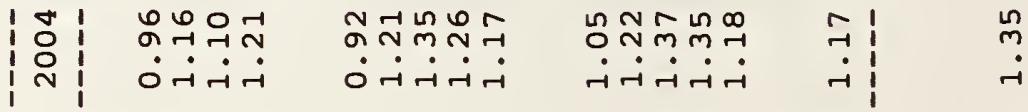

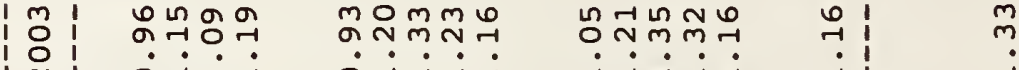

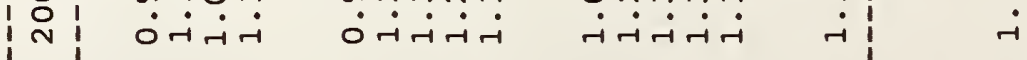

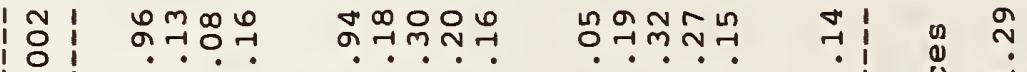

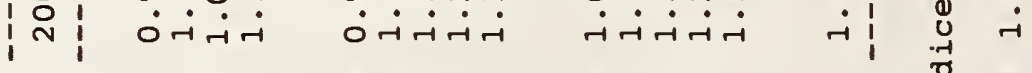

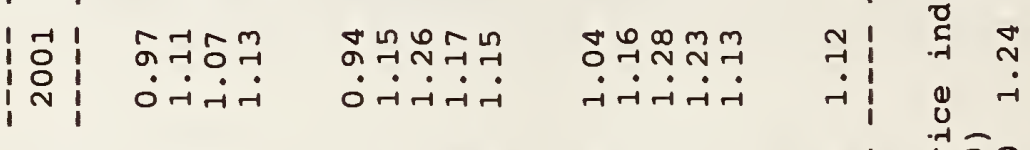

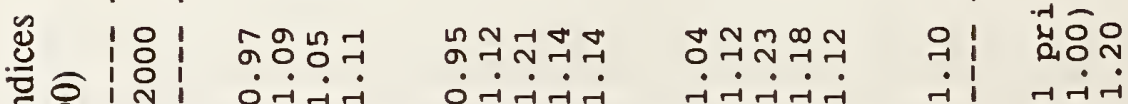

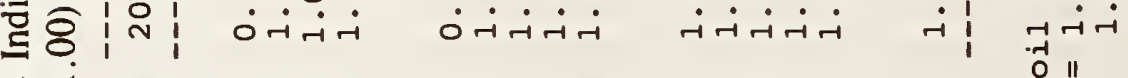

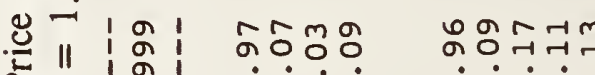

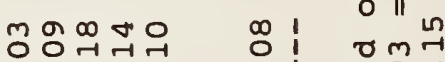

नंनंनंन मा चूरूे

पू山

至

Оंनंम ठनंनंन

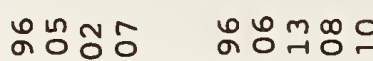

ผ

0.9470

:

๘

范范落

वेंभi

वेनंनंनं

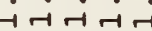

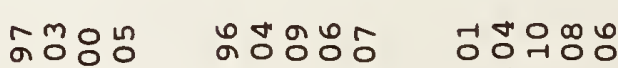

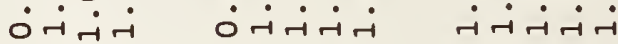

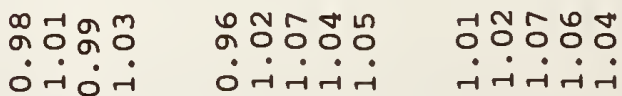

$+$

०艹

i.

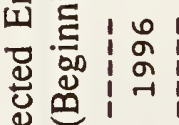

|

क००m रुगिm

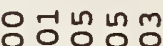

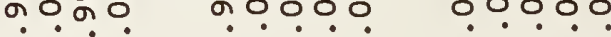

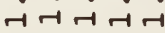

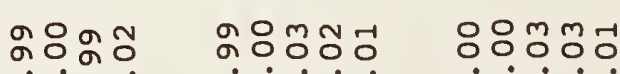

告

98000

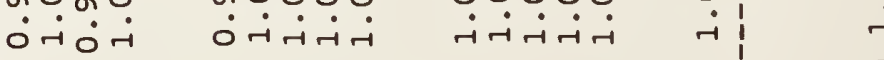

- \begin{tabular}{l|ll}
0 & 0 \\
\hline & 0 & 0
\end{tabular}

की के

길

न)

i)

:

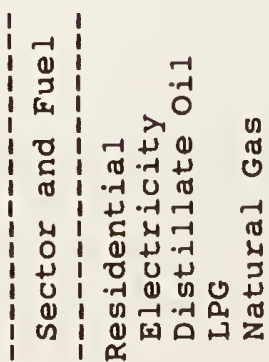

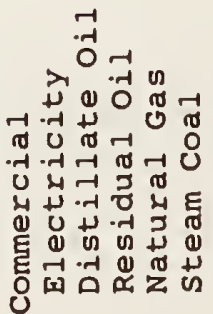

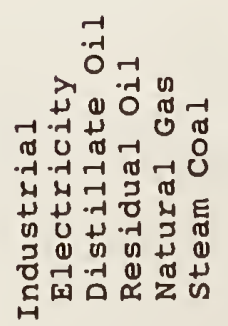

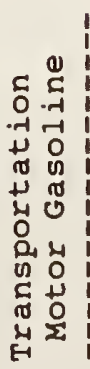

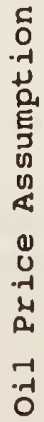




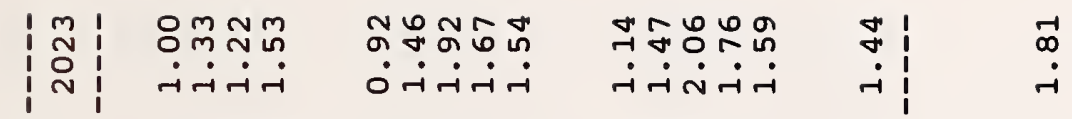

|

|

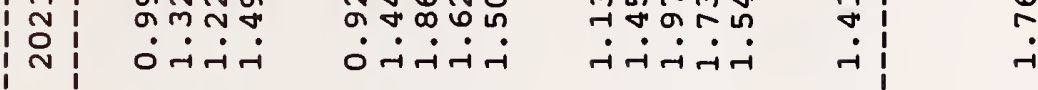

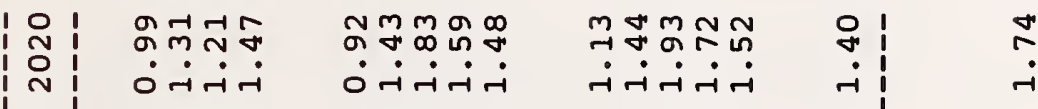

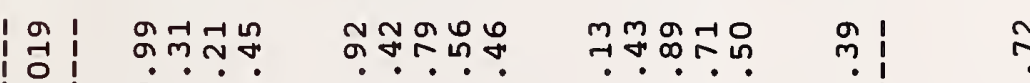

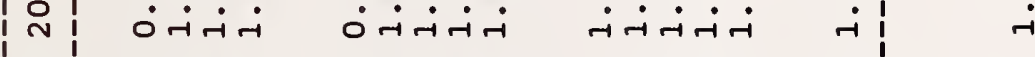

|

O

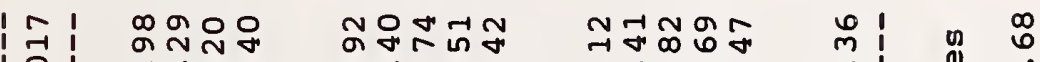

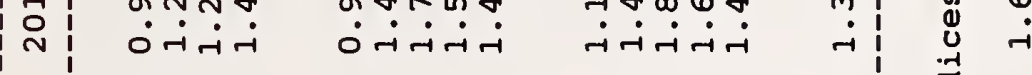

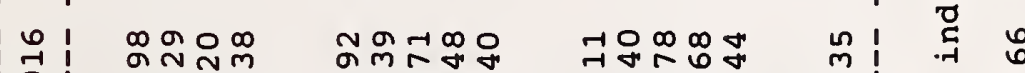

विनं

$\dot{0} \dot{0} \dot{0}$

नiनंनiनं

is -1

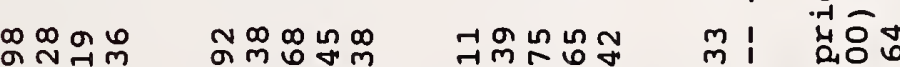

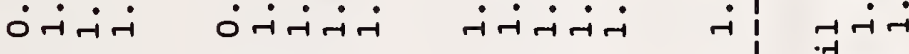

o

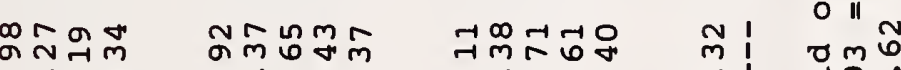

ठनंन ठनंनंन नंनंनं

ठननનन ननનनન

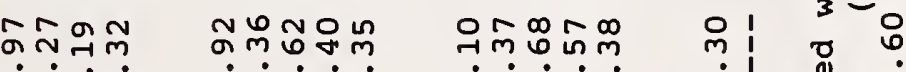

突

$\dot{0} \cdot \dot{\theta}$

$0 \cdot \dot{0}$

$0 न-1-1$

$0-\mu-1-1$

-1 -

눙요

Noon

or $\forall m o$

$\rightarrow$

齿

$\dot{0} \dot{-1} \dot{-1}$

- $0 . \dot{1}$

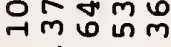

ค) $\stackrel{\oplus}{\sim}$ in

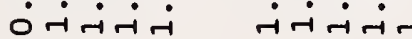

\begin{tabular}{l|ll}
$-i$ & 0 & n \\
-1 & के
\end{tabular}

ठ্ّ

in

N $\operatorname{nn}$ ก

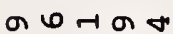

$\infty 1 \quad \infty$

$\dot{0} \dot{-1} \dot{-1}$

$\dot{0} \dot{-1} \dot{-1}-\dot{ }$

न-1न

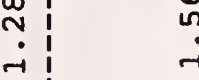

$\mid \begin{gathered}0 \\ -1 \\ 0 \\ 0\end{gathered}$

$\sin$

표잉

on in $\infty$ in $m$

०ंनंनंनं

-iनं-i

वनंनं

$-10-1-1$

$\infty m+m-1$

照

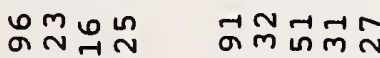

ठनंनंनं

om

ठनंनं

न-1-1

ฮ્ّ

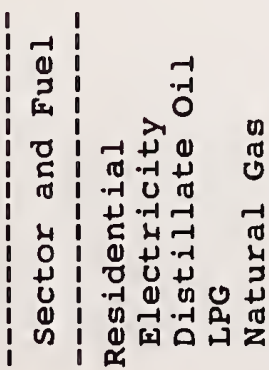

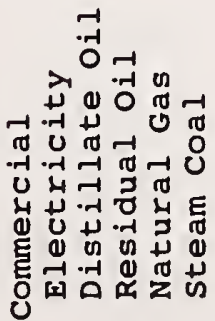

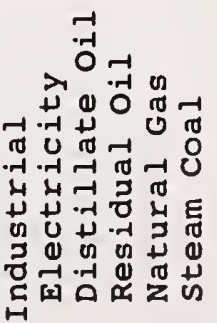

.

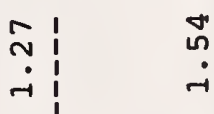




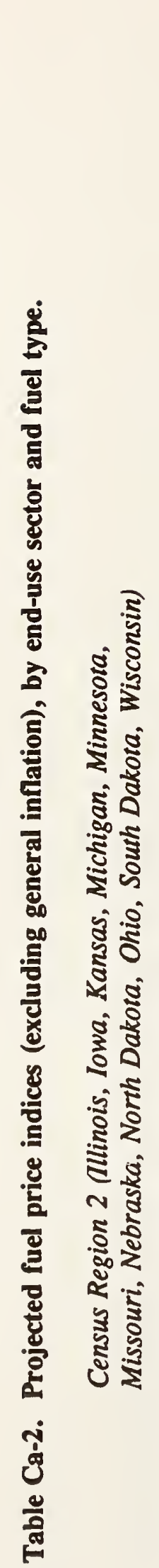

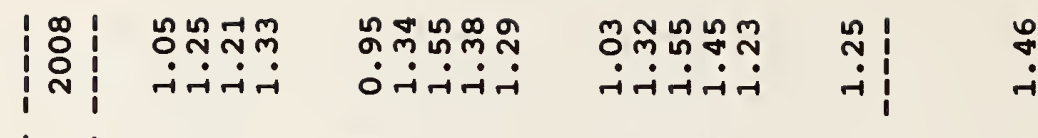

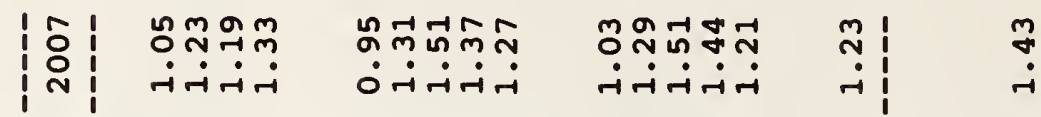

|

| 1 nowo

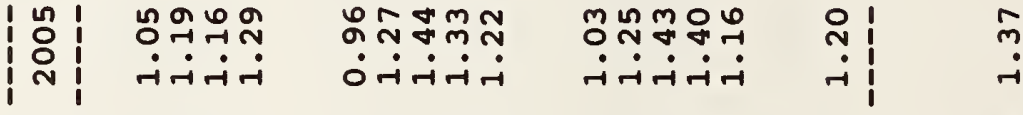

:

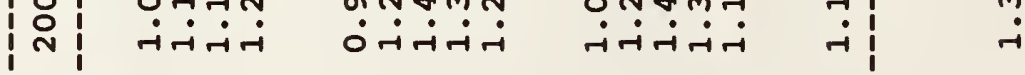

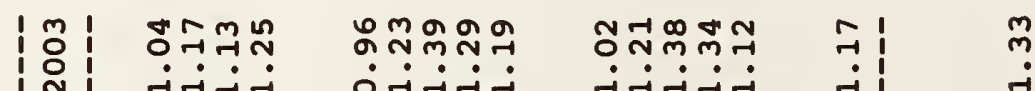

|

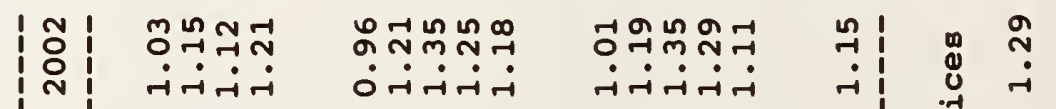

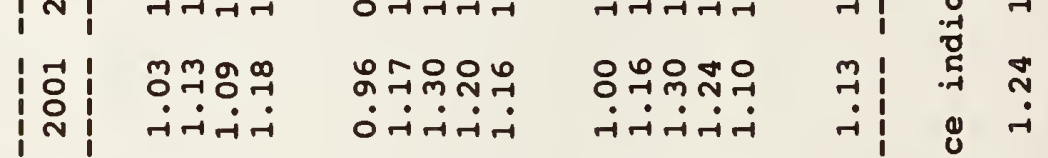

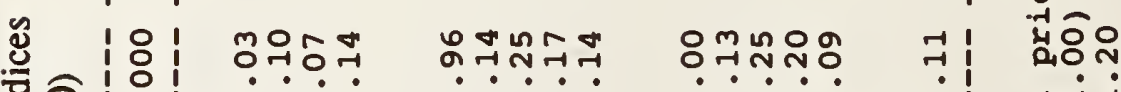

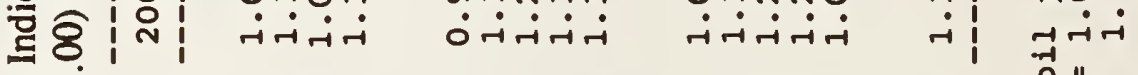

\&

ब्र

פ

ลำำำำ

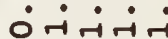

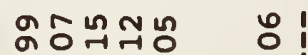

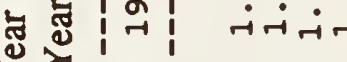

2)

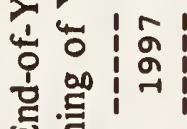

ㅇํㅇํำ

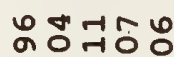

०ंनंनंने

نं $\dot{0} \dot{-1}$

Ð艹

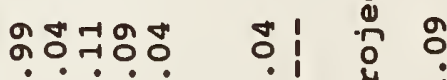

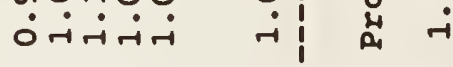

㟧

कूष

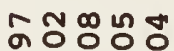

ठंनंनंन

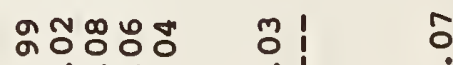

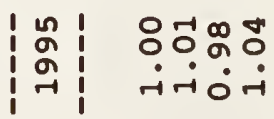

๓

ठंनिंन

ठनंतनंत

i

เป็

क ! मंत्म

ภำก

ด्

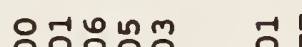

문

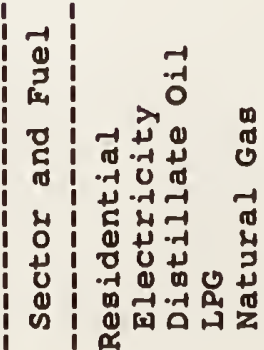

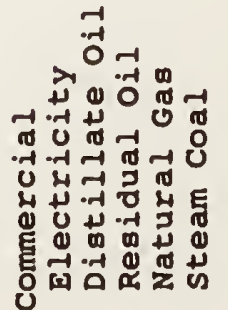

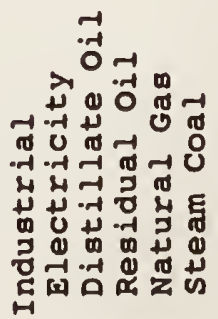

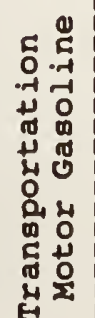

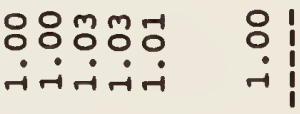

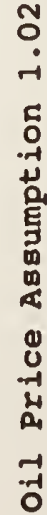




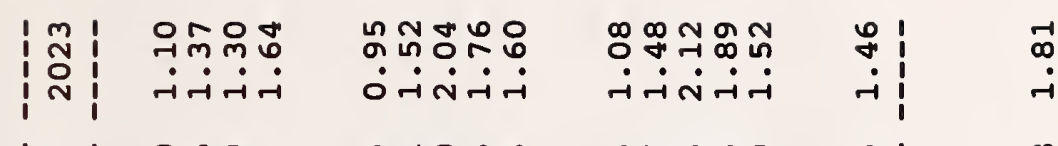

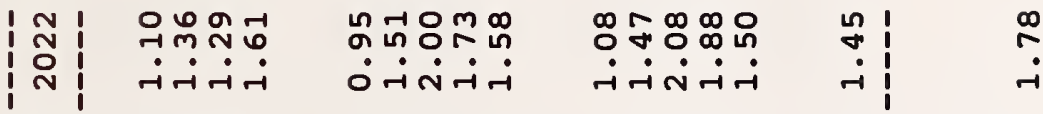

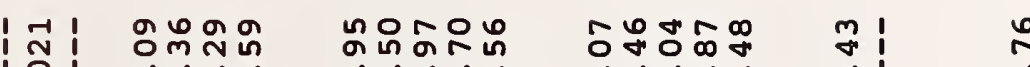

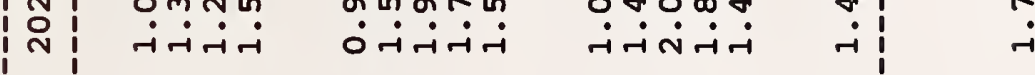

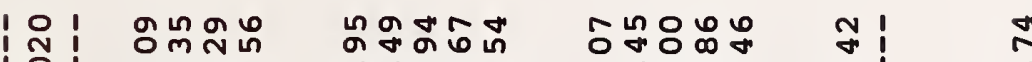

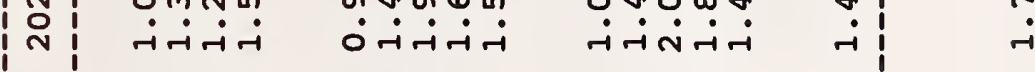

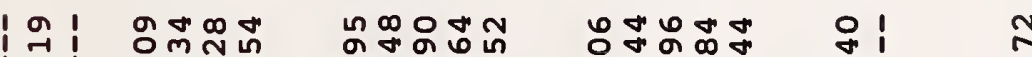

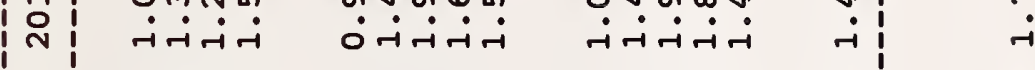

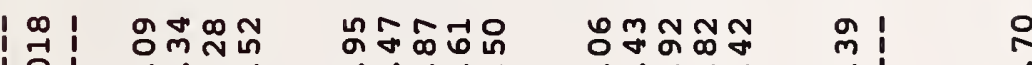

I नiनj

|

|

|

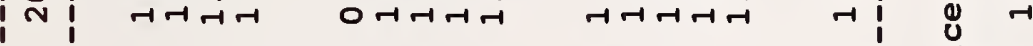

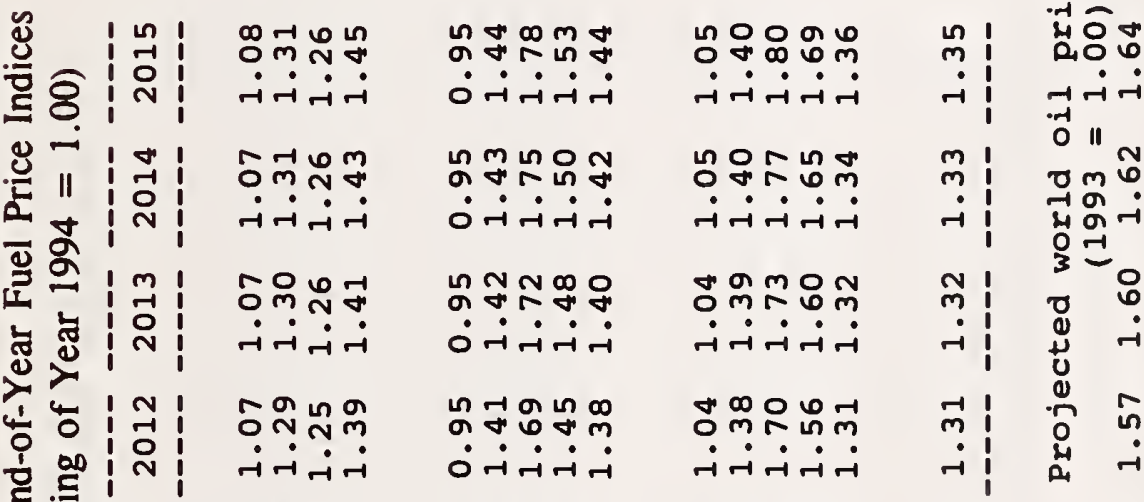

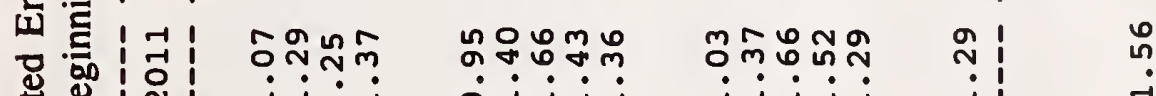

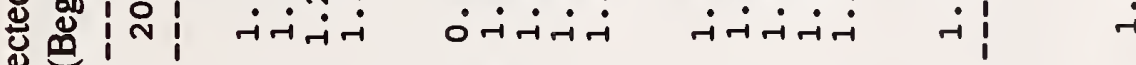

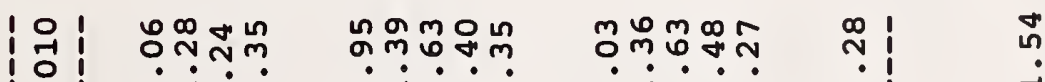

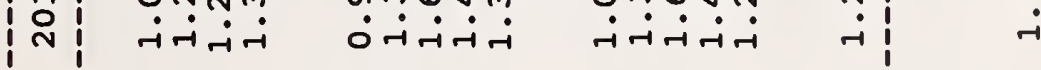

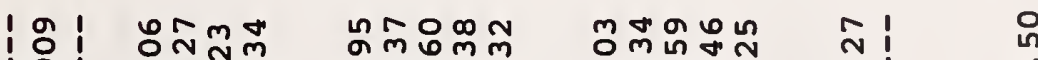

|

త્ટ

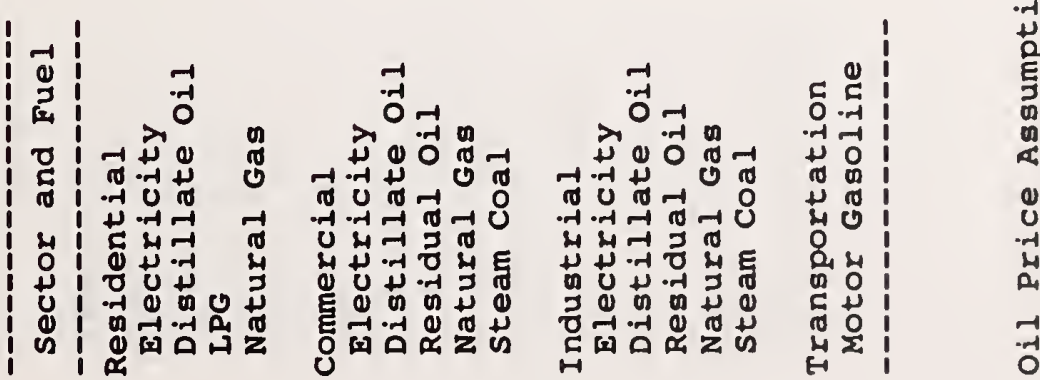




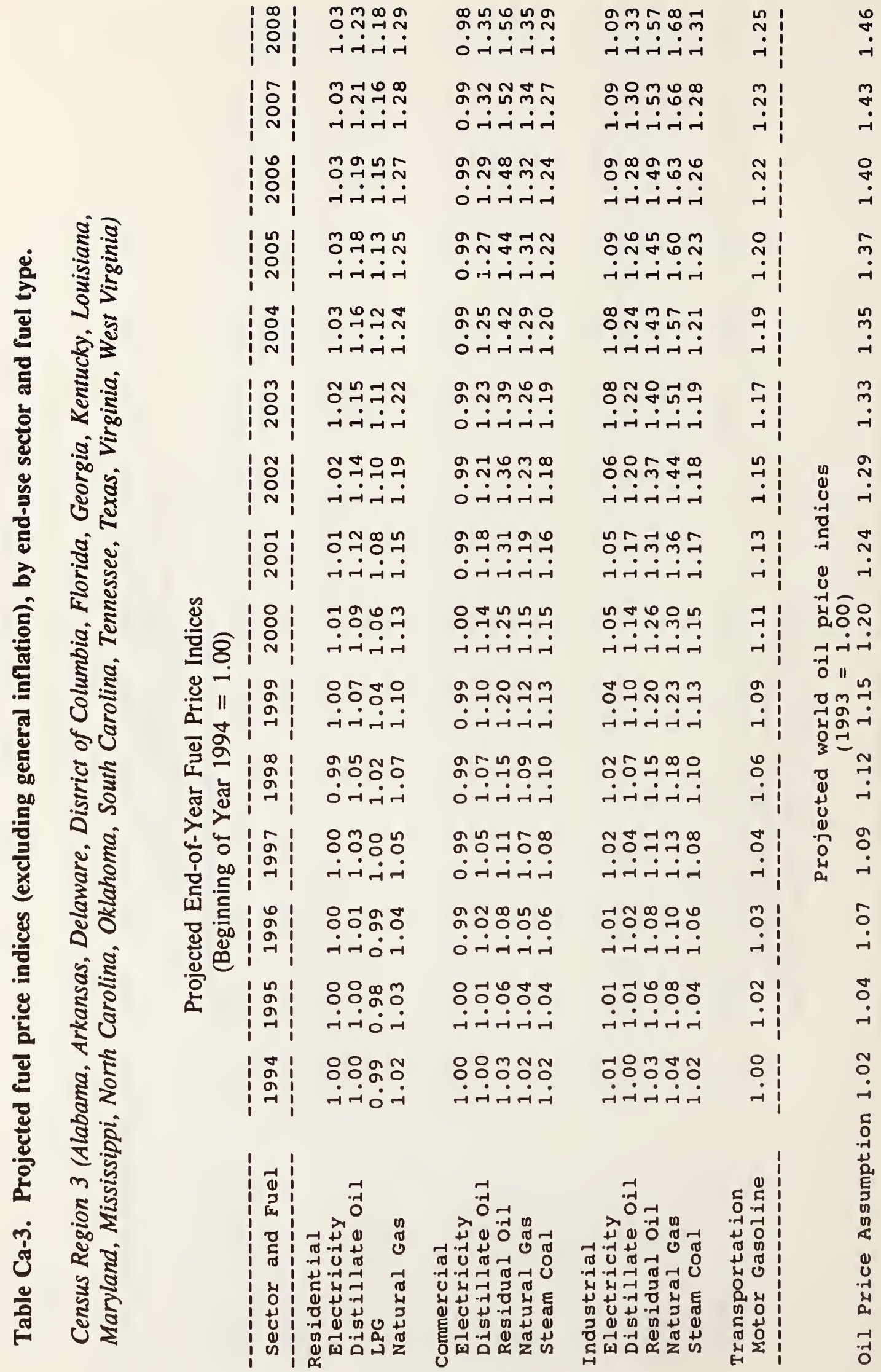


Im I

N

I त मतन म

N I

, 1 1 ?

न न म

ก I $0 m$ I

वे

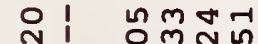

กิ

-iન-

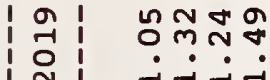

तi

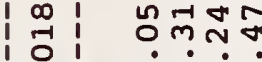

ता तो

吾|

नનन

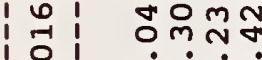

न-

ำกำำ

-iन-

เั $\stackrel{\infty}{\sim} \underset{\sim}{m}$

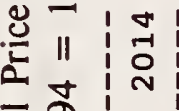

ฮू

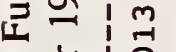

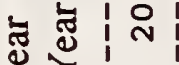
$\succsim$

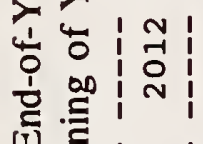

四

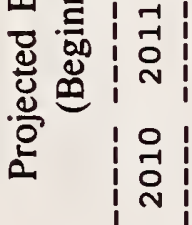

$\mid \begin{gathered}0 \\ \stackrel{2}{0} \\ \stackrel{2}{N}\end{gathered}$

| 옹

-iن

วัฒ $\stackrel{\infty}{\sim} \underset{m}{m}$

$\dot{-i}-\dot{-1}$

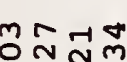

-iनं

mํำ

‥ก?

नंन्नान

ํํ융유

नiनं

ํำ

-iनं

नान्त

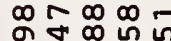

نंनंत्रि

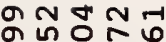

요의

$\approx$

oriñ

नंत्लं

ดิํํ덩ㅇำ

orini-i

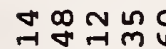

응용ํํ

-iंi

ठंनंनं

ホヘヘ

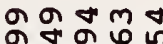

वंन्न-ं

オัง n

नांतें

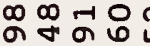

نं $\dot{-1}$

*กmm

-ंنं

\begin{tabular}{l|l}
-1 & $\infty$ \\
- & -1
\end{tabular}

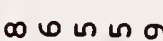

ดे

$\infty \ln \pi$

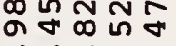

ठनंनं

mo둥

-iनंत्रिं

$\stackrel{n}{*}$ ?

$\infty \pi a$

。ํํ용

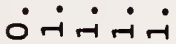

$m$ ำก넌

तंत्रिं

$+1$

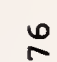

-

mon

䑻웡

ठनंनंनं

궗ㅇㅇㅇㅇㅇ

नंत्नंत्

ริ

$-1$

니

\section{काma}

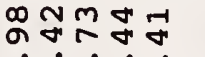

0ंनंन्म

พู่ากร

नंत्रंत्रं

พำตำ

-iri-i

m) ำ

Drona

ᄀำ

नंनंत्नं

$\infty$ I

$\begin{array}{ll}n & \infty \\ 0 & \vdots \\ 0 & -1 \\ 07 & -1 \\ 0 & 0\end{array}$

小的m

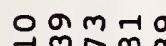

0ंनंनं

$\dot{i} \dot{-i} \dot{-i} \dot{-i}$

웅ㅎํ요

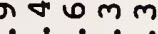

잉요

$\dot{0} \dot{-1} \dot{-1}$

-i-i

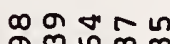

भ̆ต?

O

-i-i-i

文

के I

-i

今 7

n !

붕용

०-

Drom

ติตั

ํำำ

$-i$

ชิ

ชิ

मून न

\begin{tabular}{l|l}
$m$ & $3-0$
\end{tabular}

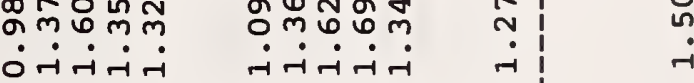

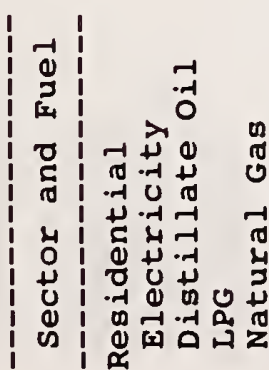

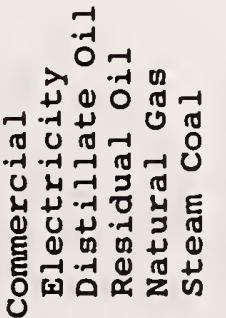

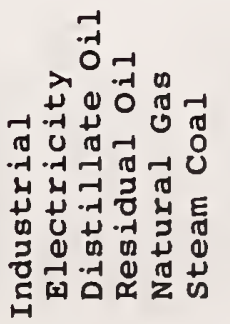

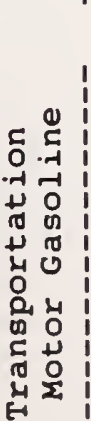

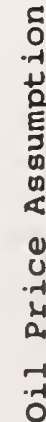




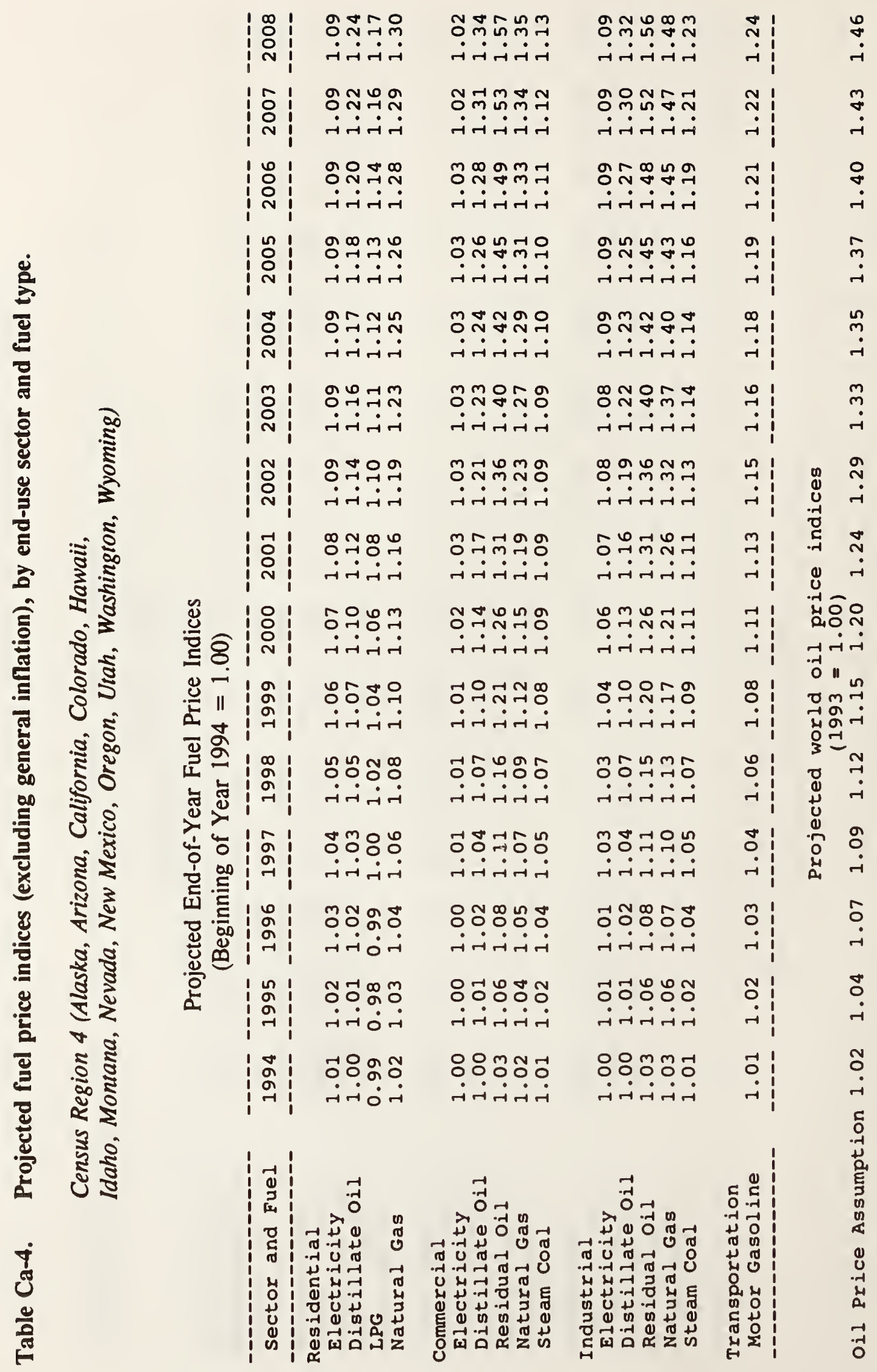




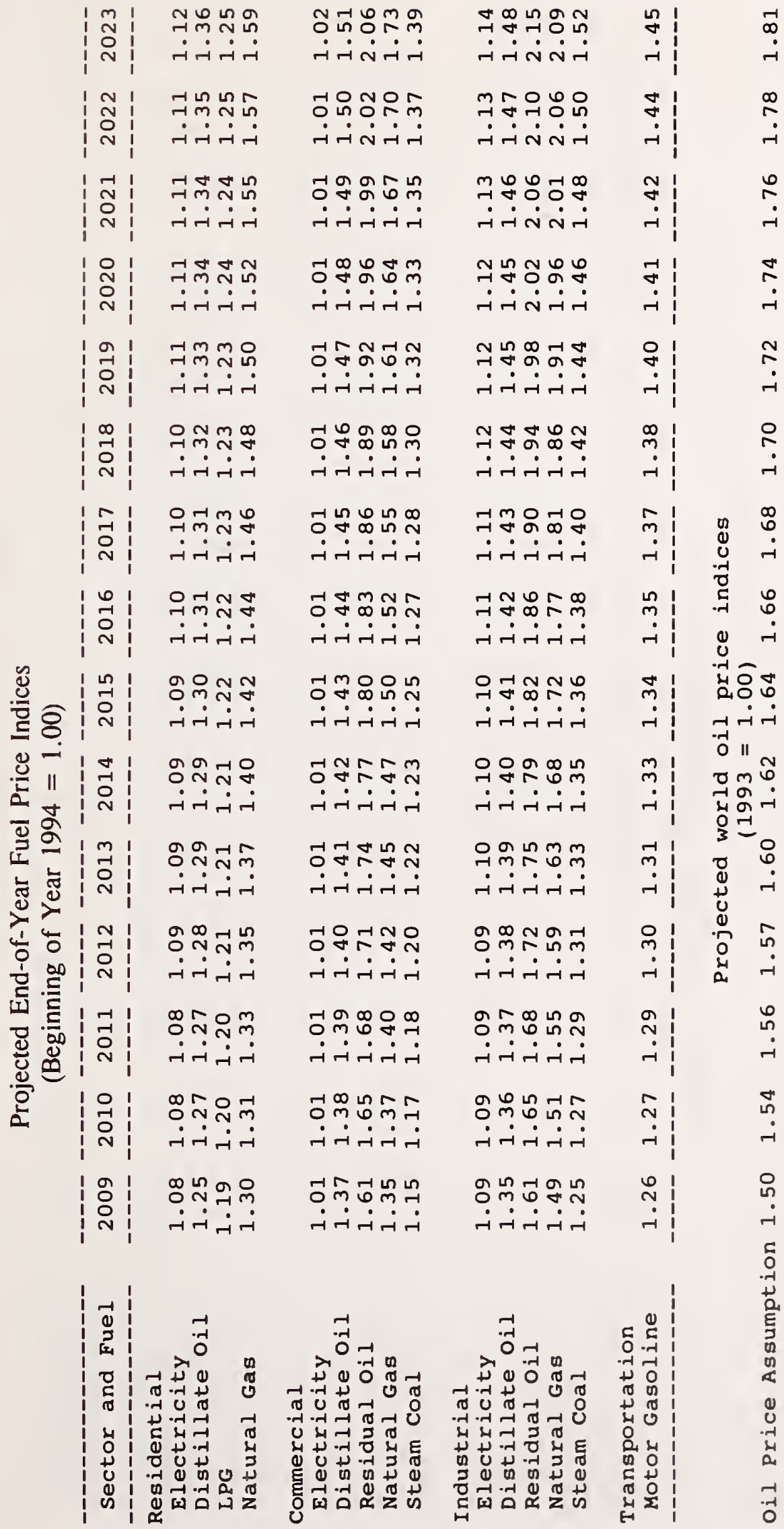




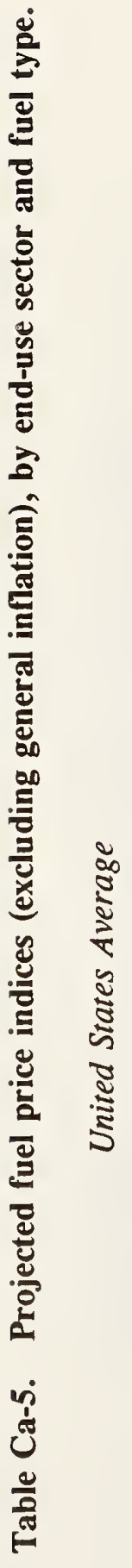

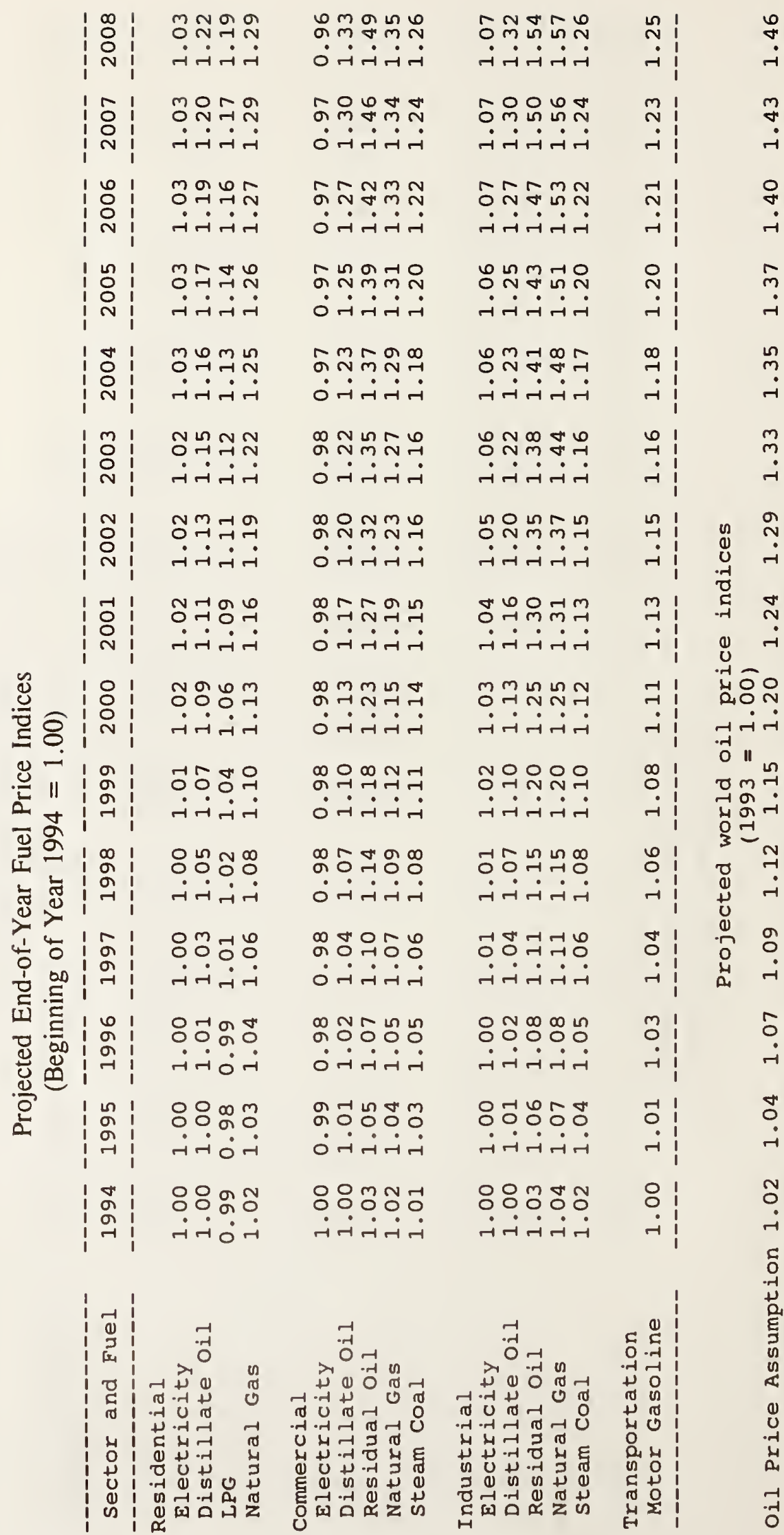




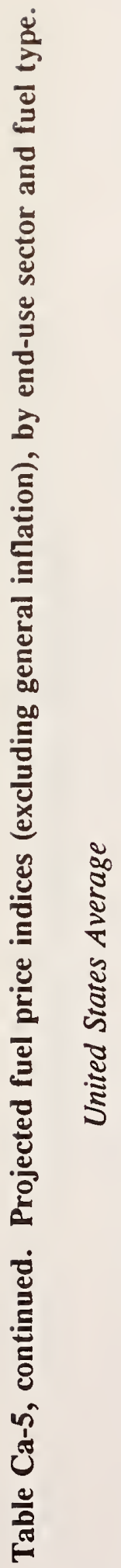

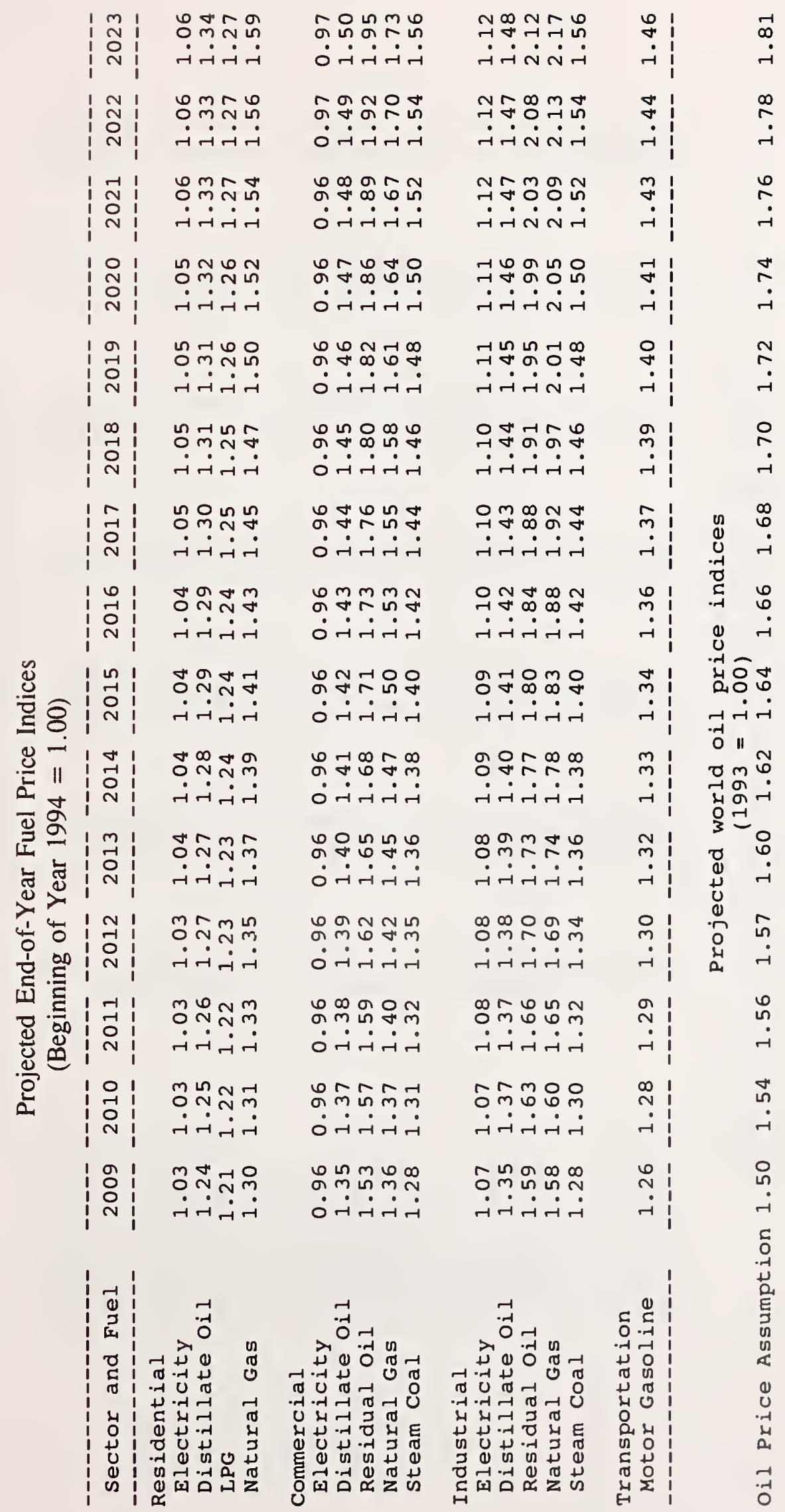




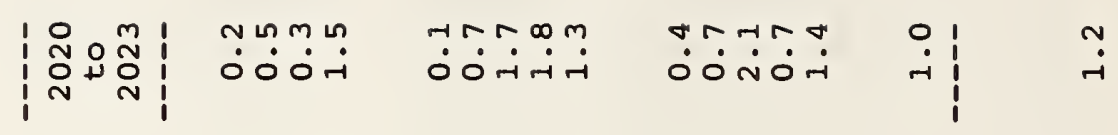

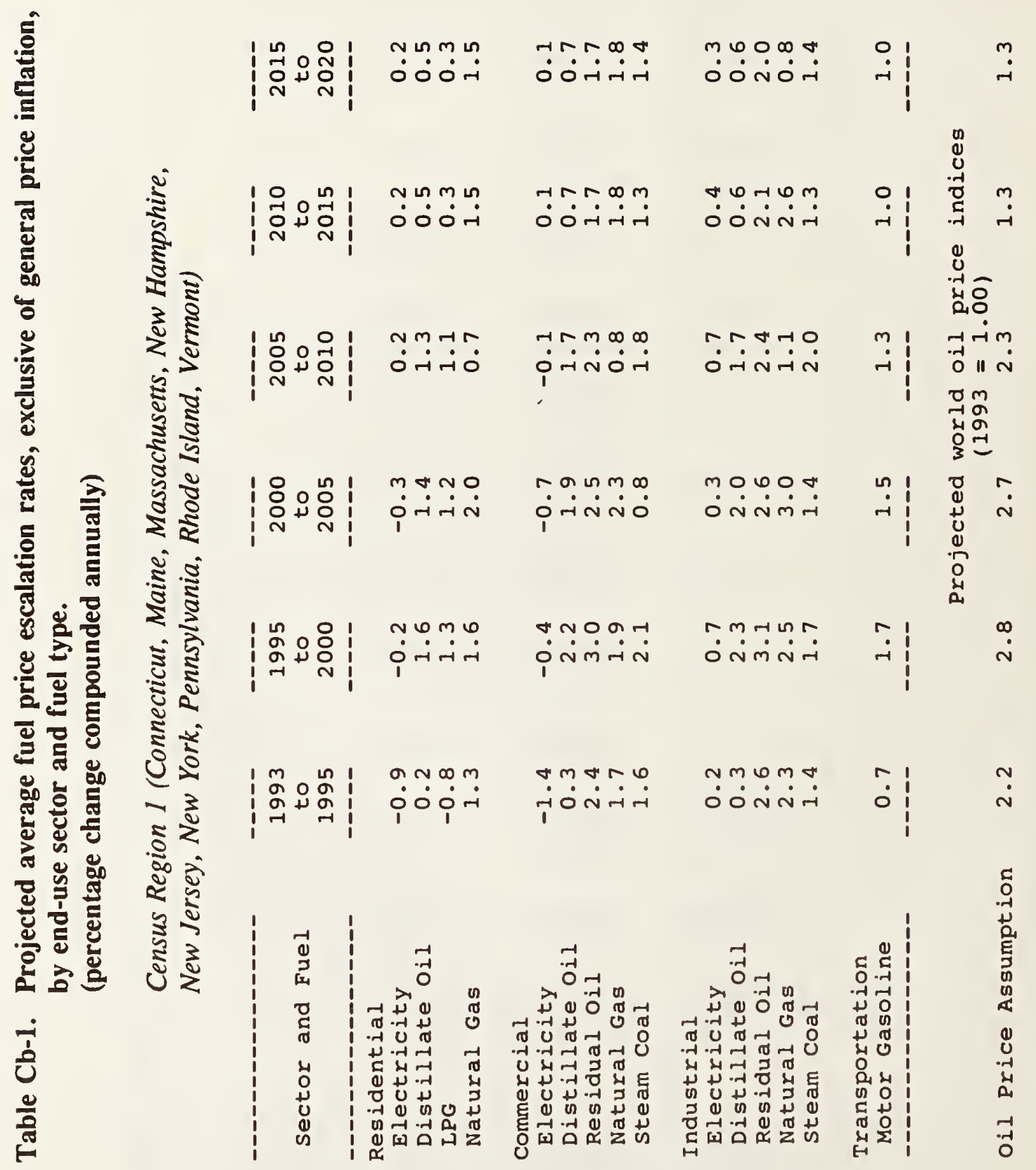




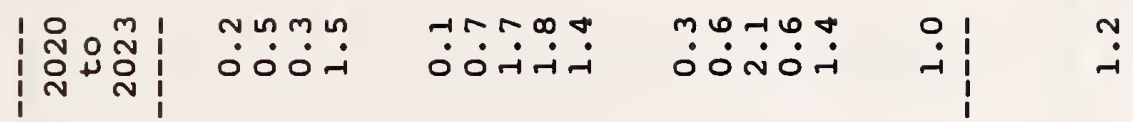

|

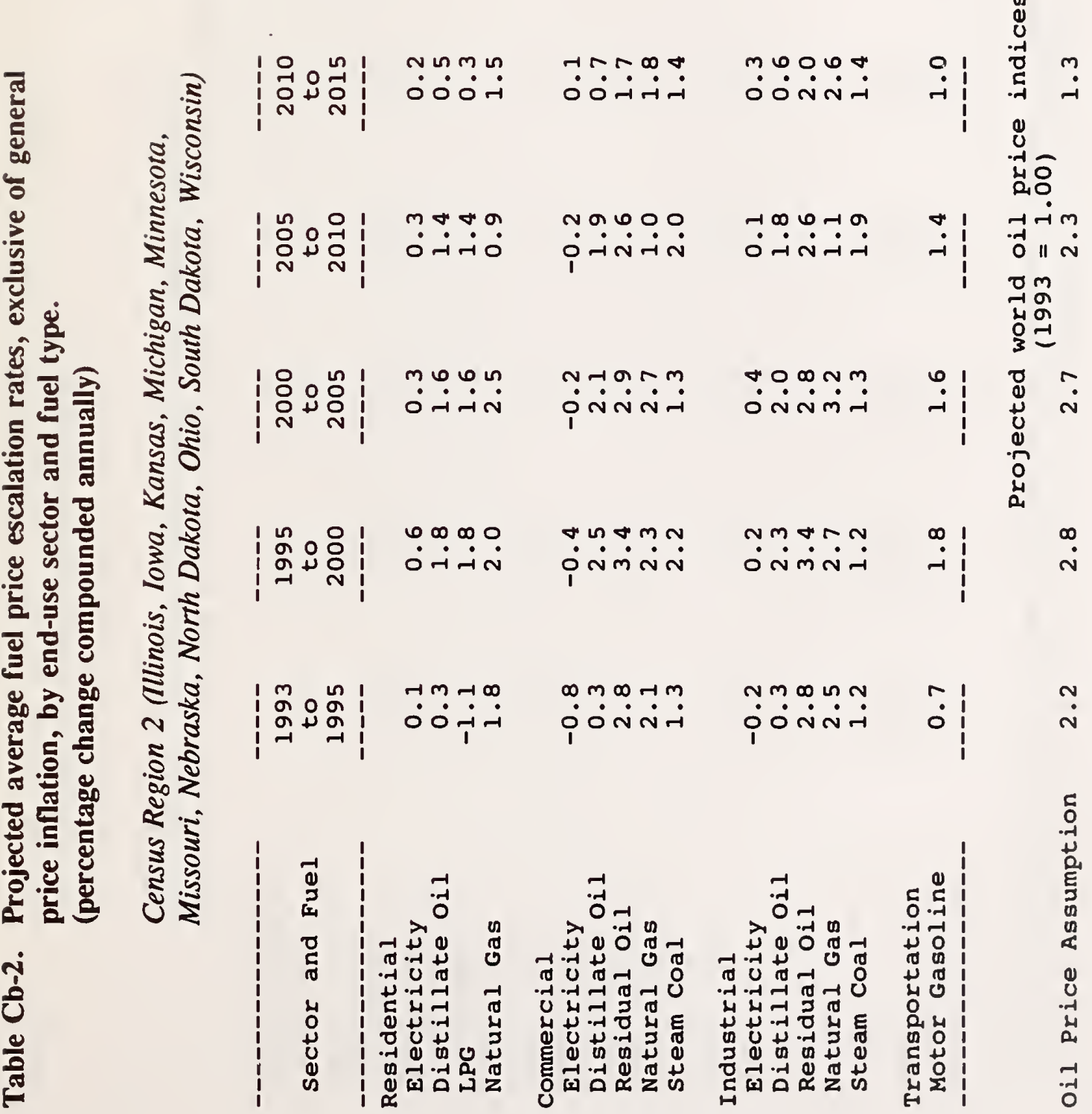




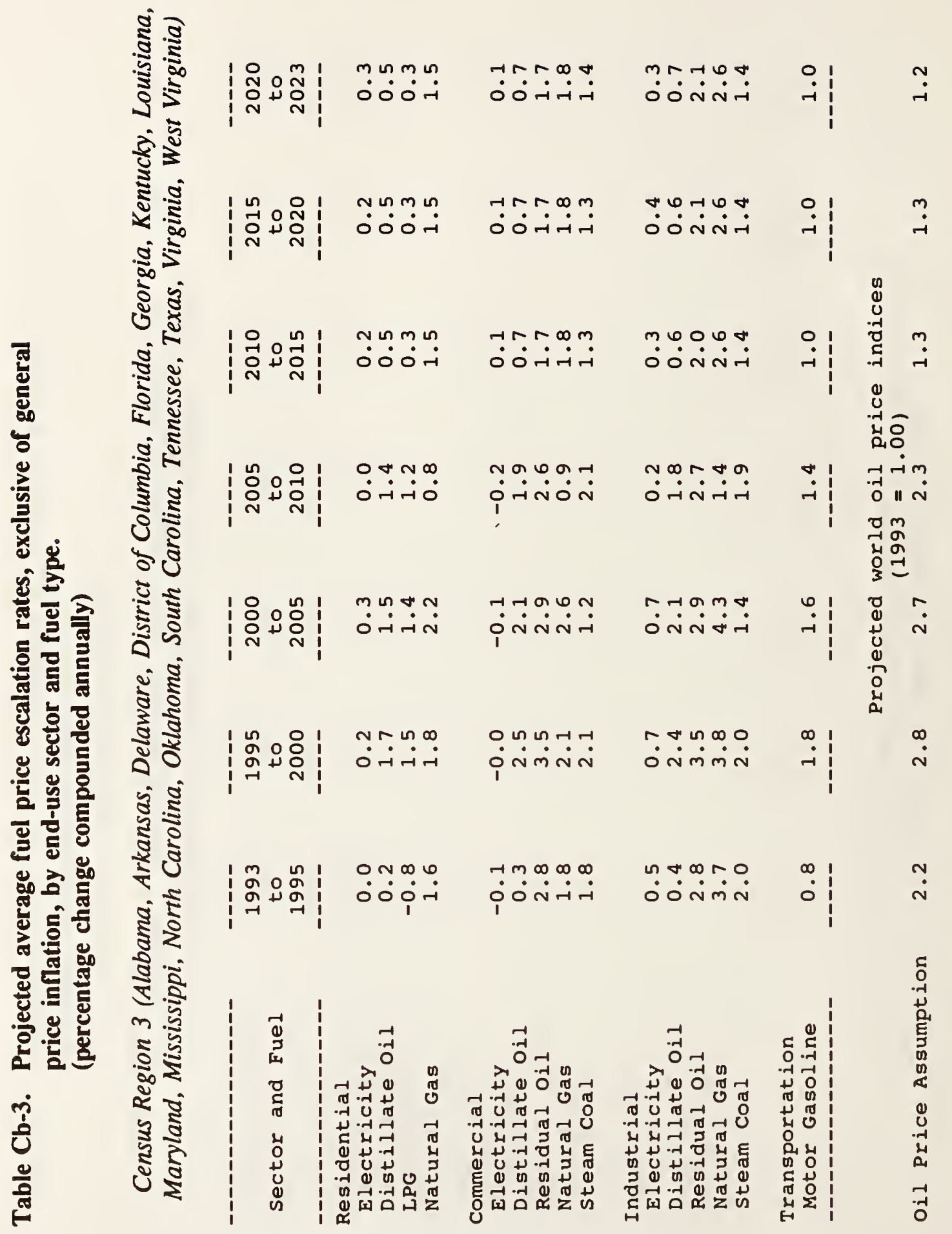




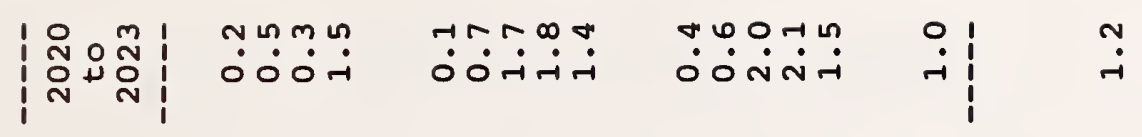

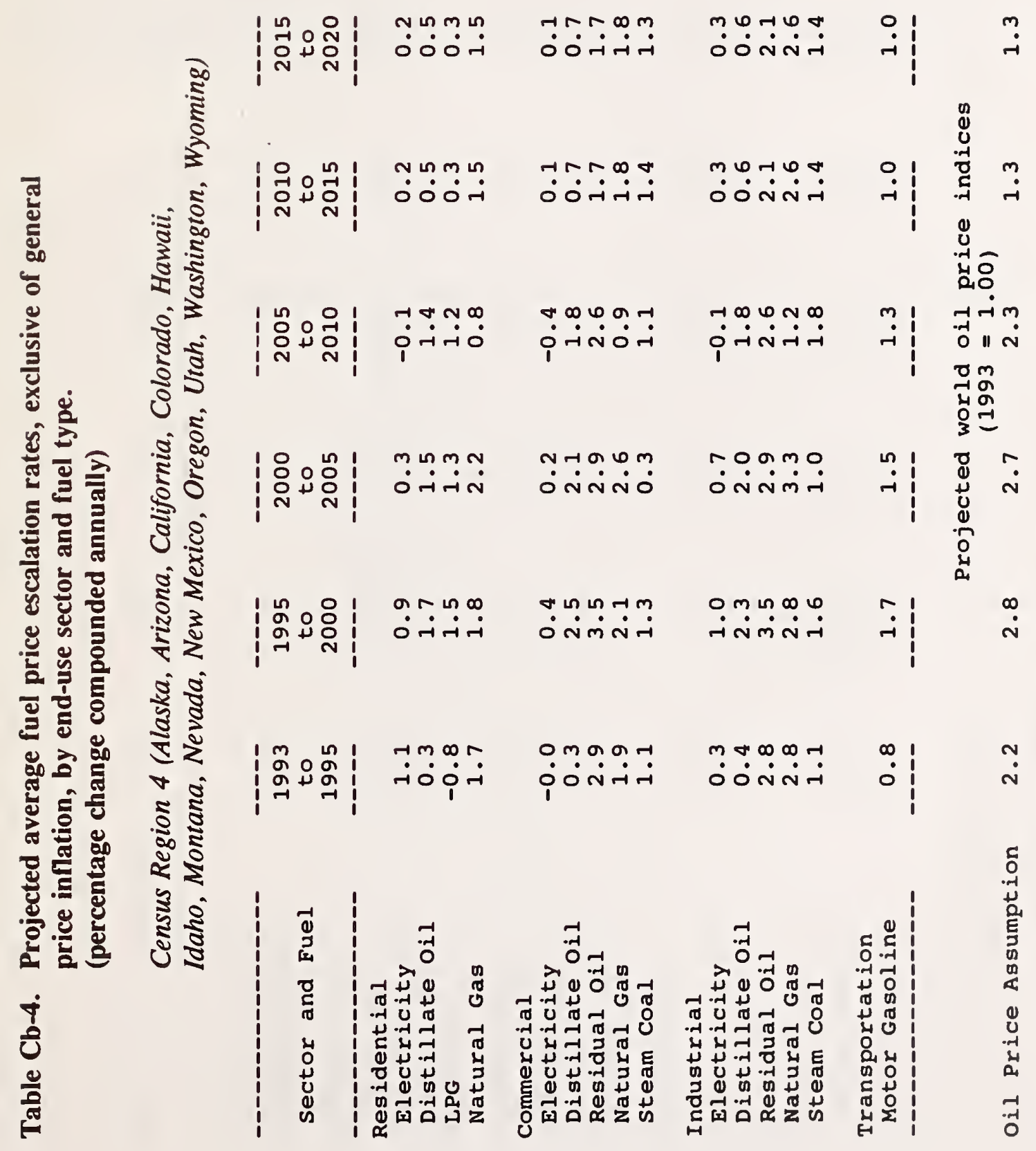




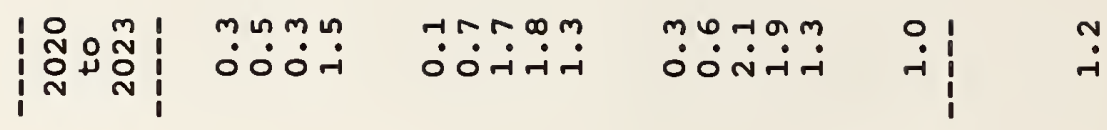

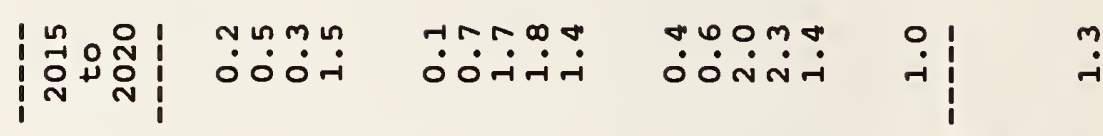

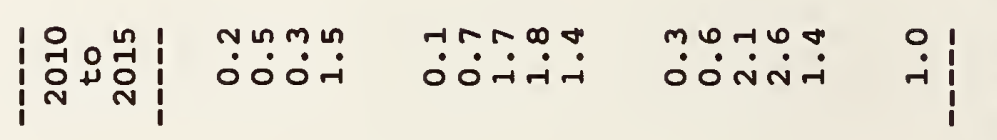

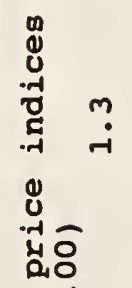

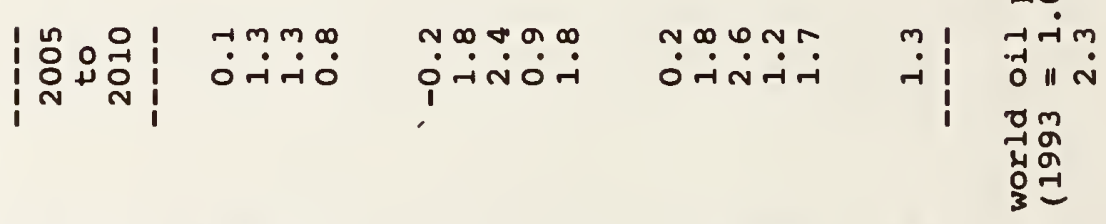

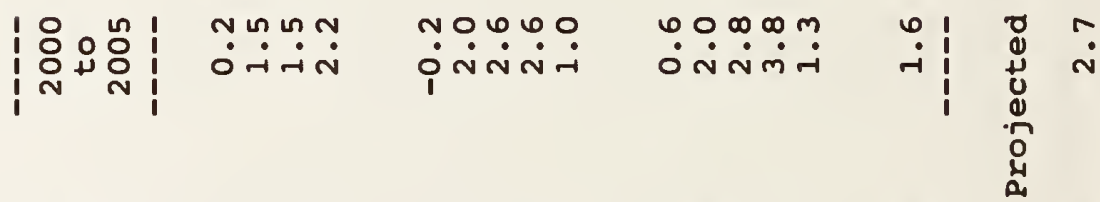

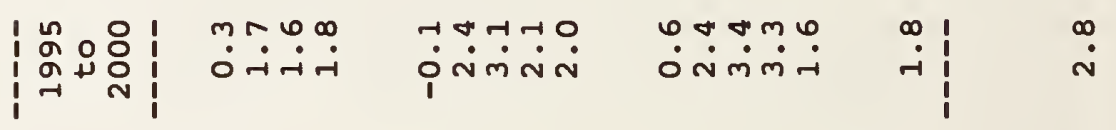

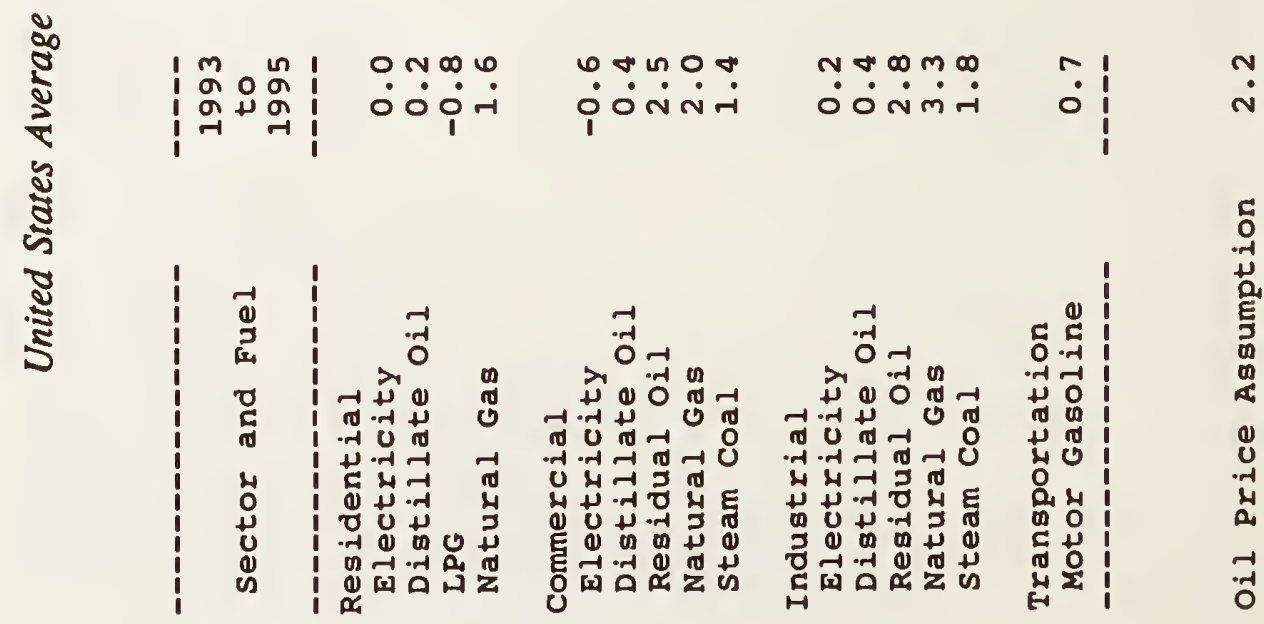

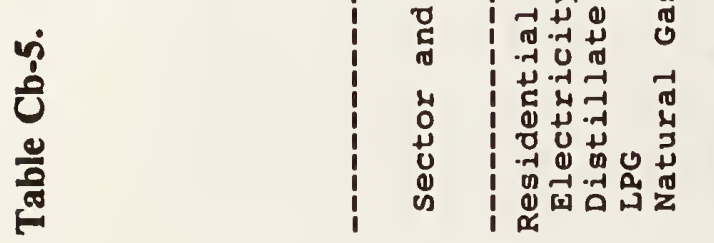


D. Factors for Updating Appliance Label Values in Compliance With the Energy Conservation Mandatory Performance Standards for New Federal Residential Buildings (10 CFR 435)

Compliance with energy conservation performance standards for new Federal residential buildings requires calculation of a building's energy costs, including appliance costs. For this purpose, label values for gas and electric water heaters are given in the Federal micro-computer program, COSTSAFR, as $\$ 176$ and $\$ 406$ per year, respectively, in 1987 dollars, and for refrigerator/freezers, as $\$ 61$ per year in 1987 dollars. To adjust 1987 prices to 1993 prices, multiply these 1987 label values by the factors below.

Table D. Factors for updating appliance label values from 1987-1993 dollars.

\begin{tabular}{lc}
\multicolumn{1}{c}{ Fuel } & Factor \\
\hdashline Gas & 1.17 \\
Electricity & 1.16
\end{tabular}




\section{PART II: TABLES FOR PRIVATE SECTOR LIFE-CYCLE COST ANALYSIS}

Projected average fuel price indices for private sector use (Indices are given inclusive of four alternative rates of general price inflation)

This section presents in Tables S-1 through S-5 projected average fuel price indices for 4 fuels in the residential sector and 5 fuels in the commercial sector for each of the years from 1994 through 2023. They update tables originally published in the report, Comprehensive Guide for Least-Cost Energy Decisions (NBS SP 709).

As a convenience for the user, the indices include the effect of four alternative, hypothetical rates of general price inflation: $3,4,5$, and $6 \%$. Selection of these rates is intended in no way to suggest what actual rates might be. Use of the indices produce price estimates which are in current dollars, inclusive of general price inflation. Current dollar prices are needed when discounting is performed with discount rates which include general price inflation.

The indices based on inflation rates of $3,4,5$, and $6 \%$ allow the analyst to perform evaluations based on the assumption of a positive rate of general price inflation that changes the purchasing power of the dollar. Performing evaluations in current dollars is sometimes preferred for private investment decisions, primarily because it facilitates the treatment of taxes.

The indices in Tables S-1 through S-5 are derived from the indices reported in Tables Ca-1 through $\mathrm{Ca}-5$ by means of the following equation:

$$
I_{S}=I_{C} x(1+g)^{N},
$$

where $I_{S}=$ index found in Tables S-1 through S-5;

$\mathrm{I}_{\mathrm{C}}=$ index found in Tables $\mathrm{Ca}-1$ through $\mathrm{Ca}-5$;

$\mathrm{g}=$ annual rate of general price inflation in decimal form; and

$\mathbf{N}=$ number of periods, in this case equal to the year of the index minus 1993.

Example of How to Use the Indices:

Suppose you wish to estimate the present value of energy savings in year 2000, and you expect an annual inflation rate of $5 \%$ per year. Taking natural gas for residential use in Maryland, estimate present value savings as follows: (1) multiply today's price for residential natural gas in Maryland by the projected quantity of energy savings in the year 2000 to estimate savings in the year 2000 in today's prices; (2) go to Table S-3, find the year 2000 index for residential natural gas at a $5 \%$ inflation rate (1.58), and multiply the index by the result from (1) to determine savings in the year 2000 in 2000 prices; and (3) discount the savings back to the present, using an SPV factor based on a discount rate that reflects a $5 \%$ inflation rate. To obtain present value savings over the entire study period, this calculation must be repeated for each year that there are 
savings, and the results summed. (UPV factors are not given for private sector use because of the large number of tables required to cover potential discount rates that might be used by the analyst.) The BLCC computer program can perform LCC analyses using any inflation rate and discount rate, in constant or in current (market) dollars. The DISCOUNT program, included with BLCC, can compute UPV ${ }^{*}$ factors based on DOE energy price escalation rates using any inflation rate and discount rate. (See page iv for more information on these programs.) Of course the private sector analyst may use the UPV ${ }^{*}$ factors provided in Part I, provided the analysis is performed in constant dollars, and the desired discount rate is either $3.1 \%$ or $10 \%$. The DISCOUNT program (version 3.3) included with BLCC can compute UPV factors on a personal computer for any discount rate (see page iv for information on DISCOUNT).

For further explanation of the use of these indices, see NBS Special Publication 709, appendix B, Part I.

The data in the tables which follow are reported for the 4 Census regions and the U.S. average. Figure B-1 presents a map showing the states corresponding to the 4 Census regions. The Census regions do not include American Samoa, Canal Zone, Guam, Puerto Rico, Trust Territory of the Pacific Islands, or the Virgin Islands. Analysts of Federal projects in these areas should use data which are "reasonable under the circumstances," and may refer to the tables with U.S. average data for guidance. 


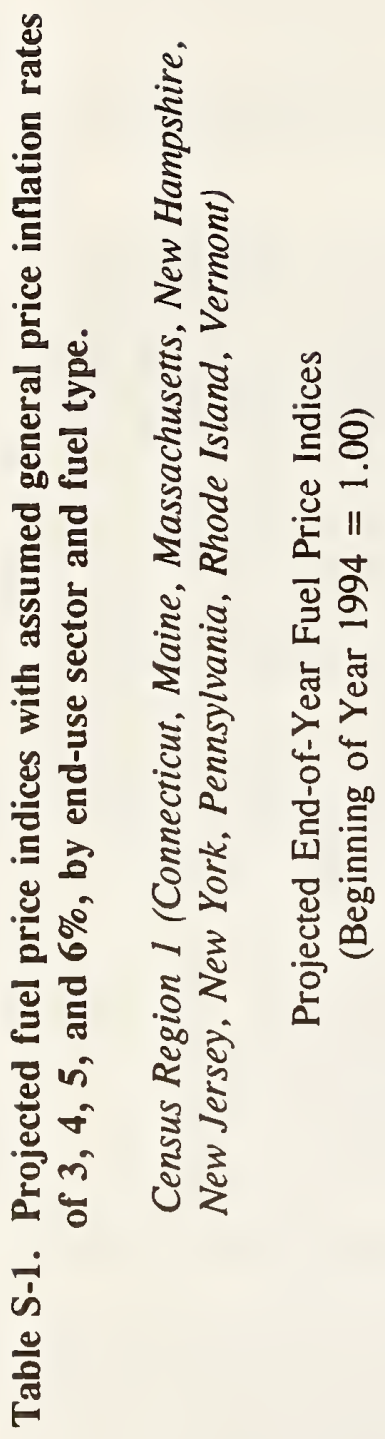

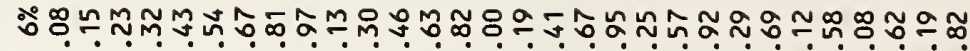
芯

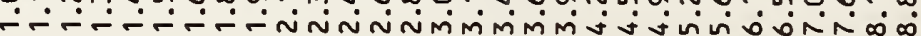

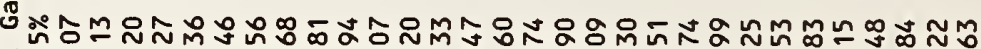

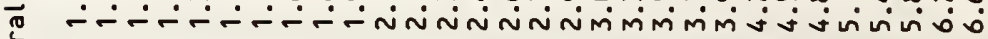

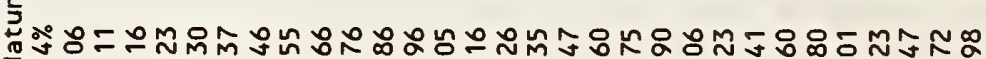
$\therefore \therefore-\therefore$ -

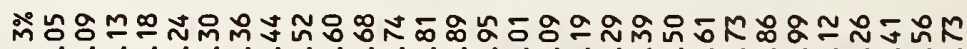
$\because \therefore \therefore ?$

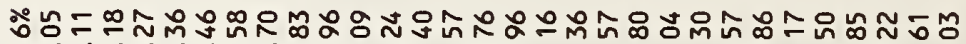

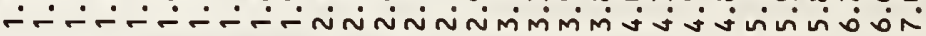

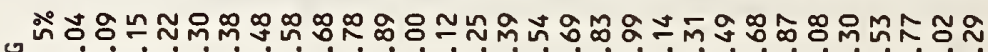

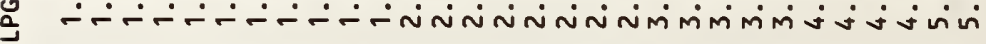
ช゚

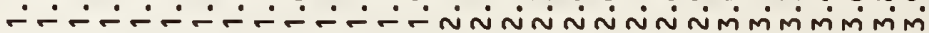

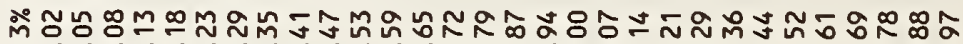

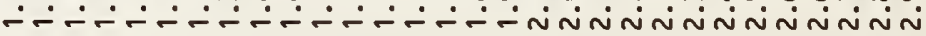

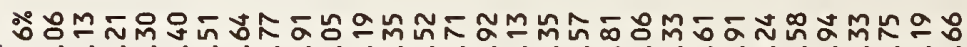

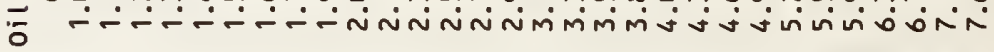

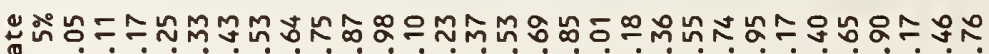

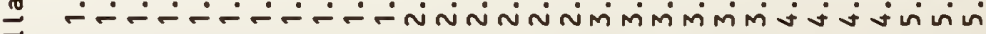

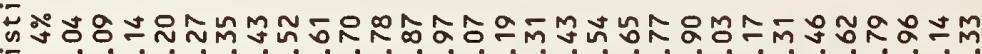

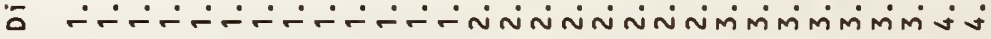

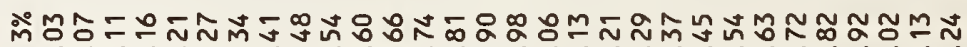

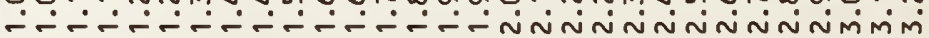

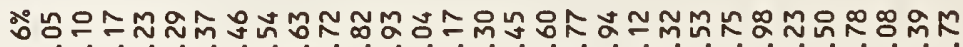

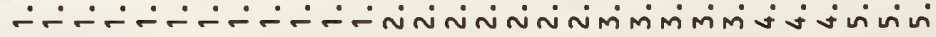

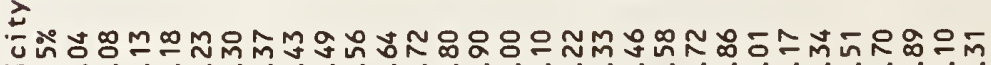

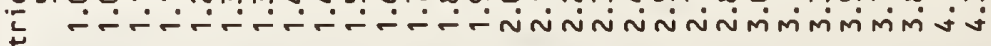

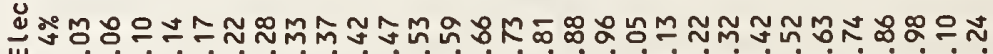
-

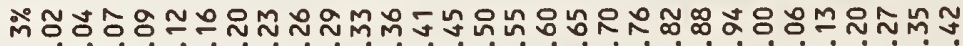

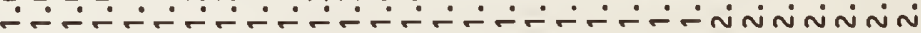

ฮัง 
ถำำำำษ

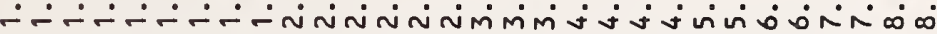

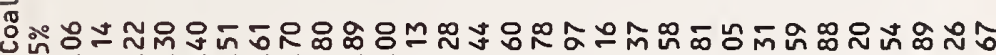

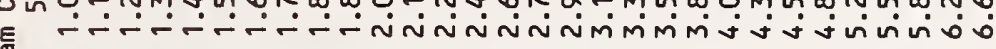

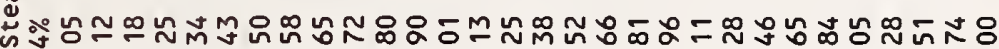
$\therefore-\therefore-1$ -

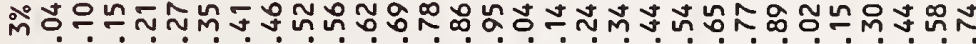

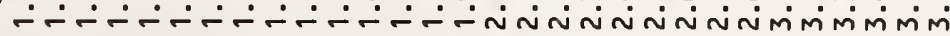

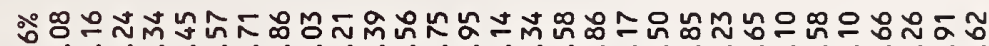
品

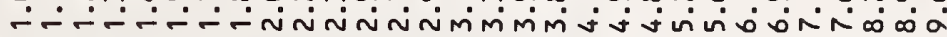

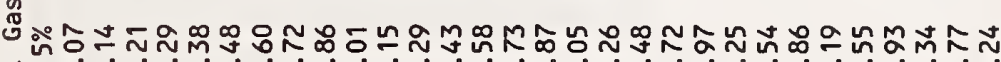

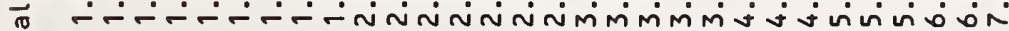

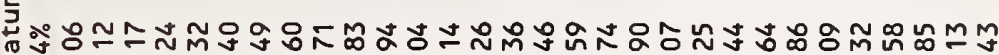

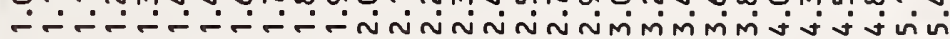

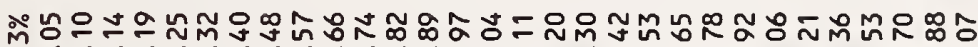

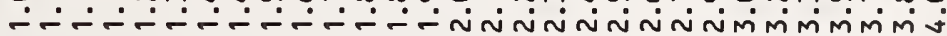

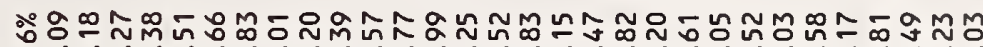
$=$

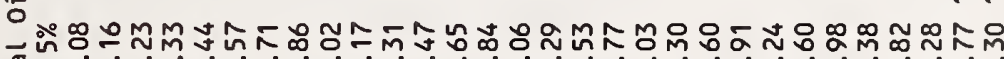

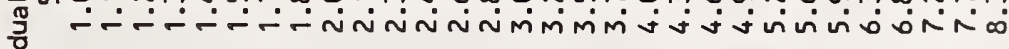

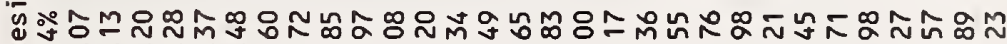

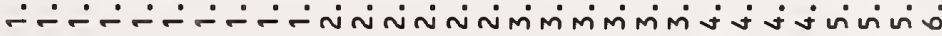

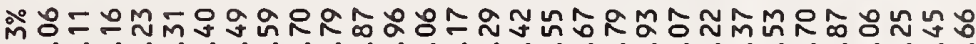
$\therefore \therefore-\therefore$ N $\therefore$ N

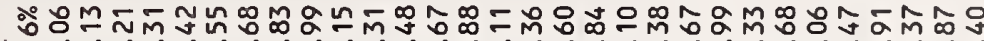
б -

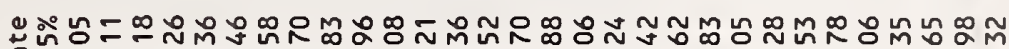

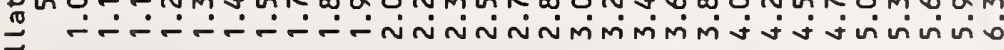

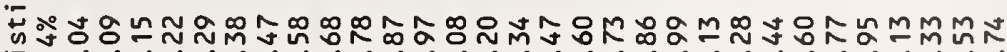

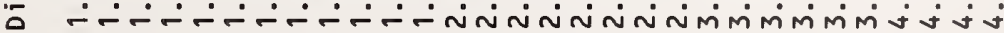

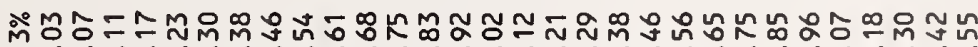
$\therefore-\therefore$ -

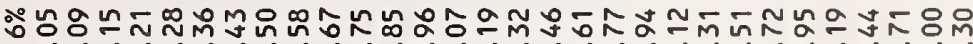

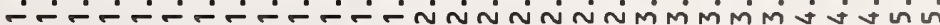
tิ

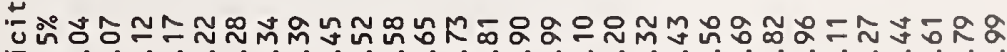

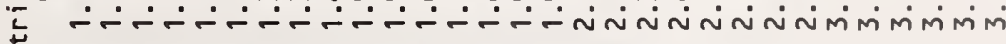

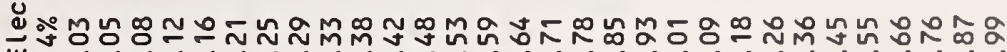

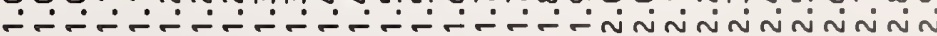

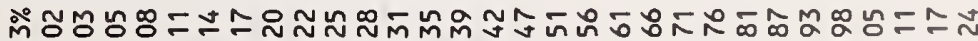

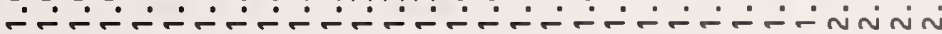

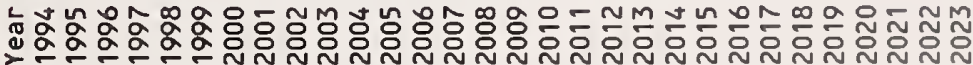




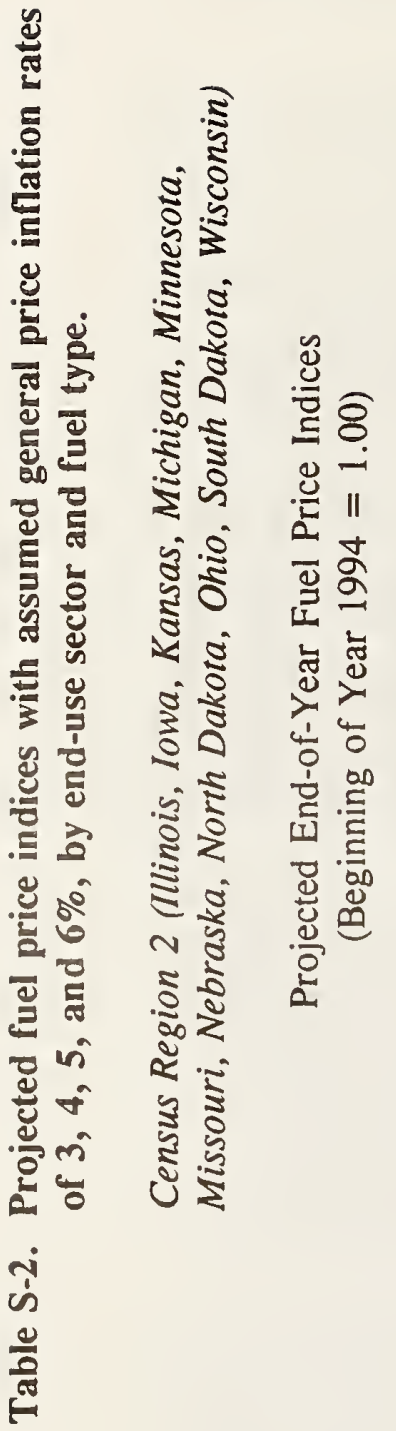

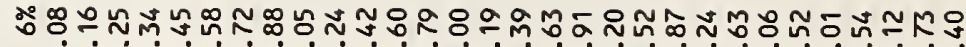
查

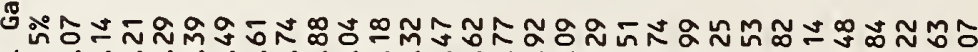

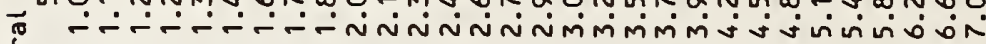

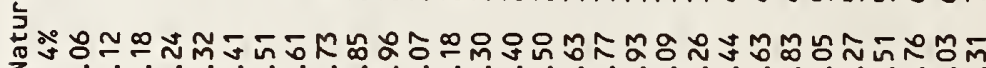

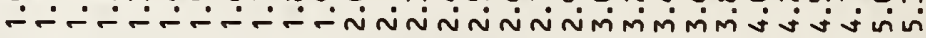

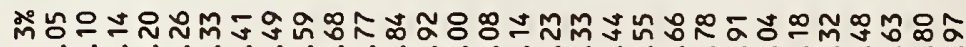

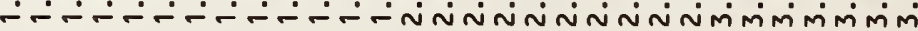

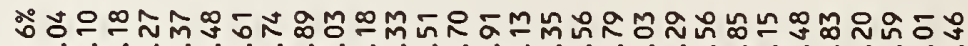

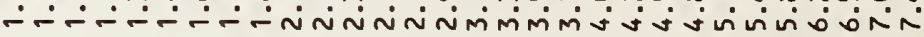

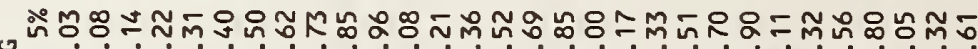

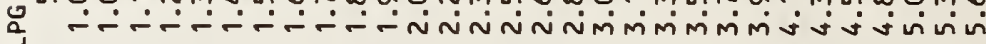

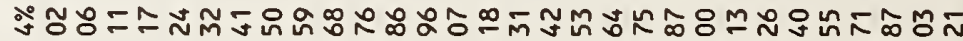
$\because \therefore \therefore-\therefore-\therefore$ iñunininimmimimis

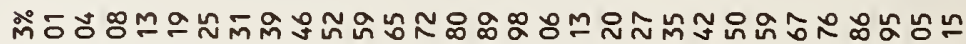

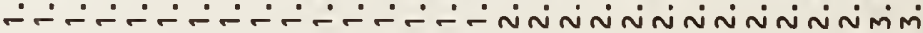

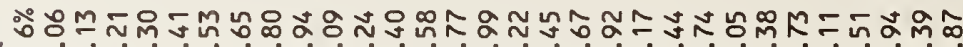
б - - - - N N

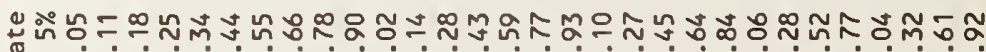

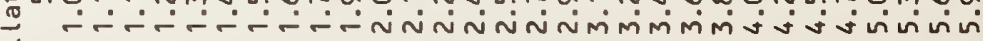

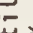

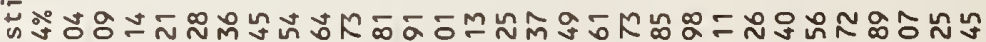

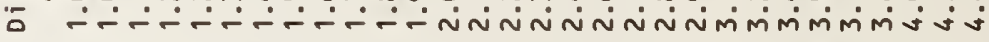

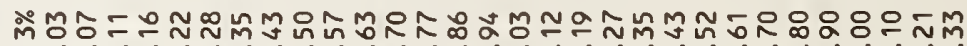
$\because \because \div$ -

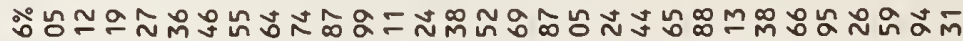

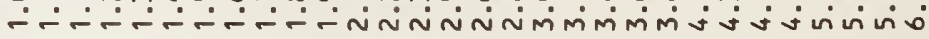
ì

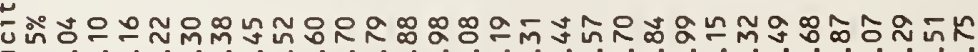

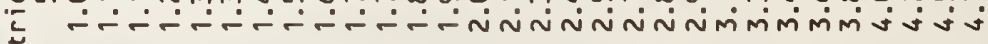

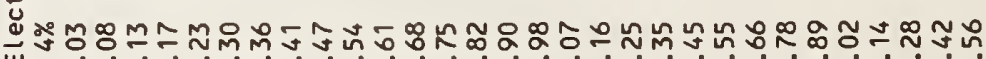
-

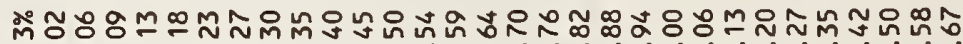

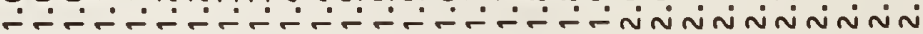

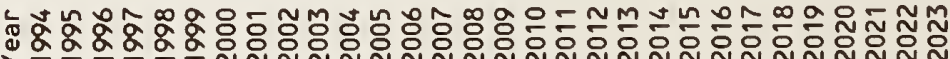




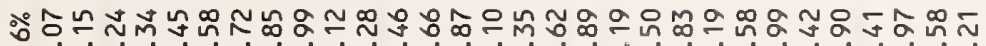

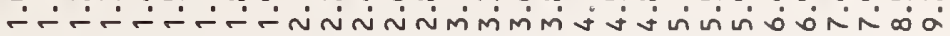

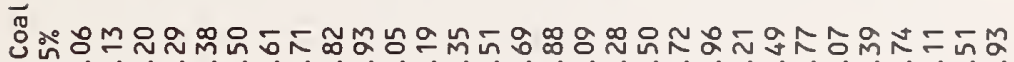

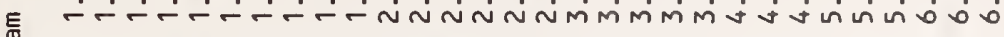

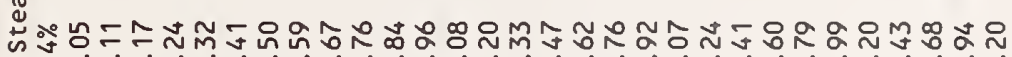

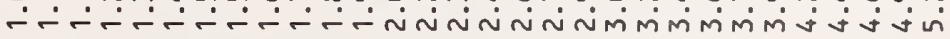

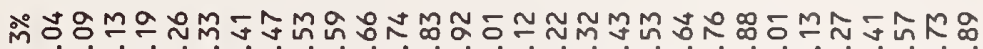

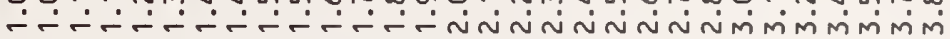

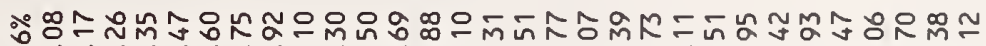

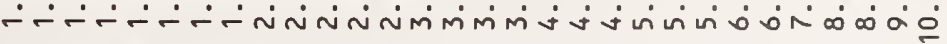
蛋

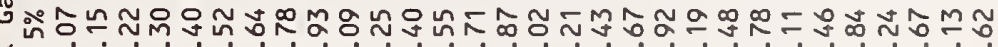

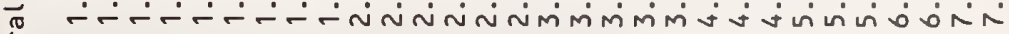

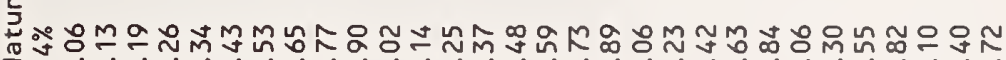

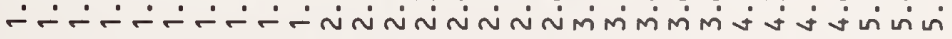

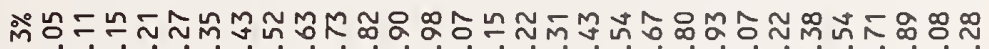

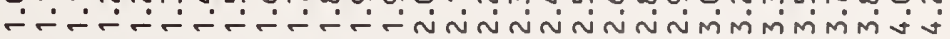

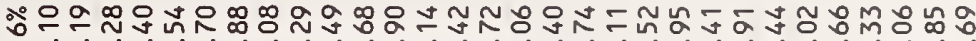
$\overline{0}$ 営

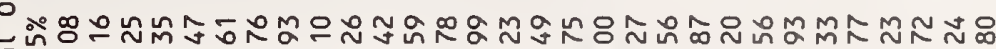

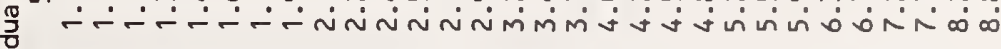

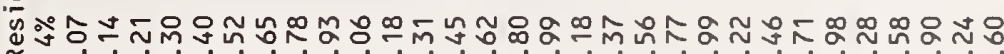

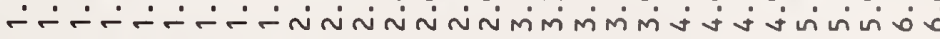

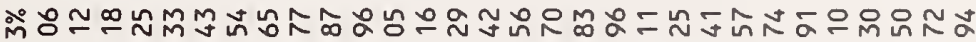

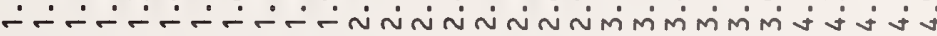

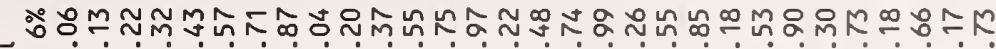

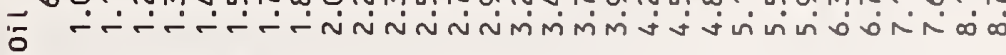

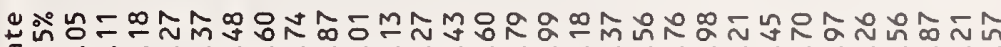

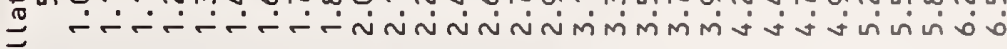
ت

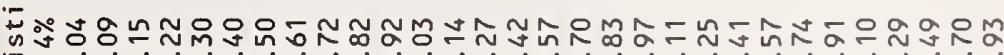

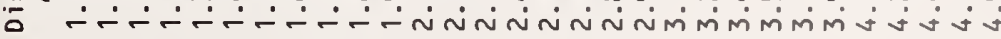

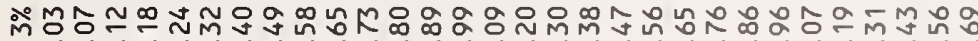

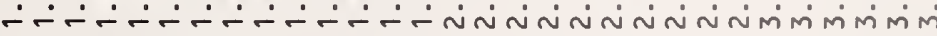

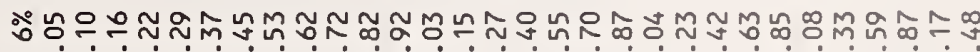

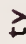

$\because \therefore \therefore \therefore-\because \div$ iñ

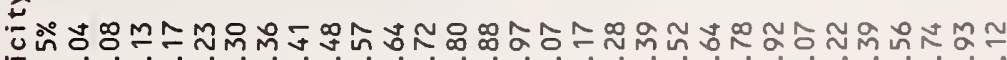

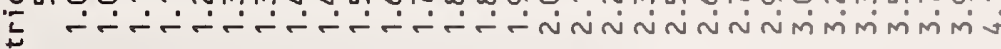

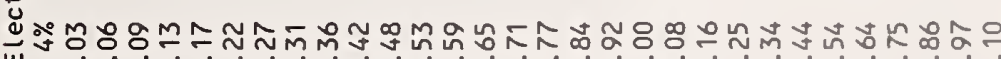

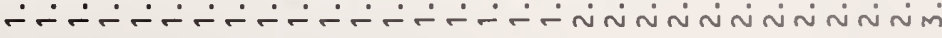

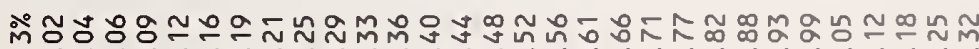

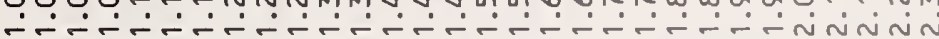

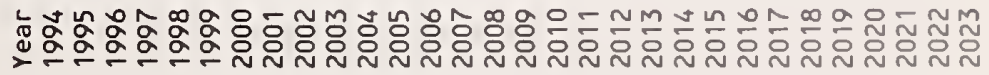




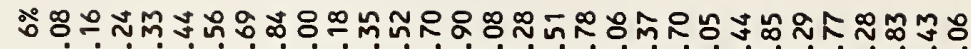
胥

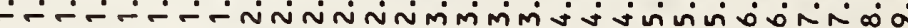

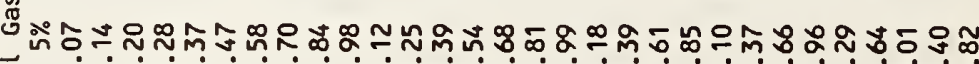

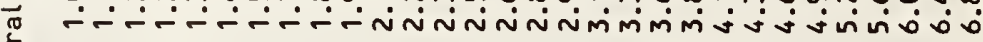

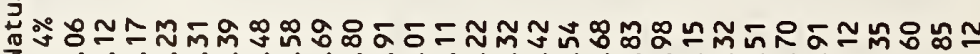

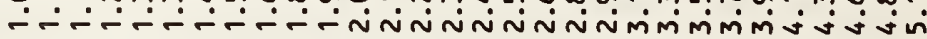

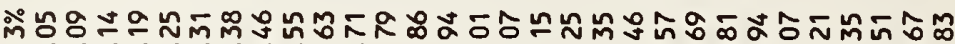

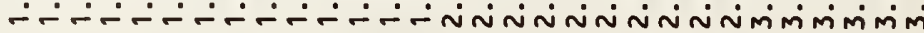

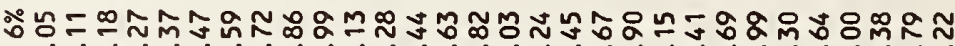

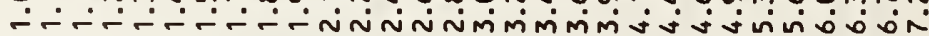

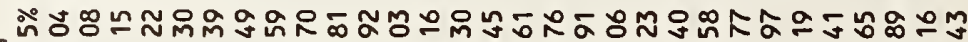

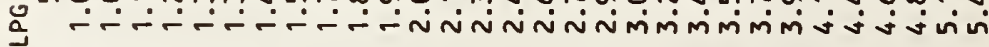
ช゚ฒั $\because \therefore-\therefore$ inininininimimmmmis

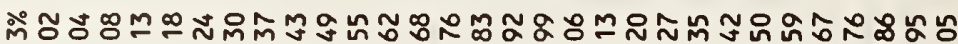
-

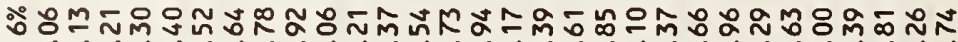
"- $\therefore \therefore-\therefore$ NiñNinimimís

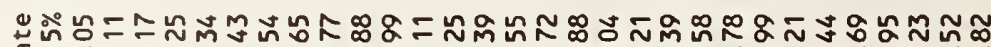
$\stackrel{-\therefore}{=} \div$ N

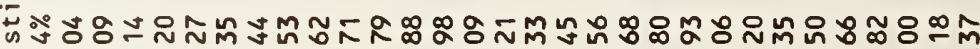
-

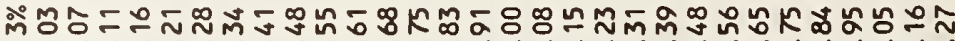

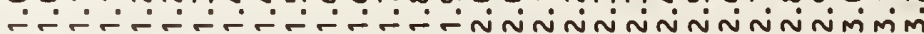

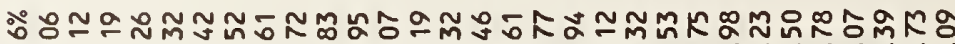
כ

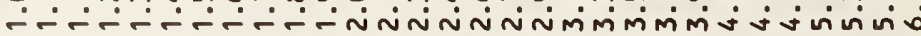

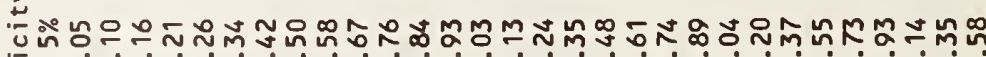

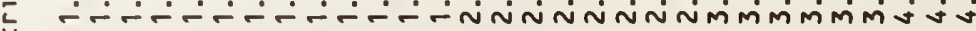

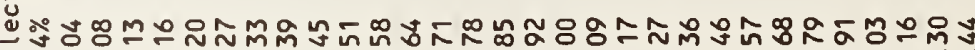

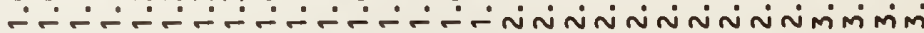

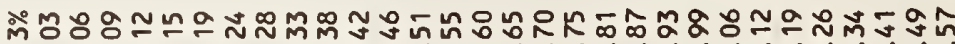

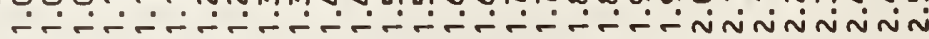

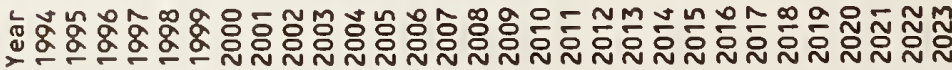




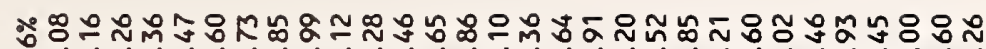

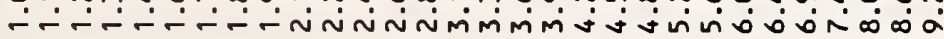

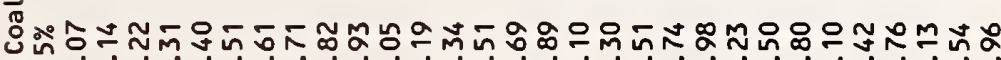

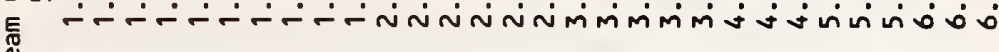

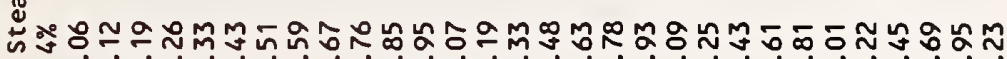
$\therefore \therefore-\div$ iniñ

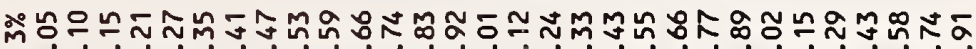

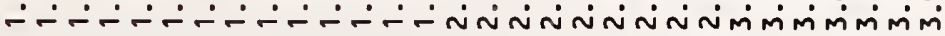

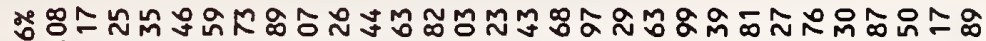
品

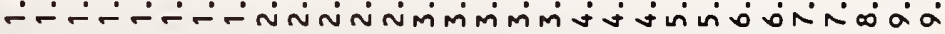

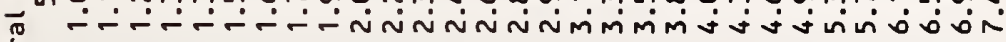

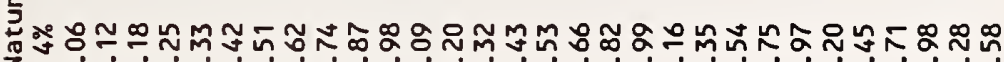

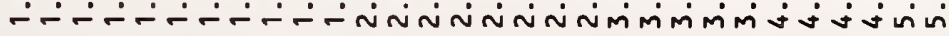

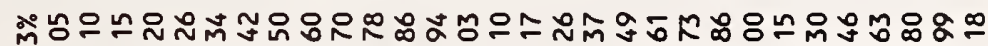
$\because \therefore \therefore-\therefore-\therefore$ iñ $\sim$ ñ

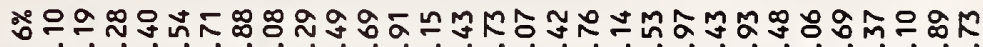
б-

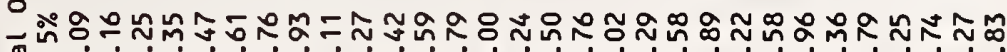

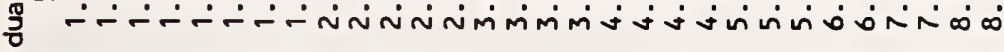
(1)

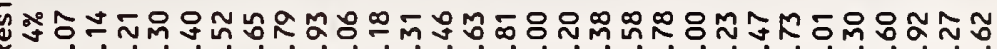

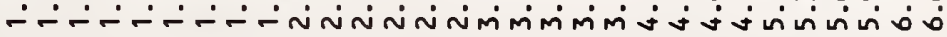

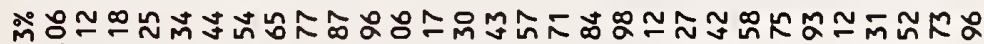
$\therefore \therefore-\therefore-\therefore-\therefore$ iñ

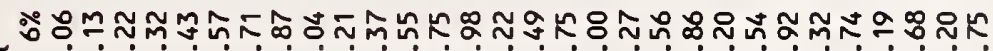

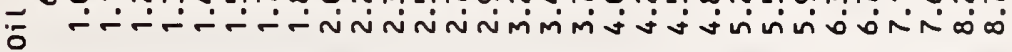

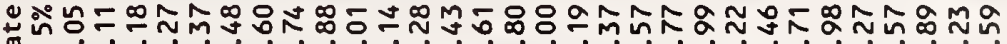
$=$

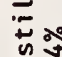

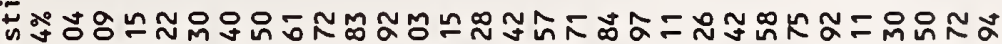

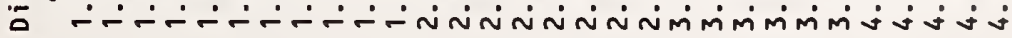

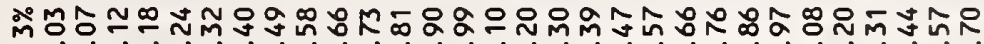

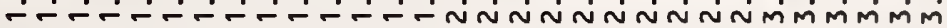

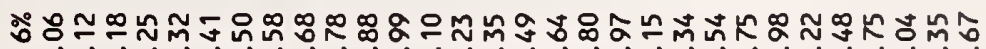
خे $\therefore \therefore \therefore \therefore-\therefore$ iñ

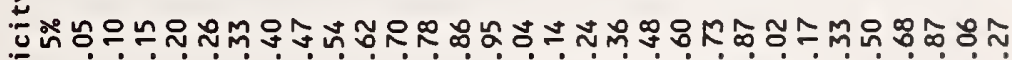

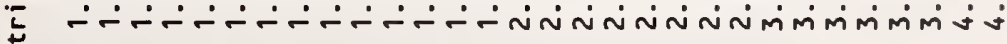

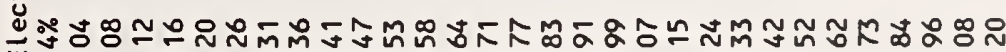

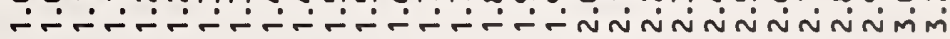

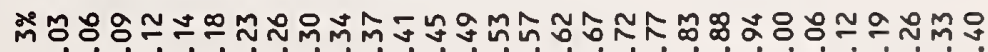

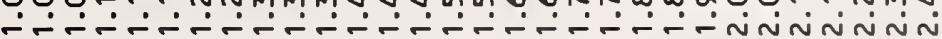

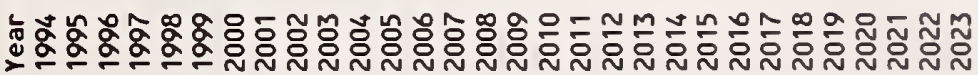




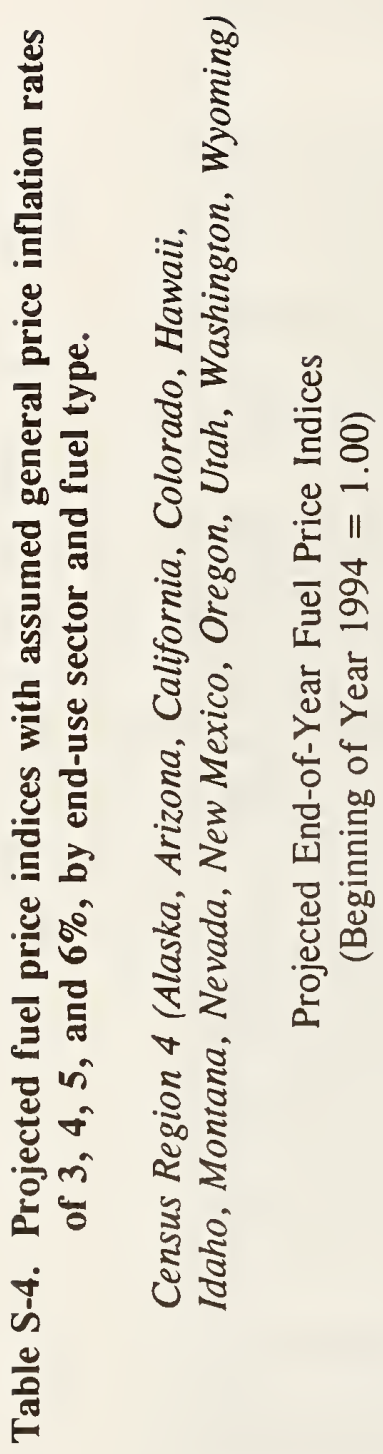

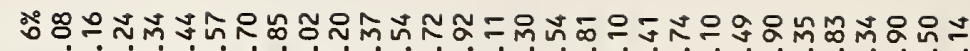

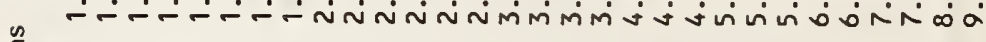
芯 认ำงสกิ

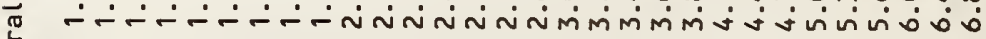

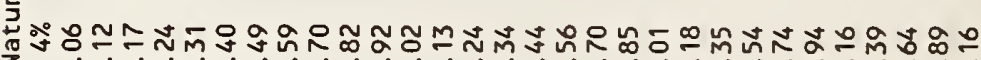

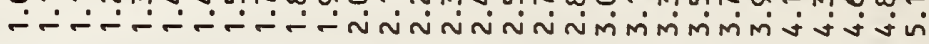

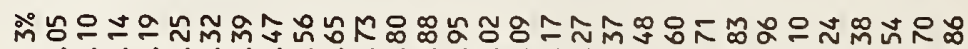

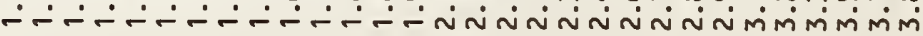

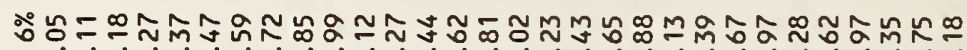

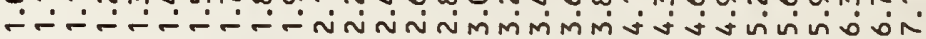

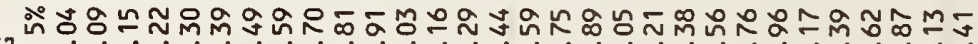
O

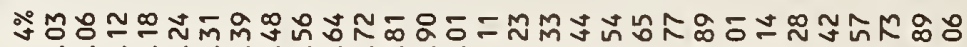
$\therefore \therefore \therefore-\therefore-\therefore$ Niñ

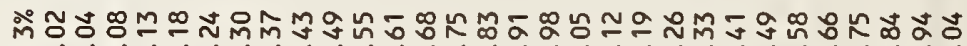

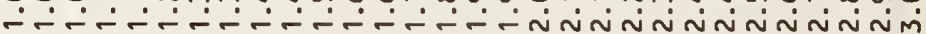

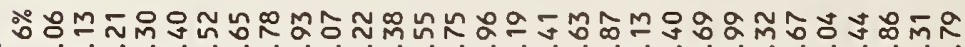

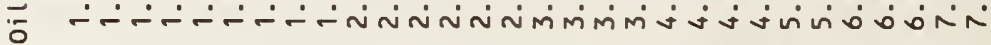

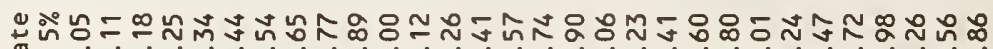
落

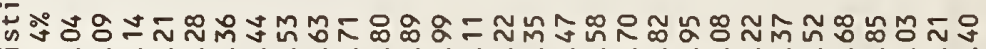

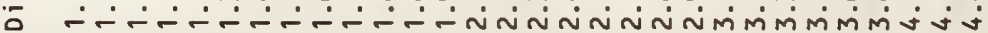

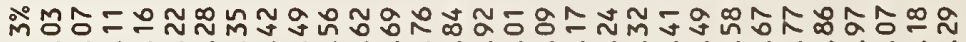

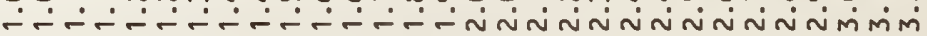

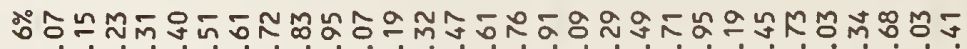
3

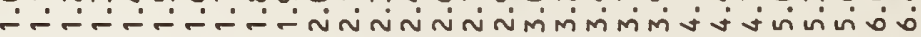

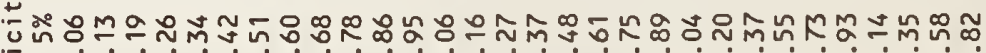

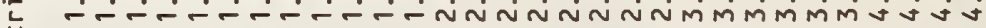

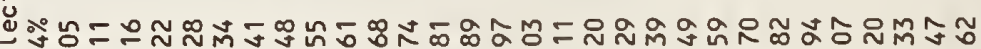

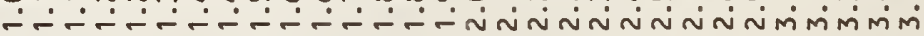

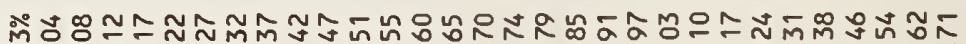

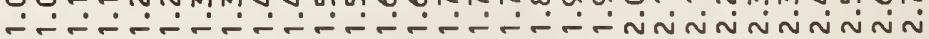

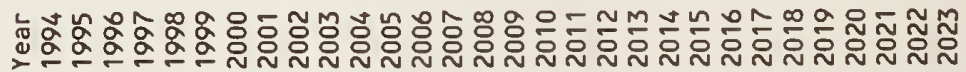




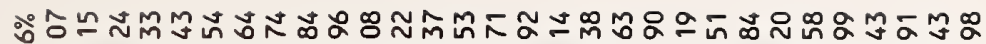
$\because \therefore-\div$ iñ

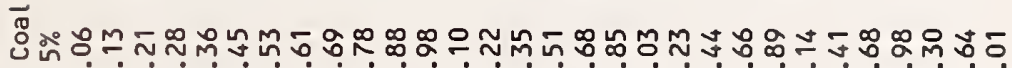

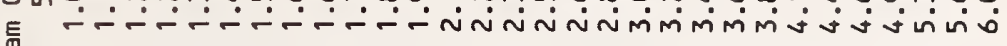
要

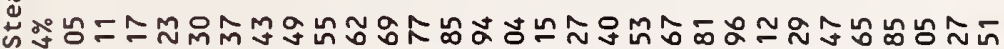

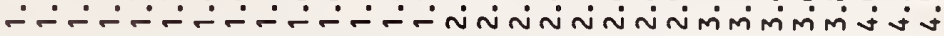

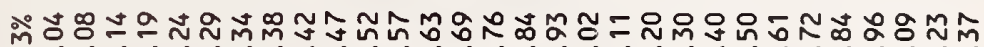

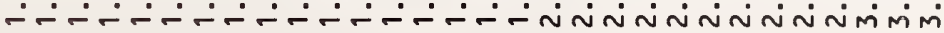

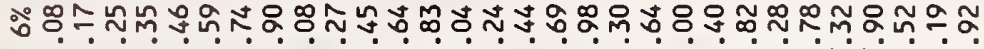
: ภำ

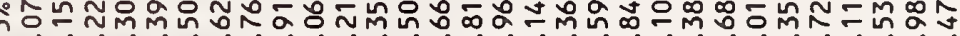

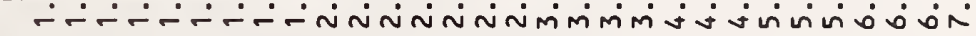

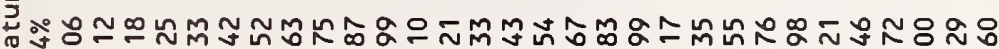
- - - N N Niñ

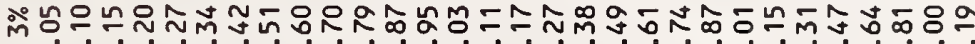

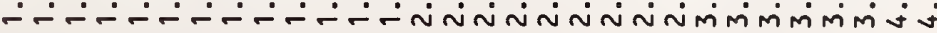

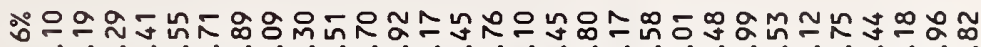

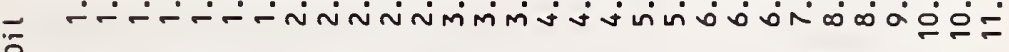

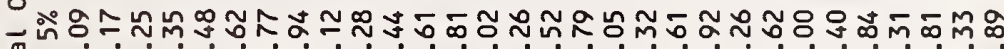

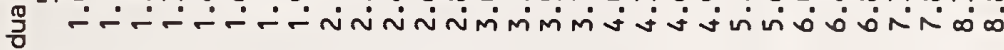

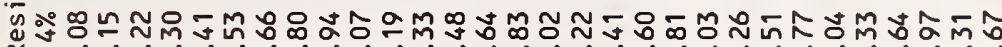

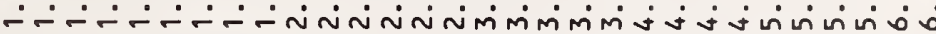

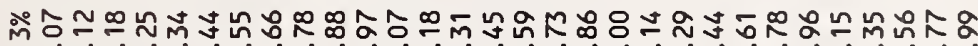

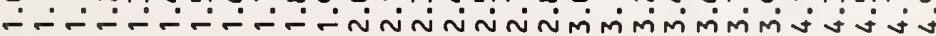

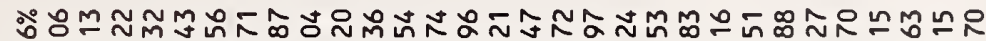

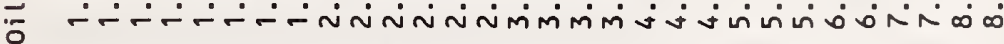

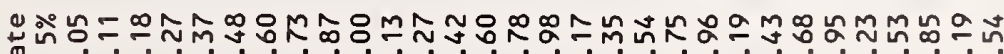

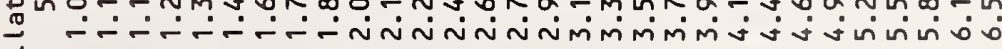

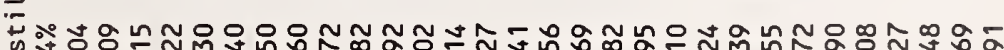

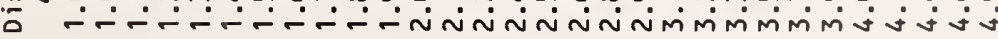

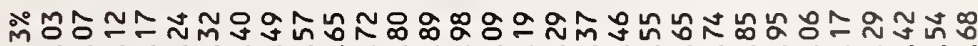

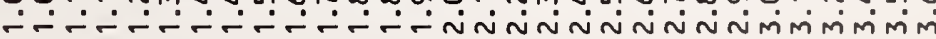

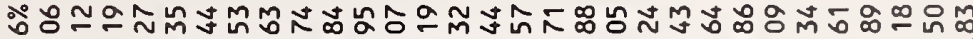
iे

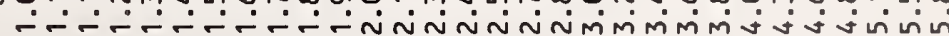
ù i̊ำ 计

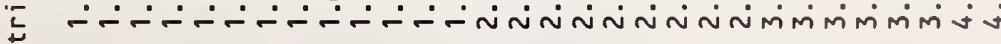

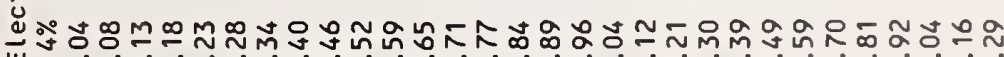
$\because \therefore-\therefore-\therefore$ -

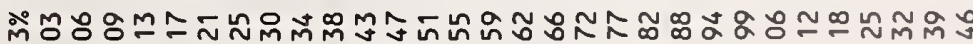

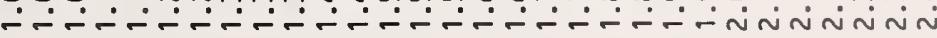

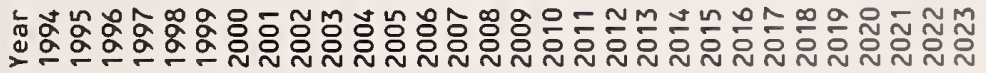




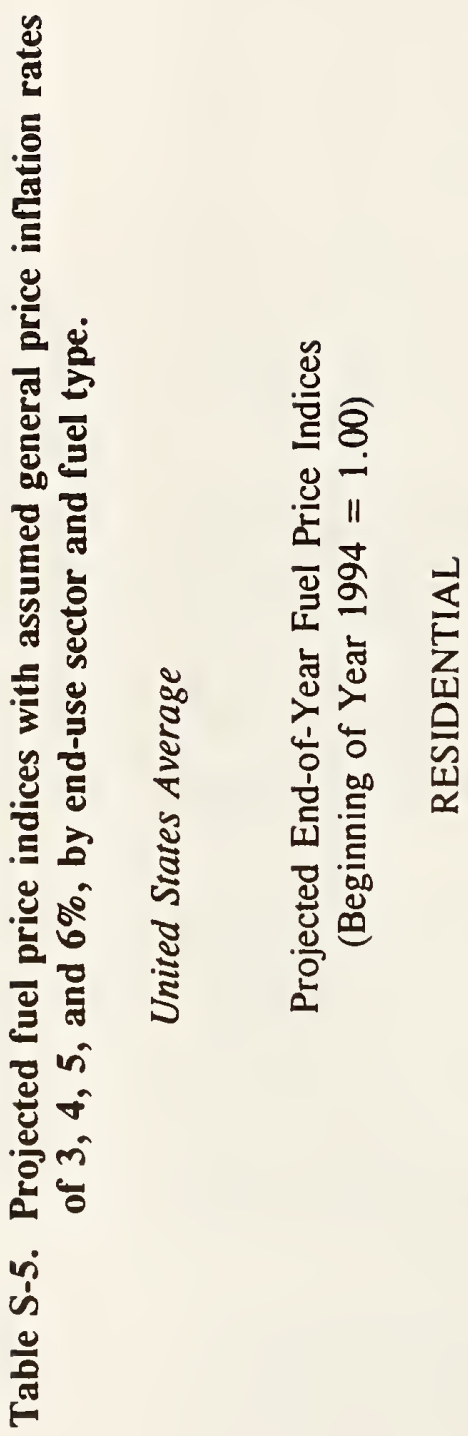

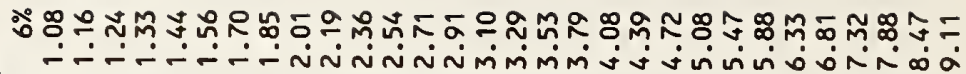
悉

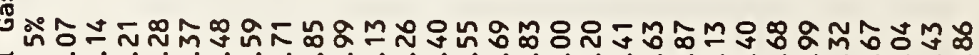

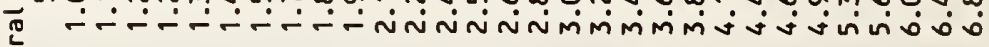

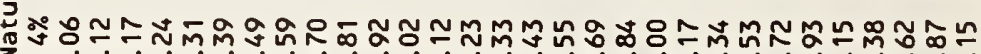

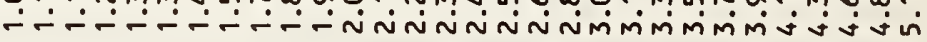

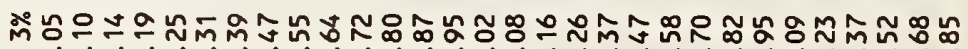

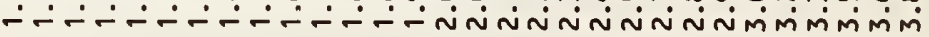

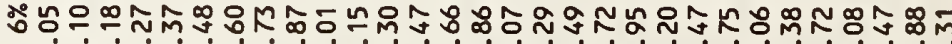

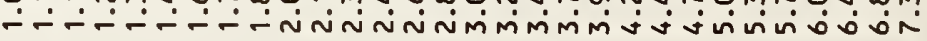

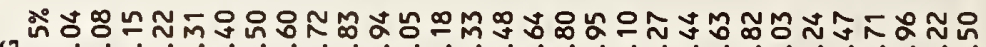
○

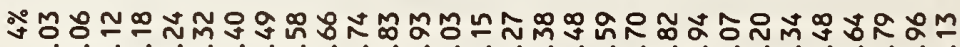
$\because \therefore-\div$ -

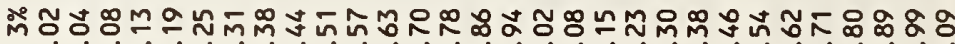

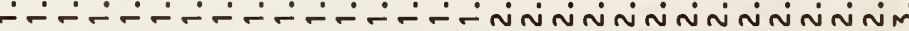

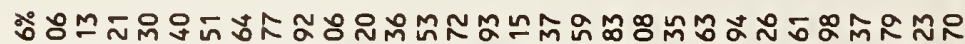
б - - - N NiñNimmimis

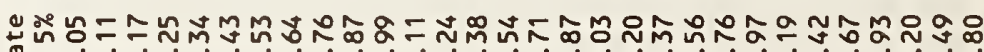
苞すั

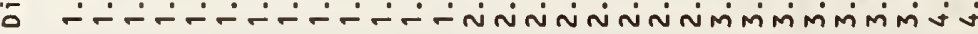

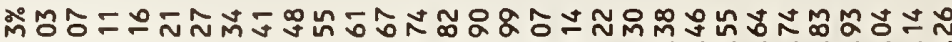

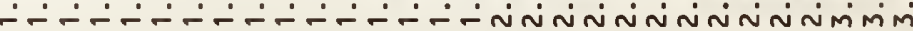

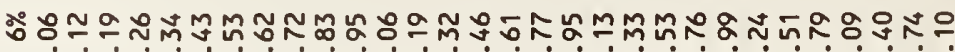

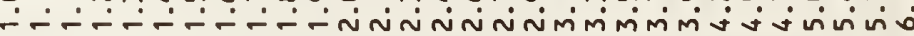
ì

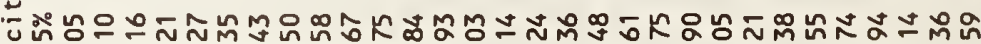
- $-\div$ -

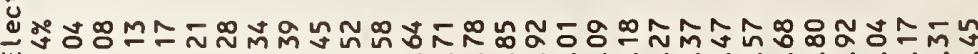

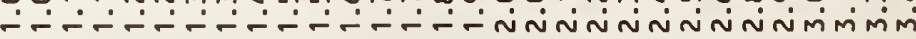

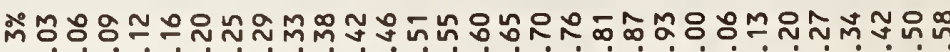

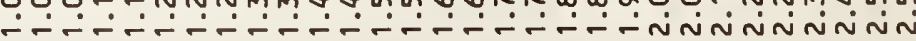

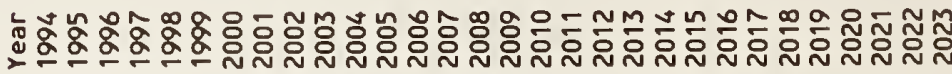




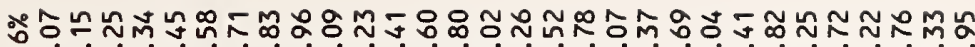
$\because \div-\div$ iñ

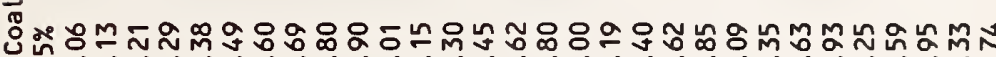

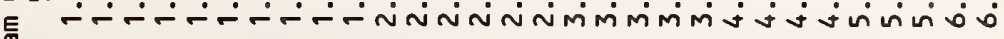

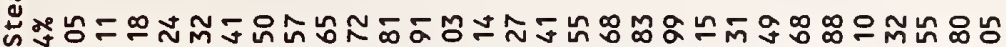

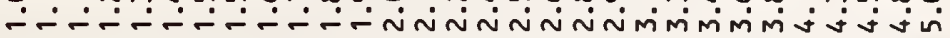

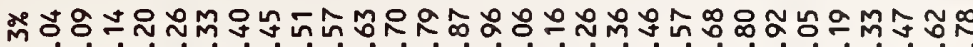

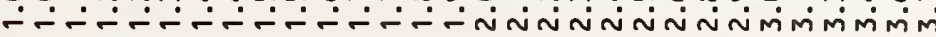

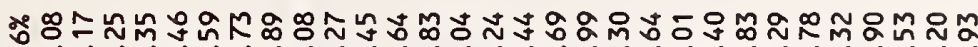

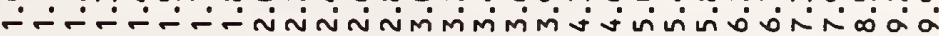

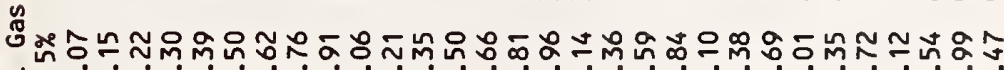

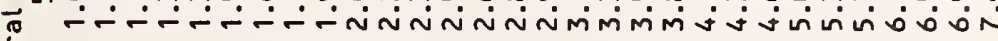
蔍

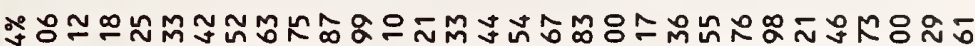

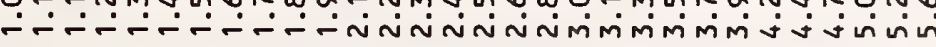

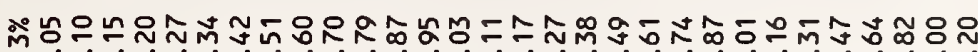

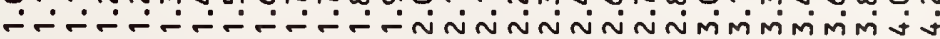

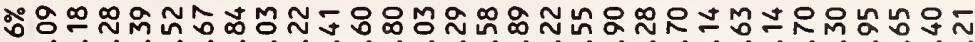

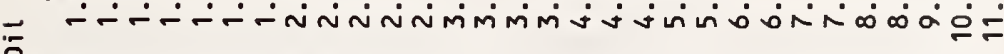
"o

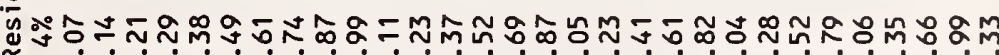

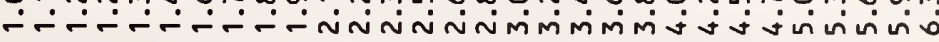

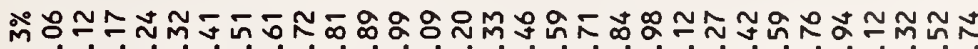
$\because \because \because \because \because 0$ -

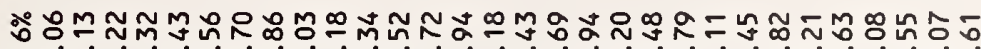

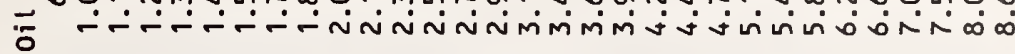

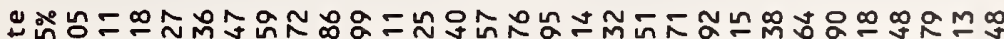

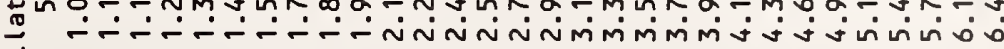

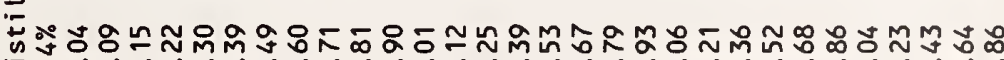
- $\therefore \therefore-\therefore$ N

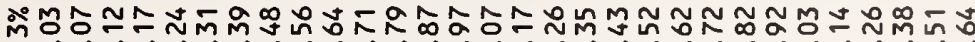
$\because \therefore \therefore \therefore-\therefore-\therefore$ तN

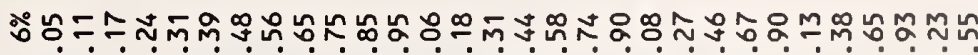
ì

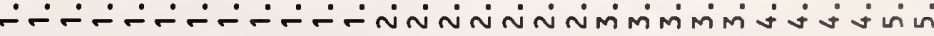

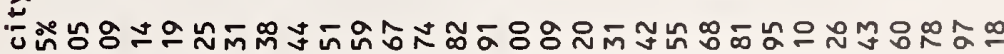

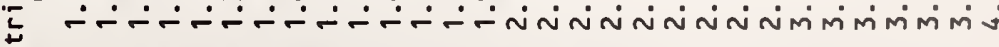

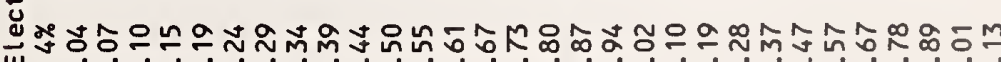

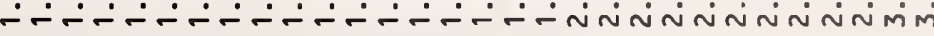

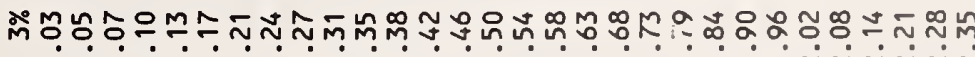

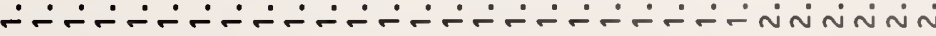

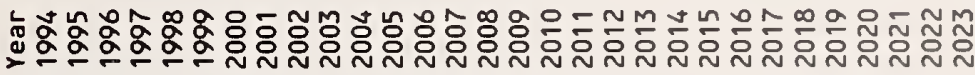




